J. DIFFERENTIAL GEOMETRY

83 (2009) 407-460

\title{
REDUCED GENUS-ONE GROMOV-WITTEN INVARIANTS
}

\author{
AleKsey Zinger
}

\begin{abstract}
In a previous paper, we described a natural closed subset, $\overline{\mathfrak{M}}_{1, k}^{0}(X, A ; J)$, of the moduli space $\overline{\mathfrak{M}}_{1, k}(X, A ; J)$ of stable genusone $J$-holomorphic maps into a symplectic manifold $X$. In this paper we generalize the definition of the main component to moduli spaces of perturbed, in a restricted way, $J$-holomorphic maps and conclude that $\overline{\mathfrak{M}}_{1, k}^{0}(X, A ; J)$, just like $\overline{\mathfrak{M}}_{1, k}(X, A ; J)$, carries a virtual fundamental class, which can be used to define symplectic invariants. These truly genus-one invariants constitute part of the standard genus-one Gromov-Witten invariants, which arise from the entire moduli space $\overline{\mathfrak{M}}_{1, k}(X, A ; J)$. The new invariants are more geometric and can be used to compute the genus-one GW-invariants of complete intersections, as shown in a separate paper.
\end{abstract}

\section{Introduction}

1.1. Background and Motivation. Let $(X, \omega, J)$ be a compact almost Kähler manifold. In other words, $(X, \omega)$ is a symplectic manifold and $J$ is an almost complex structure on $X$ tamed by $\omega$, i.e.

$$
\omega(v, J v)>0 \quad \forall v \in T X-X .
$$

If $g, k$ are nonnegative integers and $A \in H_{2}(X ; \mathbb{Z})$, let $\overline{\mathfrak{M}}_{g, k}(X, A ; J)$ denote the moduli space of (equivalence classes of) stable $J$-holomorphic maps from genus- $g$ Riemann surfaces with $k$ marked points in the homology class $A$. Let

$$
\mathfrak{M}_{g, k}^{0}(X, A ; J) \subset \overline{\mathfrak{M}}_{g, k}(X, A ; J)
$$

be the subspace consisting of the stable maps $[\mathcal{C}, u]$ such that the domain $\mathcal{C}$ is a smooth Riemann surface. The compact moduli space $\overline{\mathfrak{M}}_{g, k}(X, A ; J)$ was constructed in order to "compactify" $\mathfrak{M}_{g, k}^{0}(X, A ; J)$ and to define invariants of $(X, \omega)$ enumerating $J$-holomorphic curves of genus $g$ in $X$. If $g=0,(X, \omega ; A)$ is positive in a certain sense, and $J$ is generic, then $\mathfrak{M}_{g, k}^{0}(X, A ; J)$ is a dense open subset of $\overline{\mathfrak{M}}_{g, k}(X, A ; J)$ and

Partially supported by an NSF Postdoctoral Fellowship.

Received 03/05/2008. 
the corresponding Gromov-Witten invariants do indeed count genuszero $J$-holomorphic curves in $X$; see [McSa, Chapter 7] and [RT, Sections 1,9], for example. However, if $g \geq 1$, it is usually the case that $\mathfrak{M}_{g, k}^{0}(X, A ; J)$ is not dense in $\overline{\mathfrak{M}}_{g, k}(X, A ; J)$ and the genus- $g$ GW-counts include $J$-holomorphic curves of lower genera.

If $g=1$ and $(X, \omega ; A)$ is positive, the above deficiencies are due exclusively to the presence of large subspaces of stable maps $[\mathcal{C}, u]$ in $\overline{\mathfrak{M}}_{1, k}(X, A ; J)$ such that $u$ is constant on the principal components of $\mathcal{C}$, i.e. the irreducible components that carry the genus of $\mathcal{C}$. More precisely, if $m$ is a positive integer, let $\mathfrak{M}_{1, k}^{m}(X, A ; J)$ be the subset of $\overline{\mathfrak{M}}_{1, k}(X, A ; J)$ consisting of the stable maps $[\mathcal{C}, u]$ such that $\mathcal{C}$ is a smooth genus-one curve $\mathcal{C}_{P}$ with $m$ rational components attached directly to $\mathcal{C}_{P},\left.u\right|_{\mathcal{C}_{P}}$ is constant, and the restriction of $u$ to each rational component is non-constant. Figure 1 shows the domain of an element of $\mathfrak{M}_{1, k}^{3}(X, A ; J)$, from the points of view of symplectic topology and of algebraic geometry. In the first diagram, each shaded disc represents a sphere; the homology class next to each rational component $\mathcal{C}_{i}$ indicates the degree of $\left.u\right|_{\mathcal{C}_{i}}$. In the second diagram, the components of $\mathcal{C}$ are represented by curves, and the pair of indices next to each component $\mathcal{C}_{i}$ shows the genus of $\mathcal{C}_{i}$ and the degree of $\left.u\right|_{\mathcal{C}_{i}}$. We denote by $\overline{\mathfrak{M}}_{1, k}^{m}(X, A ; J)$ the closure of $\mathfrak{M}_{1, k}^{m}(X, A ; J)$ in $\overline{\mathfrak{M}}_{1, k}(X, A ; J)$. The image $u(\mathcal{C})$ of an element of $\overline{\mathfrak{M}}_{1, k}^{m}(X, A ; J)$ is a genus-zero, instead of genusone, $J$-holomorphic curve in $X$. We note that if $J$ is sufficiently regular, then

$$
\begin{aligned}
\operatorname{dim} \mathfrak{M}_{1, k}^{0}(X, A ; J) & =2\left(\left\langle c_{1}(T X), A\right\rangle+k\right) \equiv \operatorname{dim}_{1, k}(X, A) \quad \text { and } \\
\operatorname{dim} \mathfrak{M}_{1, k}^{m}(X, A ; J) & =\operatorname{dim}_{1, k}(X, A)+2(n-m),
\end{aligned}
$$

where $2 n$ is the real dimension of $X$. It follows that the complement of $\mathfrak{M}_{1, k}^{0}(X, A ; J)$ in $\overline{\mathfrak{M}}_{1, k}(X, A ; J)$ contains subspaces of dimension at least as large as the dimension of $\mathfrak{M}_{1, k}^{0}(X, A ; J)$, as long as $n \geq 1$, i.e. $X$ is not a finite collection of points.

Definition 1.1 in [Z4] describes a subset $\overline{\mathfrak{M}}_{1, k}^{0}(X, A ; J)$ of $\overline{\mathfrak{M}}_{1, k}(X, A ; J)$, for an arbitrary compact almost Kähler manifold $(X, \omega, J)$; this subset is obtained from $\overline{\mathfrak{M}}_{1, k}(X, A ; J)$ by discarding most elements of the spaces $\overline{\mathfrak{M}}_{1, k}^{m}(X, A ; J)$ with $m \leq n$. In particular, $\overline{\mathfrak{M}}_{1, k}^{0}(X, A ; J)$ contains $\mathfrak{M}_{1, k}^{0}(X, A ; J)$. By $\left[\mathbf{Z 4}\right.$, Theorem 1.2], $\overline{\mathfrak{M}}_{1, k}^{0}(X, A ; J)$ is a closed subset of $\overline{\mathfrak{M}}_{1, k}(X, A ; J)$ and thus is compact. If $(X, \omega ; A)$ is positive in the same sense as in the genus-zero case and $J$ is generic, $\mathfrak{M}_{1, k}^{0}(X, A ; J)$ is a dense open subset of $\overline{\mathfrak{M}}_{1, k}^{0}(X, A ; J)$. In addition, $\overline{\mathfrak{M}}_{1, k}^{0}(X, A ; J)$ 

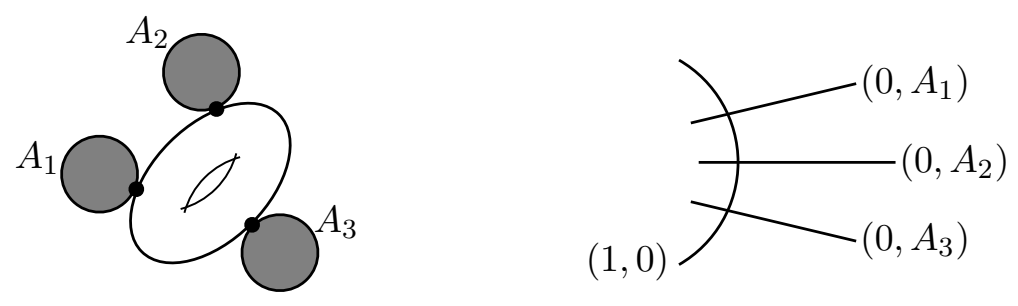

$$
A_{1}+A_{2}+A_{3}=A, \quad A_{1}, A_{2}, A_{3} \neq 0
$$

Figure 1. The domain of an element of $\mathfrak{M}_{1, k}^{3}(X, A ; J)$.

carries a rational fundamental class, which can be used to define a symplectic invariant of $(X, \omega)$ counting genus-one $J$-holomorphic curves in $X$, without any genus-zero contribution in contrast to the standard Gromov-Witten invariants; see [Z4, Subsection 1.3]. Unlike the genuszero case, $\overline{\mathfrak{M}}_{1, k}^{0}(X, A ; J)$ has the topological structure of a singular, instead of smooth, orbivariety.

A $J$-holomorphic map into $X$ is a smooth map $u$ from a Riemann surface $(\Sigma, j)$ that satisfies the Cauchy-Riemann equation corresponding to $(J, j)$ :

$$
\bar{\partial}_{J, j} u \equiv \frac{1}{2}(d u+J \circ d u \circ j)=0 .
$$

The Riemann surface $(\Sigma, j)$ may have simple nodes. In this paper we generalize the results of $[\mathbf{Z 4}]$ to $L_{1}^{p}$-maps $u$, from genus-one Riemann surfaces, that satisfy a family of perturbed Cauchy-Riemann equations:

$$
\bar{\partial}_{J, j} u+\nu(u)=0 \text {. }
$$

The perturbation term $\nu(u)$ is a section of the vector bundle

$$
\Lambda_{J, j}^{0,1} T^{*} \Sigma \otimes u^{*} T X \equiv\left\{\eta \in \operatorname{Hom}_{\mathbb{R}}\left(T \Sigma, u^{*} T X\right): J \circ \eta=-\eta \circ j\right\} \longrightarrow \Sigma .
$$

We will study the moduli space $\overline{\mathfrak{M}}_{1, k}(X, A ; J, \nu)$ of $(J, \nu)$-holomorphic maps, i.e. of solutions to the perturbed Cauchy-Riemann equations, for a continuous family $\nu=\nu(u)$ chosen from a proper linear subspace of the space of all such families; see Definition 1.2. A key condition on $\nu$ will be that if the degree of $u$ restricted to the principal components of $\Sigma$ is zero, then the restriction of $\nu(u)$ to the principal components and all nearby degree-zero bubble components is also zero. Such a family $\nu=\nu(u)$ will be called effectively supported.

We will show that if $\nu$ is sufficiently small and effectively supported, then the moduli space $\overline{\mathfrak{M}}_{1, k}(X, A ; J, \nu)$ contains a natural closed subspace $\overline{\mathfrak{M}}_{1, k}^{0}(X, A ; J, \nu)$ containing $\mathfrak{M}_{1, k}^{0}(X, A ; J, \nu)$, i.e. the subspace of maps with smooth domains; see Definition 1.3 and Theorem 1.4. For a 
generic choice of $\nu$, the "boundary" of $\overline{\mathfrak{M}}_{1, k}^{0}(X, A ; J, \nu)$ is of real codimension two and thus $\overline{\mathfrak{M}}_{1, k}^{0}(X, A ; J, \nu)$ determines a rational homology class. This virtual fundamental class (VFC) for $\overline{\mathfrak{M}}_{1, k}^{0}(X, A ; J)$ does not change under small changes in $\nu$ and is an invariant of $(X, \omega)$. It can be used to define new GW-style invariants, which we denote by $\mathrm{GW}_{1, k}^{0}$. These invariants differ from the standard GW-invariants by a combination of the genus-zero GW-invariants of $X$; see Subsection 1.2 below for some special cases.

We note that effectively supported families $\nu=\nu(u)$ are in no sense generic in the space of all families. If fact, for a generic $\nu, \mathfrak{M}_{1, k}^{0}(X, A ; J$, $\nu)$ is dense in $\overline{\mathfrak{M}}_{1, k}(X, A ; J, \nu)$, and the latter space determines the standard $\mathrm{GW}$-invariants of $(X, \omega)$. In particular, the statements of the previous paragraph do not hold for a generic family $\nu$ of perturbations.

An algebraic approach to reduced genus-one GW-invariants is suggested by Vakil and the author at the end of [VaZ2]. It still remains to verify that the resulting algebraic invariants agree with the symplectic ones defined in this paper (whenever the target space is a smooth algebraic variety), but this should be deducible from the desingularizations for certain natural sheaves constructed by Vakil and the author in [VaZ1, Section 5].

Since the symplectic invariants arising from the smaller moduli space $\overline{\mathfrak{M}}_{1, k}^{0}(X, A ; J, \nu)$, with $\nu$ effectively supported, are closely related to the standard GW-invariants, they do not in principle carry any new information. In practice, they behave better geometrically. In particular, Li and the author show in $[\mathbf{L Z}]$ that there is a simple relation between reduced genus-one GW-invariants of a projective complete intersection and twisted reduced genus-one GW-invariants of the ambient space. This relation mimics the corresponding well-known relation in genus zero (see [LZ, (1.2)], for example), but no relation in positive genera had been even conjectured until $[\mathbf{L Z}]$. Combining $[\mathbf{L Z}$, Theorem 1.1] for the reduced genus-one invariants constructed in this paper with the desingularization of [VaZ1] and Theorem 1.1 below, the author confirms the 1993 mirror symmetry of Bershadsky-Cecotti-Ooguri-Vafa [BCOV] for the genus-one GW-invariants of a quintic threefold in $[\mathbf{Z 5}]$; this is the genus-one analogue of the 1991 genus-zero mirror symmetry prediction of Candelas-de la Ossa-Green-Parkes [CDGP], which was proved in several different ways in the mid to late 90 s.

In Subsection 1.3, we recall some aspects of the VFC constructions of Fukaya-Ono $[\mathbf{F u O n}]$ and Li-Tian $[\mathbf{L T}]$ which are well suited for defining a VFC for $\overline{\mathfrak{M}}_{1, k}^{0}(X, A ; J)$. We state the main results of this paper 
in Subsection 1.4. In Subsections 2.1 and 2.2, we generalize the setup of $[\mathbf{Z 4}]$ for $J$-holomorphic maps to $(J, \nu)$-holomorphic maps. In Subsection 2.3, we state three propositions that together are equivalent to Theorem 1.4. They are proved in Subsections 2.4 and 2.5 by extending some of the analytic arguments of $[\mathbf{Z 4}$, Sections 3,4] to the present situation. The difference between the standard and reduced GW-invariants is analyzed in Section 3; see also the next subsection.

1.2. Standard vs. Reduced Gromov-Witten Invariants. From the construction of VFC for $\overline{\mathfrak{M}}_{1, k}^{0}(X, A ; J)$ in Subsection 1.4, it is immediate that the difference between the standard and reduced genus-one GW-invariants of $X$ must be a combination of the genus-zero GWinvariants of $X$. The exact form of this combination can be determined in each specific case from Proposition 3.1. In this subsection, we give an explicit expression for the difference between the standard and reduced genus-one GW-invariants in the two simplest cases.

For each $l=1, \ldots, k$, let

$$
\mathrm{ev}_{l}: \overline{\mathfrak{M}}_{g, k}(X, A ; J) \longrightarrow X, \quad\left[\Sigma, y_{1}, \ldots, y_{k} ; u\right] \longrightarrow u\left(y_{l}\right),
$$

the evaluation map at the $l$-th marked point. We will call a cohomology class $\psi$ on $\overline{\mathfrak{M}}_{g, k}(X, A ; J)$ geometric if $\psi$ is a product of the classes $e_{l}^{*} \mu_{l}$ for $\mu_{l} \in H^{*}(X ; \mathbb{Z})$. We denote by $\overline{\mathbb{Z}}^{+}$the set of nonnegative integers.

Theorem 1.1. Suppose $(X, \omega)$ is a compact symplectic manifold, $A \in H_{2}(X ; \mathbb{Z})^{*}, k \in \overline{\mathbb{Z}}^{+}$. If $J$ is an $\omega$-compatible almost complex structure on $X$ and $\psi$ is a geometric cohomology class on $\overline{\mathfrak{M}}_{1, k}(X, A ; J)$, then

$$
\begin{aligned}
\mathrm{GW}_{1, k}^{X}(A ; \psi)- & \mathrm{GW}_{1, k}^{0 ; X}(A ; \psi) \\
= & \begin{cases}0, & \text { if } \operatorname{dim}_{\mathbb{R}} X=4 ; \\
\frac{2-\left\langle c_{1}(T X), A\right\rangle}{24} \mathrm{GW}_{0, k}^{X}(A ; \psi), & \text { if } \operatorname{dim}_{\mathbb{R}} X=6 .\end{cases}
\end{aligned}
$$

Theorem 1.1 is proved in Section 3 by studying the obstruction theory along each stratum of the moduli space $\overline{\mathfrak{M}}_{1, k}(X, A ; J, \nu)$, after capping it with $\psi$. This proof generalizes to higher-dimensional manifolds $X$ and more general cohomology classes; an explicit formula is obtained in [Z6]. In fact, for geometric cohomology classes and higher-dimensional manifolds $X$, the difference is given by an expression similar to the correction term in [Z3, Theorem 1.1]; this can be seen a priori from Proposition 3.1 and [Z3, Subsection 3.2]. A special case of Theorem 1.1 is [Ge, Theorem A]; its proof has not yet appeared.

Theorem 1.1 has a natural, but rather speculative, generalization to higher-genus invariants. Suppose that the main component

$$
\overline{\mathfrak{M}}_{g, k}^{0}(X, A ; J) \subset \overline{\mathfrak{M}}_{g, k}(X, A ; J)
$$


is well-defined, as is the main component

$$
\overline{\mathfrak{M}}_{g, k}^{0}(X, A ; J, \nu) \subset \overline{\mathfrak{M}}_{g, k}(X, A ; J, \nu)
$$

for a sufficiently large subspace of perturbations $\nu$ of the $\bar{\partial}_{J}$-operator so that $\overline{\mathfrak{M}}_{g, k}(X, A ; J, \nu)$ has a regular structure for a generic $\nu$ in this subspace; see Subsection 1.4 for the $g=1$ case. If so, $\overline{\mathfrak{M}}_{g, k}^{0}(X, A ; J)$ carries a virtual fundamental class and determines reduced genus- $g$ GWinvariants $\mathrm{GW}_{g, k}^{0 ; X}(A ; \psi)$. Theorem 1.1 and its proof should then generalize to higher-genus invariants. If $\operatorname{dim}_{\mathbb{R}} X=6$, the expected relationship is

$$
\mathrm{GW}_{g, k}^{X}(A ; \psi)-\mathrm{GW}_{g, k}^{0 ; X}(A ; \psi)=\sum_{g^{\prime}=0}^{g-1} C_{g}^{g^{\prime}}\left(\left\langle c_{1}(T X), A\right\rangle\right) \mathrm{GW}_{g^{\prime}, k}^{0 ; X}(A ; \psi),
$$

where $\mathrm{GW}_{0, k}^{0} \equiv \mathrm{GW}_{0, k}$. The coefficients $C_{g}^{g^{\prime}}\left(\left\langle c_{1}(T X), A\right\rangle\right)$ are given by Hodge integrals, i.e. integrals of natural cohomology classes on the moduli spaces $\overline{\mathcal{M}}_{*, *}$ of curves. They are of the form expected from the usual obstruction bundle approach. For example,

$$
\begin{gathered}
C_{2}^{1}((5-a) d)=-\frac{d(a-5)}{24}, \\
C_{2}^{0}((5-a) d)=\frac{1}{2}\left(\frac{2+d(a-5)}{24}\right)^{2} \\
+\left\langle c\left(\mathbb{E}^{*} \otimes T X\right) c\left(L_{2,1} \otimes T \mathbb{P}^{1}\right)^{-1},\left[\overline{\mathcal{M}}_{2,1}\right] \times\left[\mathbb{P}^{1}\right]\right\rangle,
\end{gathered}
$$

where $L_{2,1} \longrightarrow \overline{\mathcal{M}}_{2,1}$ is the universal tangent line bundle, $\mathbb{E} \longrightarrow \overline{\mathcal{M}}_{2,1}$ is the rank-two Hodge bundle, and $\mathbb{P}^{1}$ is viewed as a smooth degree- $d$ curve in $Y$. The coefficients $C_{g}^{g^{\prime}}\left(\left\langle c_{1}(T X), A\right\rangle\right)$ can be expressed in terms of the numbers $C_{g^{\prime}}\left(g-g^{\prime}, X, A\right)$ of $[\mathbf{P a}]$ and vice versa.

1.3. Configuration Spaces. In this subsection we recall certain configuration spaces that are standard in the theory of Gromov-Witten invariants. We then define what we mean by effectively supported perturbations of the $\bar{\partial}_{J}$-operator that are central to this paper.

Fix $p>2$. Suppose $X$ is a compact manifold, $A \in H_{2}(X ; \mathbb{Z})$, and $g, k \in$ $\overline{\mathbb{Z}}^{+}$. We denote by $\mathfrak{X}_{g, k}(X, A)$ the space of equivalence classes of stable $L_{1}^{p}$-maps $u: \Sigma \longrightarrow X$ from genus- $g$ Riemann surfaces with $k$ marked points, which may have simple nodes, to $X$ of degree $A$, i.e.

$$
u_{*}[\Sigma]=A \in H_{2}(X ; \mathbb{Z}) .
$$

Let $\mathfrak{X}_{g, k}^{0}(X, A)$ be the subset of $\mathfrak{X}_{g, k}(X, A)$ consisting of the stable maps with smooth domains. The spaces $\mathfrak{X}_{g, k}(X, A)$ are topologized using $L_{1}^{p}$ convergence on compact subsets of smooth points of the domain and certain convergence requirements near the nodes; see [LT, Section 3]. 
The spaces $\mathfrak{X}_{g, k}(X, A)$ can be stratified by infinite-dimensional orbifolds $\mathfrak{X}_{\mathcal{T}}(X)$ of stable maps from domains of the same geometric type and with the same degree distribution between the components of the domain; each stratum is a quotient of a smooth Banach manifold $\tilde{\mathfrak{X}}_{\mathcal{T}}(X)$ by a finite-dimensional Lie group $G_{\mathcal{T}}$. The closure of the main stratum, $\mathfrak{X}_{g, k}^{0}(X, A)$, is $\mathfrak{X}_{g, k}(X, A)$.

If $J$ is an almost complex structure on $X$, let

$$
\Gamma_{g, k}^{0,1}(X, A ; J) \longrightarrow \mathfrak{X}_{g, k}(X, A)
$$

be the bundle of $(T X, J)$-valued $(0,1) L^{p}$-forms. In other words, the fiber of $\Gamma_{g, k}^{0,1}(X, A ; J)$ over a point $[b]=[\Sigma, j ; u]$ in $\mathfrak{X}_{g, k}(X, A)$ is the space

$$
\begin{gathered}
\left.\Gamma_{g, k}^{0,1}(X, A ; J)\right|_{[b]}=\Gamma^{0,1}(b ; J) / \operatorname{Aut}(b), \quad \text { where } \\
\Gamma^{0,1}(b ; J)=L^{p}\left(\Sigma ; \Lambda_{J, j}^{0,1} T^{*} \Sigma \otimes u^{*} T X\right) .
\end{gathered}
$$

Here $j$ is the complex structure on $\Sigma$, the domain of the smooth map $u$. The bundle $\Lambda_{J, j}^{0,1} T^{*} \Sigma \otimes u^{*} T X$ over $\Sigma$ consists of $(J, j)$-antilinear homomorphisms:

$$
\Lambda_{J, j}^{0,1} T^{*} \Sigma \otimes u^{*} T X=\left\{\eta \in \operatorname{Hom}\left(T \Sigma, u^{*} T X\right): J \circ \eta=-\eta \circ j\right\} .
$$

The total space of the bundle $\Gamma_{g, k}^{0,1}(X, A ; J) \longrightarrow \mathfrak{X}_{g, k}(X, A)$ is topologized using $L^{p}$-convergence on compact subsets of smooth points of the domain and certain convergence requirements near the nodes. The restriction of $\Gamma_{g, k}^{0,1}(X, A ; J)$ to each stratum $\mathfrak{X}_{\mathcal{T}}(X)$ is a quotient of a smooth Banach vector bundle $\tilde{\Gamma}_{\mathcal{T}}^{0,1}(X ; J)$ over $\tilde{\mathfrak{X}}_{\mathcal{T}}(X)$ by $G_{\mathcal{T}}$. The smooth sections of the bundles $\tilde{\Gamma}_{\mathcal{T}}^{0,1}(X ; J) \longrightarrow \tilde{\mathfrak{X}}_{\mathcal{T}}(X)$ given by

$$
\bar{\partial}_{J}([\Sigma, j ; u])=\bar{\partial}_{J, j} u=\frac{1}{2}(d u+J \circ d u \circ j)
$$

induce sections of $\Gamma_{g, k}^{0,1}(X, A ; J)$ over $\mathfrak{X}_{\mathcal{T}}(X)$, which define a continuous section $\bar{\partial}_{J}$ of the bundle

$$
\Gamma_{g, k}^{0,1}(X, A ; J) \longrightarrow \mathfrak{X}_{g, k}(X, A) .
$$

The zero set of this section is the moduli space $\overline{\mathfrak{M}}_{g, k}(X, A ; J)$ of equivalence classes of stable $J$-holomorphic degree- $A$ maps from genus- $g$ curves with $k$ marked points into $X$. The section $\bar{\partial}_{J}$ over $\tilde{\mathfrak{X}}_{\mathcal{T}}(X)$ is Fredholm, i.e. its linearization has finite-dimensional kernel and cokernel at every point of the zero set. The index of the linearization of $\bar{\partial}_{J}$ at an element of $\mathfrak{M}_{g, k}^{0}(X, A ; J)$ is the expected dimension $\operatorname{dim}_{g, k}(X, A)$ of the moduli space $\overline{\mathfrak{M}}_{g, k}(X, A ; J)$. 
We denote by

$$
\mathfrak{G}_{g, k}^{0,1}(X, A ; J)=\Gamma\left(\mathfrak{X}_{g, k}(X, A), \Gamma_{g, k}^{0,1}(X, A ; J)\right)
$$

the space of continuous multisections (locally liftable multisections in the sense of $[\mathbf{F u O n}$, Definition 3.5]) $\nu$ such that there exists a collection $\left\{\left(\mathcal{U}_{\alpha}, E_{\alpha}\right)\right\}_{\alpha \in A}$, where

- $\left\{\mathcal{U}_{\alpha}\right\}_{\alpha \in \mathcal{A}}$ is an open cover of $\overline{\mathfrak{M}}_{g, k}(X, A ; J)$ in $\mathfrak{X}_{g, k}(X, A)$ and $E_{\alpha}$ is a finite-rank orbifold subbundle of $\left.\Gamma_{g, k}^{0,1}(X, A ; J)\right|_{\mathcal{U}_{\alpha}}$;

- $\bar{\partial}_{J}^{-1}\left(E_{\alpha}\right)$ is a topological orbifold and $\bar{\partial}_{J}^{-1}\left(E_{\alpha}\right) \cap \mathfrak{X}_{T}(X)$ is a smooth orbifold for every stratum $\mathfrak{X}_{T}(X)$;

- for every $b \in \bar{\partial}_{J}^{-1}\left(E_{\alpha}\right) \cap \bar{\partial}_{J}^{-1}\left(E_{\beta}\right)$, there exists $\gamma \in \mathcal{A}$ such that

$$
x \in \mathcal{U}_{\gamma} \subset \mathcal{U}_{\alpha} \cap \mathcal{U}_{\beta}, \quad E_{\alpha},\left.E_{\beta}\right|_{U_{\gamma}} \subset E_{\gamma},
$$

and the restrictions of $E_{\alpha}$ and $E_{\beta}$ to $\bar{\partial}_{J}^{-1}\left(E_{\gamma}\right) \cap \mathfrak{X}_{T}(X)$ is a smooth orbifold subbundle of the restriction of $E_{\gamma}$;

- the restriction of $E_{\alpha}$ to $\bar{\partial}_{J}^{-1}\left(E_{\alpha}\right) \cap \mathfrak{X}_{T}(X)$ is smooth;

- the restriction of $\nu$ to $\bar{\partial}_{J}^{-1}\left(E_{\alpha}\right) \cap \mathfrak{X}_{T}(X)$ is a smooth multi-section of $E_{\alpha}$.

Collections $\left\{\left(\mathcal{U}_{\alpha}, E_{\alpha}\right)\right\}_{\alpha \in A}$ satisfying the first four conditions are constructed in $[\mathbf{L T}]$ and $[\mathbf{F u O n}]$.

If $\nu$ is a sufficiently small element of $\Gamma_{g, k}^{0,1}(X, A ; J)$, the space

$$
\overline{\mathfrak{M}}_{g, k}(X, A ; J, \nu) \equiv\left\{\bar{\partial}_{J}+\nu\right\}^{-1}(0) \subset \mathfrak{X}_{g, k}(X, A)
$$

is compact, since $\overline{\mathfrak{M}}_{g, k}(X, A ; J)$ is. For a small generic choice of $\nu$, $\overline{\mathfrak{M}}_{g, k}(X, A ; J, \nu)$ admits a stratification by orbifolds of even dimensions; see the first remark below. The main stratum,

$$
\mathfrak{M}_{g, k}^{0}(X, A ; J, \nu)=\overline{\mathfrak{M}}_{g, k}(X, A ; J, \nu) \cap \mathfrak{X}_{g, k}^{0}(X, A),
$$

is a smooth orbifold of dimension $\operatorname{dim}_{g, k}(X, A)$. Since $\mathfrak{X}_{g, k}(X, A)$ is locally a Banach space, there exist arbitrary small neighborhoods $U$ of

$$
\overline{\mathfrak{M}}_{g, k}(X, A ; J, \nu)-\mathfrak{M}_{g, k}^{0}(X, A ; J, \nu)
$$

in $\mathfrak{X}_{g, k}(X, A)$ such that

$$
H_{l}(U ; \mathbb{Q})=\{0\} \quad \forall l \geq \operatorname{dim}_{g, k}(X, A)-1 .
$$

Since $\overline{\mathfrak{M}}_{g, k}(X, A ; J, \nu)-U$ is compact, via the pseudocycle construction of [McSa, Chapter 7] and [RT, Section 1], $\mathfrak{M}_{g, k}^{0}(X, A ; J, \nu)$ determines a homology class

$$
\begin{aligned}
& {\left[\overline{\mathfrak{M}}_{g, k}(X, A ; J, \nu)\right] \in H_{\operatorname{dim}_{g, k}(X, A)}(W, U ; \mathbb{Q})} \\
& \approx H_{\operatorname{dim}_{g, k}(X, A)}(W ; \mathbb{Q}),
\end{aligned}
$$


for any small neighborhood $W$ of $\overline{\mathfrak{M}}_{g, k}(X, A ; J, \nu)$ in $\mathfrak{X}_{g, k}(X, A)$. The isomorphism between the two homology groups is induced by inclusion. Since $\nu$ can be chosen to be arbitrarily small, this procedure defines a rational homology class in an arbitrary small neighborhood of $\overline{\mathfrak{M}}_{g, k}(X, A ; J)$ in $\mathfrak{X}_{g, k}(X, A)$. The Gromov-Witten invariants of $(X, \omega)$ are obtained by cutting down $\overline{\mathfrak{M}}_{g, k}(X, A ; J, \nu)$ by natural cycles on $\mathfrak{X}_{g, k}(X, A)$ to a compact zero-dimensional orbifold contained in the main stratum $\mathfrak{X}_{g, k}^{0}(X, A)$ of $\mathfrak{X}_{g, k}(X, A)$ and taking its cardinality.

Remark 1. The strata of $\overline{\mathfrak{M}}_{1, k}(X, A ; J, \nu)$ locally are unions of finitely many smooth suborbifolds of a smooth orbifold. The branches of the strata correspond to the branches of $\nu$. We will call such objects orbifolds, nevertheless, as these generalized orbifolds are just as suitable for the topological purposes of $[\mathbf{F u O n}],[\mathbf{L T}]$, and this paper; see in $[\mathbf{F u O n}$, Sections 3,4] for details.

Remark 2. The above construction defines a homology class

$$
\Omega_{W} \in H_{\operatorname{dim}_{g, k}(X, A)}(W ; \mathbb{Q})
$$

for every neighborhood $W$ of $\overline{\mathfrak{M}}_{g, k}(X, A ; J)$ in $\mathfrak{X}_{g, k}(X, A)$. Furthermore, if $\iota_{W^{\prime}, W}: W \longrightarrow W^{\prime}$ is the inclusion map of a neighborhood $W$ into a larger neighborhood $W^{\prime}$, then

$$
\iota_{W^{\prime}, W *} \Omega_{W}=\Omega_{W^{\prime}} .
$$

Thus, the above construction defines VFC for $\overline{\mathfrak{M}}_{g, k}(X, A ; J)$ as an element of the inverse limit of the homology groups $H_{*}(W ; \mathbb{Q})$ under inclusion, taken over all neighborhoods of $\overline{\mathfrak{M}}_{g, k}(X, A ; J)$ in $\mathfrak{X}_{g, k}(X, A)$. If $(X, J)$ is algebraic, $\overline{\mathfrak{M}}_{g, k}(X, A ; J)$ is a deformation retract of a neighborhood $W$, and one can then define VFC for $\overline{\mathfrak{M}}_{g, k}(X, A ; J)$ as a homology class in such a neighborhood $W$. However, these formalities are not needed for defining $\mathrm{GW}$-invariants as intersection numbers of $\overline{\mathfrak{M}}_{g, k}(X, A ; J, \nu)$ with certain natural classes on $\mathfrak{X}_{g, k}(X, A)$.

For a small generic perturbation $\nu$ of $\bar{\partial}_{J}$, the closure of $\mathfrak{M}_{g, k}^{0}(X, A ; J, \nu)$ is the entire moduli space $\overline{\mathfrak{M}}_{g, k}(X, A ; J, \nu)$. In particular, the results of $[\mathbf{Z 4}]$, that are summarized in Subsection 1.1, cannot possibly generalize to $\mathfrak{M}_{g, k}^{0}(X, A ; J, \nu)$, even with $g=1$, for a generic $\nu$. Instead, for $g=1$, we consider non-generic perturbations $\nu$ of $\bar{\partial}_{J}$, which we now describe.

An element $[\Sigma ; u]$ of $\mathfrak{X}_{1, k}(X, A)$ is an equivalence class of pairs consisting of a prestable genus-one Riemann surface $\Sigma$ and a $L_{1}^{p}$-map $u: \Sigma \longrightarrow X$. The prestable surface $\Sigma$ is a union of the principal component(s) $\Sigma_{P}$, which is either a smooth torus or a circle of spheres, and trees of rational 
bubble components, which together will be denoted by $\Sigma_{B}$. Let

$$
\mathfrak{X}_{1, k}^{\{0\}}(X, A)=\left\{[\Sigma ; u] \in \mathfrak{X}_{1, k}(X, A): u_{*}\left[\Sigma_{P}\right] \neq 0 \in H_{2}(X ; \mathbb{Z})\right\} .
$$

Suppose

$$
[\Sigma ; u] \in \mathfrak{X}_{1, k}(X, A)-\mathfrak{X}_{1, k}^{\{0\}}(X, A),
$$

i.e. the degree of $\left.u\right|_{\Sigma_{P}}$ is zero. Let $\chi^{0}(\Sigma ; u)$ be the set of components $\Sigma_{i}$ of $\Sigma$ such that for every bubble component $\Sigma_{h}$ that lies between $\Sigma_{i}$ and $\Sigma_{P}$, including $\Sigma_{i}$ itself, the degree of $\left.u\right|_{\Sigma_{h}}$ is zero. The set $\chi^{0}(\Sigma ; u)$ includes the principal component(s) of $\Sigma$. We give an example of the set $\chi^{0}(\Sigma ; u)$ in Figure 2. In this figure, as in Figure 1, we show the domain $\Sigma$ of the stable map $(\Sigma ; u)$ and shade the components of the domain on which the degree of the map $u$ is not zero. Let

$$
\Sigma_{u}^{0}=\bigcup_{i \in \chi^{0}(\Sigma ; u)} \Sigma_{i} .
$$

Definition 1.2. Suppose $(X, \omega)$ is a compact symplectic manifold, $\underline{J} \equiv\left(J_{t}\right)_{t \in[0,1]}$ is a continuous family of $\omega$-tamed almost structures on $X$, $A \in H_{2}(X ; \mathbb{Z})$, and $k \in \overline{\mathbb{Z}}^{+}$. A continuous family of multisections $\underline{\nu} \equiv$ $\left(\nu_{t}\right)_{t \in[0,1]}$, with $\nu_{t} \in \mathfrak{G}_{1, k}^{0,1}\left(X, A ; J_{t}\right)$ for all $t \in[0,1]$, is effectively supported if for every element

$$
b \equiv[\Sigma, u] \in \mathfrak{X}_{1, k}(X, A)-\mathfrak{X}_{1, k}^{\{0\}}(X, A)
$$

there exists a neighborhood $\mathcal{W}_{b}$ of $\Sigma_{u}^{0}$ in a semi-universal family of deformations for $b$ such that

$$
\left.\nu_{t}\left(\Sigma^{\prime} ; u^{\prime}\right)\right|_{\Sigma^{\prime} \cap \mathcal{W}_{b}}=0 \quad \forall\left[\Sigma^{\prime} ; u^{\prime}\right] \in \mathfrak{X}_{1, k}(X, A), t \in[0,1] .
$$

We use the $C^{1}$-topology on the space of all almost complex structures on $X$, in this definition and throughout the rest of the paper. The bundles $\Gamma_{1, k}^{0,1}\left(X, A ; J_{t}\right)$ are contained in the bundle $\Gamma_{1, k}^{1}(X, A)$ over $\mathfrak{X}_{1, k}(X, A)$ with the fibers

$$
\left.\Gamma_{1, k}^{1}(X, A)\right|_{[b]}=\Gamma^{1}(b) / \operatorname{Aut}(b), \text { where } \Gamma^{1}(b)=L^{p}\left(\Sigma ; T^{*} \Sigma \otimes_{\mathbb{R}} u^{*} T X\right),
$$

and with the topology constructed as for $\Gamma_{g, k}^{0,1}(X, A ; J)$. Finally, let $b=[\Sigma ; u]$ be an element of $\mathfrak{X}_{1, k}(X, A)$. A semi-universal universal family of deformations for $b$ is a fibration

$$
\sigma_{b}: \tilde{\mathcal{U}}_{b} \longrightarrow \Delta_{b}
$$

such that $\Delta_{b} / \operatorname{Aut}(b)$ is a neighborhood of $b$ in $\mathfrak{X}_{1, k}(X, A)$ and the fiber of $\sigma_{b}$ over a point $\left[\Sigma^{\prime} ; u^{\prime}\right]$ is $\Sigma^{\prime}$.

If $\underline{\nu}$ is effectively supported and $[\Sigma ; u]$ is as in (1.1), then the restriction of $\nu_{t}$ to a neighborhood $W$ of $\Sigma_{u}^{0}$ in $\Sigma$ is zero for all $t$. Furthermore, 
if $\left\{\left[\Sigma_{k} ; u_{k}\right]\right\}$ is a sequence converging to $[\Sigma ; u]$ in $\mathfrak{X}_{1, k}(X, A)$, then for all $k$ sufficiently large and a choice of representatives $\left(\Sigma_{k} ; u_{k}\right)$ there is an open subset $W_{k}$ of $\Sigma_{k}$ such that $\left.\nu_{t}\left(\Sigma_{k} ; u_{k}\right)\right|_{W_{k}}=0$ and the open sets $W_{k}$ converge to $W$; see the beginning of Section 3 in $[\mathbf{L T}]$ for a detailed setting.

For example, if $[\Sigma ; u]$ is as indicated in Figure 2 and $\underline{\nu}$ is effectively supported, then $\nu_{t}(\Sigma ; u)$ vanishes on a neighborhood of $\Sigma_{P} \cup \Sigma_{h_{3}}$ in $\Sigma$. On the other hand, even if $\Sigma_{h_{2}}$ had not been shaded, i.e. the degree of $\left.u\right|_{\Sigma_{h_{2}}}$ were zero, there still would have been no condition on $\left.\nu_{t}(\Sigma ; u)\right|_{\Sigma_{h_{2}}}$ because the degree of $\left.u\right|_{\Sigma_{h_{1}}}$ is not zero.

If $\underline{J} \equiv\left(J_{t}\right)_{t \in[0,1]}$ is a continuous family of $\omega$-tamed almost structures on $X$, we denote the space of effectively supported families $\underline{\nu}$ as in Definition 1.2 by $\mathfrak{G}_{1, k}^{\text {es }}(X, A ; \underline{J})$. Similarly, if $J$ is an almost complex structure on $X$, we denote by $\mathfrak{G}_{1, k}^{\text {es }}(X, A ; J)$ the subspace of elements $\nu$ of $\mathfrak{G}_{1, k}^{0,1}(X, A ; J)$ such that the family $\nu_{t}=\nu$ is effectively supported.

Remark. Since $\nu$ is a multisection of $\Gamma_{g, k}^{0,1}(X, A ; J)$, which is a union of orbi-vector spaces

$$
\left.\Gamma_{g, k}^{0,1}(X, A ; J)\right|_{[b]}=\Gamma^{0,1}(b ; J) / \operatorname{Aut}(b),
$$

$\nu$ is a family of equivalence classes of elements of $\Gamma_{g, k}^{0,1}(X, A ; J)$ and can be locally represented by a family of elements of $\Gamma^{0,1}(\cdot ; J)$. In order to simplify notation, we will use the same symbol for both, as the exact meaning will be determined by the context.

1.4. Main Results. In this subsection we state the main results of this paper. We begin by describing the subspace $\overline{\mathfrak{M}}_{1, k}^{0}(X, A ; J, \nu)$ of $\overline{\mathfrak{M}}_{1, k}(X, A ; J, \nu)$. We then state the main compactness result, i.e. Theorem 1.4. One of its consequences is that for a small generic choice of $\nu$ the moduli space $\overline{\mathfrak{M}}_{1, k}^{0}(X, A ; J, \nu)$ determines a virtual fundamental class for $\overline{\mathfrak{M}}_{1, k}^{0}(X, A ; J)$, which is independent of $J$; see Theorem 1.5 and Corollary 1.6.

Suppose $[\Sigma ; u]$ is an element of $\mathfrak{X}_{1, k}(X, A)$. Every bubble component $\Sigma_{i} \subset \Sigma_{B}$ is a sphere and has a distinguished singular point, which will be called the attaching node of $\Sigma_{i}$. This is the node of $\Sigma_{i}$ that lies either on $\Sigma_{P}$ or on a bubble $\Sigma_{h}$ that lies between $\Sigma_{i}$ and $\Sigma_{P}$. For example, if $\Sigma$ is as shown in Figure 2, the attaching node of $\Sigma_{h_{3}}$ is the node $\Sigma_{h_{3}}$ shares with the torus. If $[\Sigma ; u]$ is as in $(1.1)$, we denote by $\chi(\Sigma ; u)$ the set of bubble components $\Sigma_{i}$ such that the attaching node of $\Sigma_{i}$ lies on $\Sigma_{u}^{0}$ and the degree of $\left.u\right|_{\Sigma_{i}}$ is not zero, i.e. $\Sigma_{i}$ is not an element of $\chi^{0}(\Sigma ; u)$; see Figure 2. These components are called first-level $(\Sigma ; u)$-effective in 


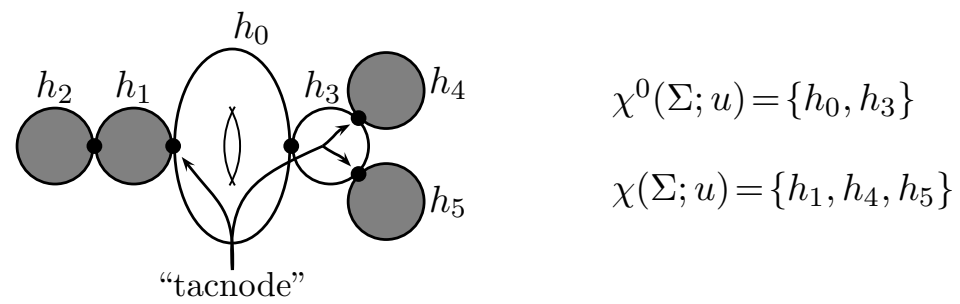

Figure 2. An illustration of Definition 1.3.

[Z4, Subsection 1.2].

Suppose $\nu \in \mathfrak{G}_{1, k}^{\text {es }}(X, A ; J)$ and $[\Sigma ; u]$ is an element of $\overline{\mathfrak{M}}_{1, k}(X, A ; J, \nu)$ as in (1.1). Since $\Sigma_{i} \subset \Sigma_{B}$ is a sphere, we can represent every element of $\mathfrak{X}_{1, k}(X, A)$ by a pair $(\Sigma ; u)$ such that the attaching node of every bubble component $\Sigma_{i} \subset \Sigma_{B}$ is the south pole, or the point $\infty=(0,0,-1)$, of $S^{2} \subset \mathbb{R}^{3}$. Let $e_{\infty}=(1,0,0)$ be a nonzero tangent vector to $S^{2}$ at the south pole. If $i \in \chi(\Sigma ; u)$, we put

$$
\mathcal{D}_{i}(\Sigma ; u)=\left.d\left\{\left.u\right|_{\Sigma_{i}}\right\}\right|_{\infty} e_{\infty} \in T_{\left.u\right|_{\Sigma_{i}}(\infty)} X
$$

Since $\left.u\right|_{\Sigma_{i}}$ is $J$-holomorphic on a neighborhood of $\infty$ in $\Sigma_{i}$, the linear subspace $\mathbb{C} \cdot \mathcal{D}_{i}(\Sigma ; u)$ is determined by $[\Sigma ; u]$, just as in the $\nu=0$ case, which is considered $\left[\mathbf{Z 4}\right.$, Subsection 1.2]. We also note that $\left.u\right|_{\Sigma_{u}^{0}}$ is a degree-zero holomorphic map and thus constant. Thus, $u$ maps the attaching nodes of all elements of $\chi(\Sigma ; u)$ to the same point in $X$, just as in the $\nu=0$ case of $[\mathbf{Z 4}$, Subsection 1.2].

Definition 1.3. Suppose $(X, \omega, J)$ is a compact almost Kähler manifold, $A \in H_{2}(X ; \mathbb{Z})^{*}$, and $k \in \overline{\mathbb{Z}}^{+}$. If $\nu \in \mathfrak{G}_{1, k}^{\text {es }}(X, A ; J)$ is an effectively supported perturbation of the $\bar{\partial}_{J}$-operator, the main component of the space $\overline{\mathfrak{M}}_{1, k}(X, A ; J, \nu)$ is the subset $\overline{\mathfrak{M}}_{1, k}^{0}(X, A ; J, \nu)$ consisting of the elements $[\Sigma ; u]$ of $\overline{\mathfrak{M}}_{1, k}(X, A ; J, \nu)$ such that

(a) the degree of $\left.u\right|_{\Sigma_{P}}$ is not zero, or

(b) the degree of $\left.u\right|_{\Sigma_{P}}$ is zero and

$$
\operatorname{dim}_{\mathbb{C}} \operatorname{Span}_{(\mathbb{C}, J)}\left\{\mathcal{D}_{i}(\Sigma ; u): i \in \chi(\Sigma ; u)\right\}<|\chi(\Sigma ; u)| .
$$

This definition generalizes $[\mathbf{Z 4}$, Definition 1.1]. As in [Z4], we let

$$
H_{2}(X ; \mathbb{Z})^{*}=H_{2}(X ; \mathbb{Z})-\{0\} .
$$

If $[\Sigma ; u]$ is as in $(1.1),[\Sigma ; u]$ belongs to $\overline{\mathfrak{M}}_{1, k}^{0}(X, A ; J, \nu)$ if and only if the branches of $u(\Sigma)$ corresponding to the attaching nodes of the firstlevel effective bubbles of $[\Sigma ; u]$ form a generalized tacnode. In the case of Figure 2, this means that the complex dimension of the span of the images of $d u$ at the attaching nodes of the bubbles $h_{1}, h_{4}$, and $h_{5}$ is at most two. 
Theorem 1.4. Suppose $(X, \omega)$ is a compact symplectic manifold, $\underline{J} \equiv$ $\left(J_{t}\right)_{t \in[0,1]}$ is a continuous family of $\omega$-tamed almost complex structures on $X, A \in H_{2}(X ; \mathbb{Z})^{*}$, and $k \in \overline{\mathbb{Z}}^{+}$. If

$$
\underline{\nu} \equiv\left(\nu_{t}\right)_{t \in[0,1]} \in \mathfrak{G}_{1, k}^{\mathrm{es}}(X, A ; \underline{J})
$$

is a family of sufficiently small perturbations of the $\bar{\partial}_{J_{t}}$-operators on $\mathfrak{X}_{1, k}(X, A)$, then

$$
\overline{\mathfrak{M}}_{1, k}^{0}(X, A ; \underline{J}, \underline{\nu}) \equiv \bigcup_{t \in[0,1]} \overline{\mathfrak{M}}_{1, k}^{0}\left(X, A ; J_{t}, \nu_{t}\right)
$$

is compact.

The requirement that $\nu_{t}$ be sufficiently small means that it lies in a neighborhood of the zero section with respect to a $C^{1}$-type of topology, with appropriate interpretations of the rate of change in the normal directions to the boundary strata of $\mathfrak{X}_{1, k}(X, A)$. This topology will be made apparent in the proof.

Theorem 1.4 follows immediately from Propositions 2.5-2.7; see also the beginning of Subsection 2.3. These propositions generalize $[\mathbf{Z 4}$, Propositions 5.1-5.3].

Theorem 1.5. Suppose $(X, \omega, J)$ is a compact almost Kähler manifold, $A \in H_{2}(X ; \mathbb{Z})^{*}, k \in \overline{\mathbb{Z}}^{+}$, and $W$ is a neighborhood of $\overline{\mathfrak{M}}_{1, k}^{0}(X, A ; J)$ in $\mathfrak{X}_{1, k}(X, A)$. If $\nu \in \mathfrak{G}_{1, k}^{\mathrm{es}}(X, A ; J)$ is a sufficiently small generic perturbation of the $\bar{\partial}_{J}$-operator on $\mathfrak{X}_{1, k}(X, A)$, then $\overline{\mathfrak{M}}_{1, k}^{0}(X, A ; J, \nu)$ determines a rational homology class in $W$. Furthermore, if $\underline{J}=\left(J_{t}\right)_{t \in[0,1]}$ is a family of $\omega$-tamed almost complex structures on $X$, such that $J_{0}=J$ and $J_{t}$ is sufficiently close to $J$ for all $t$, and $\nu_{0}$ and $\nu_{1}$ are sufficiently small generic perturbations of $\bar{\partial}_{J_{0}}$ and $\bar{\partial}_{J_{1}}$ on $\mathfrak{X}_{1, k}(X, A)$, then there exists a homotopy

$$
\underline{\nu}=\left(\nu_{t}\right)_{t \in[0,1]} \in \mathfrak{G}_{1, k}^{\mathrm{es}}(X, A ; \underline{J})
$$

between $\nu_{0}$ and $\nu_{1}$ such that $\overline{\mathfrak{M}}_{1, k}^{0}(X, A ; \underline{J}, \underline{\nu})$ determines a chain in $W$ and

$$
\partial \overline{\mathfrak{M}}_{1, k}^{0}(X, A ; \underline{J}, \underline{\nu})=\overline{\mathfrak{M}}_{1, k}^{0}\left(X, A ; J_{1}, \nu_{1}\right)-\overline{\mathfrak{M}}_{1, k}^{0}\left(X, A ; J_{0}, \nu_{0}\right) .
$$

Corollary 1.6. If $(X, \omega, J)$ is a compact almost Kähler manifold, $A \in H_{2}(X ; \mathbb{Z})^{*}$, and $k \in \overline{\mathbb{Z}}^{+}$, the moduli space $\overline{\mathfrak{M}}_{1, k}^{0}(X, A ; J)$ carries a well-defined virtual fundamental class of the expected dimension. This class is an invariant of $(X, \omega)$.

Proof of Theorem 1.5. It is straightforward to see that for a generic $\nu \in \mathfrak{G}_{1, k}^{\text {es }}(X, A ; J)$ the space $\overline{\mathfrak{M}}_{1, k}(X, A ; J, \nu)$ is stratified by smooth orbifolds of even dimensions. The strata of

$$
\mathfrak{M}_{1, k}^{\{0\}}(X, A ; J, \nu) \equiv \overline{\mathfrak{M}}_{1, k}(X, A ; J, \nu) \cap \mathfrak{X}_{1, k}^{\{0\}}(X, A)
$$


have the expected dimension, based on the index of a certain elliptic operator. In particular, the dimension of the main stratum $\mathfrak{M}_{1, k}^{0}(X, A ; J, \nu)$ is $\operatorname{dim}_{1, k}(X, A)$; the dimensions of all other strata of $\mathfrak{M}_{1, k}^{\{0\}}(X, A ; J, \nu)$ are smaller than $\operatorname{dim}_{1, k}(X, A)$.

On the other hand, suppose $\mathcal{U}_{\mathcal{T}, \nu}(X ; J)$ is a stratum of the complement of $\mathfrak{M}_{1, k}^{\{0\}}(X, A ; J, \nu)$ in $\overline{\mathfrak{M}}_{1, k}(X, A ; J, \nu)$; see Subsection 2.2 for more details. The sets $\chi^{0}(\Sigma ; u)$ and $\chi(\Sigma ; u)$ are independent of the choice of $[\Sigma ; u]$ in $\mathcal{U}_{\mathcal{T}, \nu}(X ; J)$. We denote them by $\chi^{0}(\mathcal{T})$ and $\chi(\mathcal{T})$, respectively. By Definition 1.3, for every $[\Sigma, u] \in \mathcal{U}_{\mathcal{T}, \nu}(X ; J)$ and $i \in \chi^{0}(\mathcal{T}),\left.u\right|_{\Sigma_{i}}$ is constant. Thus,

$$
\mathcal{U}_{\mathcal{T}, \nu}(X ; J) \subset \mathcal{M}_{\mathcal{T}} \times \mathfrak{X}_{\overline{\mathcal{T}}}(X),
$$

where $\mathcal{M}_{\mathcal{T}}$ is a product of $\left|\chi^{0}(\mathcal{T})\right|$ moduli spaces of smooth genus-zero and genus-one curves and $\mathfrak{X}_{\overline{\mathcal{T}}}(X)$ is a certain collection of $|\chi(\mathcal{T})|$-tuples of stable smooth genus-zero bubble maps. For example, if the elements of $\mathcal{U}_{\mathcal{T}, \nu}(X ; J)$ are described by Figure 2 ,

$$
\mathcal{M}_{\mathcal{T}}=\mathcal{M}_{1,2} \times \mathcal{M}_{0,3} .
$$

In this case, $\mathfrak{X}_{\overline{\mathcal{T}}}(X)$ consists of triples of stable genus-zero bubble maps each with a special marked point, corresponding to the attaching nodes of the elements of $\chi(\mathcal{T})$, such that the values of three maps at the special marked points are the same. If $\nu \in \mathfrak{G}_{1, k}^{\text {es }}(X, A ; J)$ is generic, we have a fiber bundle

$$
\pi_{0}: \mathcal{U}_{\mathcal{T}, \nu}(X ; J) \longrightarrow \mathcal{M}_{\mathcal{T}}
$$

with fibers of the expected dimension. An index computation then shows that

$$
\operatorname{dim} \mathcal{U}_{\mathcal{T}, \nu}(X ; J) \leq \operatorname{dim}_{1, k}(X, A)+2(n-|\chi(\mathcal{T})|),
$$

where $2 n$ is the dimension of $X$ as before.

We denote by $E_{\mathcal{T}} \longrightarrow \mathcal{U}_{\mathcal{T}, \nu}(X ; J)$ the direct sum of the $|\chi(\mathcal{T})|$ universal tangent line bundles for the special marked points of the elements of each $\chi(\mathcal{T})$-tuple in $\mathfrak{X}_{\overline{\mathcal{T}}}(X)$ and by

$$
\operatorname{ev}_{P}: \mathcal{U}_{\mathcal{T}, \nu}(X ; J) \longrightarrow X
$$

the map sending an element $[\Sigma ; u]$ of $\mathcal{U}_{\mathcal{T}, \nu}(X ; J)$ to the value of $u$ on $\Sigma_{P}$. Let $\gamma_{E_{\mathcal{T}}} \longrightarrow \mathbb{P} E_{\mathcal{T}}$ be the tautological line bundle. By Definition 1.3,

$$
\mathcal{U}_{\mathcal{T}, \nu}(X ; J) \cap \overline{\mathfrak{M}}_{1, k}^{0}(X, A ; J, \nu)=\pi_{\mathcal{T}}\left(\mathcal{Z}_{\mathcal{T}}\right),
$$

where

$$
\pi_{\mathcal{T}}: \mathbb{P} E_{\mathcal{T}} \longrightarrow \mathcal{U}_{\mathcal{T}, \nu}(X ; J)
$$

is the bundle projection map and $\mathcal{Z}_{\mathcal{T}}$ is the zero set of the section of the vector bundle

$$
\gamma_{E_{\mathcal{T}}}^{*} \otimes \mathrm{ev}_{P}^{*} T X \longrightarrow \mathbb{P} E_{\mathcal{T}}
$$


induced by the differentials $\mathcal{D}_{i}$, with $i \in \chi(\mathcal{T})$, defined above. It is straightforward to see that this section is transverse to the zero set if $\nu \in \mathfrak{G}_{1, k}^{\mathrm{es}}(X, A ; J)$ is generic. Thus,

$$
\begin{aligned}
\operatorname{dim} & \mathcal{U}_{\mathcal{T}, \nu}(X ; J) \cap \overline{\mathfrak{M}}_{1, k}^{0}(X, A ; J, \nu) \leq \operatorname{dim} \mathcal{Z}_{\mathcal{T}} \\
& =\operatorname{dim}_{\mathcal{U}_{\mathcal{T}, \nu}}(X ; J)+2\left(\operatorname{rk}_{\mathbb{C}} E_{\mathcal{T}}-1\right)-2 \operatorname{rk}_{\mathbb{C}}\left(\gamma_{E_{\mathcal{T}}}^{*} \otimes \operatorname{ev}_{P}^{*} T X\right) \\
& \leq \operatorname{dim}_{1, k}(X, A)-2,
\end{aligned}
$$

by (1.2).

By the above, for a generic $\nu \in \mathfrak{G}_{1, k}^{\mathrm{es}}(X, A ; J), \overline{\mathfrak{M}}_{1, k}^{0}(X, A ; J, \nu)$ is stratified by smooth orbifolds of even dimensions, such that the main stratum is of dimension $\operatorname{dim}_{1, k}(X, A)$, while all other strata have smaller dimensions. Thus, the first claim of Theorem 1.5 follows from Theorem 1.4 by the same topological construction as in Subsection 1.3. The second claim of Theorem 1.5 is obtained by a similar argument. q.e.d.

Proof of Corollary 1.6. By the first claim of Theorem 1.5, we can define a homology class for $\overline{\mathfrak{M}}_{1, k}^{0}(X, A ; J)$, which is induced by $\overline{\mathfrak{M}}_{1, k}^{0}(X, A$; $J, \nu)$, for any $J$. By the last statement of Theorem 1.5, this class is independent of the choice $\nu$ and does not change under small changes in $J$. Since the space of $\omega$-tamed almost complex structures on $X$ is pathconnected, it follows that the virtual fundamental class of $\overline{\mathfrak{M}}_{1, k}^{0}(X, A ; J)$ is an invariant of $(X, \omega)$.

q.e.d.

Remark. It is simplest to view the last statement above as the independence of all numbers $\mathrm{GW}_{1, k}^{0 ; X}(\mu)$ obtained by evaluating natural cohomology classes on $\overline{\mathfrak{M}}_{1, k}^{0}(X, A ; J, \nu)$.

\section{Proof of Theorem 1.4}

2.1. Notation: Genus-Zero Maps. We now describe our notation for bubble maps from genus-zero Riemann surfaces and for the spaces of such bubble maps that form the standard stratifications of moduli spaces of stable maps. We also state analogues of Definition 1.2 for genus-zero maps with one and two special marked points.

In general, moduli spaces of stable maps can stratified by the dual graph. However, in the present situation, it is more convenient to make use of linearly ordered sets:

Definition 2.1. (1) A finite nonempty partially ordered set $I$ is a linearly ordered set if for all $i_{1}, i_{2}, h \in I$ such that $i_{1}, i_{2}<h$, either $i_{1} \leq i_{2}$ or $i_{2} \leq i_{1}$. 
(2) A linearly ordered set $I$ is a rooted tree if $I$ has a unique minimal element, i.e. there exists $\hat{0} \in I$ such that $\hat{0} \leq i$ for all $i \in I$.

If $I$ is a linearly ordered set, let $\hat{I}$ be the subset of the non-minimal elements of $I$. For every $h \in \hat{I}$, denote by $\iota_{h} \in I$ the largest element of $I$ which is smaller than $h$, i.e. $\iota_{h}=\max \{i \in I: i<h\}$.

We identify $\mathbb{C}$ with $S^{2}-\{\infty\}$ via the stereographic projection mapping the origin in $\mathbb{C}$ to the north pole, or the point $(0,0,1)$, in $S^{2}$. If $M$ is a finite set, a genus-zero $X$-valued bubble map with $M$-marked points is a tuple

$$
b=(M, I ; x,(j, y), u)
$$

where $I$ is a rooted tree, and

$$
x: \hat{I} \longrightarrow \mathbb{C}=S^{2}-\{\infty\},(j, y): M \longrightarrow I \times \mathbb{C}, u: I \longrightarrow C^{\infty}\left(S^{2} ; X\right)
$$

are maps such that $u_{h}(\infty)=u_{\iota_{h}}\left(x_{h}\right)$ for all $h \in \hat{I}$. We associate such a tuple with Riemann surface

$$
\Sigma_{b}=\left(\bigsqcup_{i \in I} \Sigma_{b, i}\right) / \sim, \quad \Sigma_{b, i}=\{i\} \times S^{2},(h, \infty) \sim\left(\iota_{h}, x_{h}\right) \forall h \in \hat{I},
$$

with marked points

$$
y_{l}(b) \equiv\left(j_{l}, y_{l}\right) \in \Sigma_{b, j_{l}} \quad \text { and } \quad y_{0}(b) \equiv(\hat{0}, \infty) \in \Sigma_{b, \hat{0}}
$$

and continuous map $u_{b}: \Sigma_{b} \longrightarrow X$, given by $\left.u_{b}\right|_{\Sigma_{b, i}}=u_{i}$ for all $i \in I$. The general structure of bubble maps is described by tuples $\mathcal{T}=(M, I ; j, \underline{A})$, where

$$
A_{i}=u_{i *}\left[S^{2}\right] \in H_{2}(X ; \mathbb{Z}) \quad \forall i \in I
$$

We call such tuples bubble types. Let $\mathfrak{X}_{\mathcal{T}}(X)$ denote the subset of $\mathfrak{X}_{0,\{0 \hat{0} \sqcup M}(X, A)$ consisting of stable maps $[\mathcal{C} ; u]$ such that

$$
[\mathcal{C} ; u]=\left[\left(\Sigma_{b},(\hat{0}, \infty),\left(j_{l}, y_{l}\right)_{l \in M}\right) ; u_{b}\right],
$$

for some bubble map $b$ of type $\mathcal{T}$ as above, where $\hat{0}$ is the minimal element of $I$; see [Z2, Section 2] for details. For $l \in\{0\} \sqcup M$, let

$$
\mathrm{ev}_{l}: \mathfrak{X}_{\mathcal{T}}(X) \longrightarrow X
$$

be the evaluation map corresponding to the marked point $y_{l}$.

With notation as above, suppose

$$
[b] \equiv[M, I ; x,(j, y), u] \in \mathfrak{X}_{0,\{0\} \sqcup M}(X, A) .
$$

Let $\chi^{0}(b)$ be the set of components $\Sigma_{b, i}$ of $\Sigma_{b}$ such that for every component $\Sigma_{b, h}$ that lies between $\Sigma_{i}$ and $\Sigma_{b, 0}$, including $\Sigma_{b, i}$ and $\Sigma_{b, 0}$, the degree of $\left.u\right|_{\Sigma_{b, h}}$ is zero. For example, if $b$ is as indicated by Figure 4 on page 431 , the set $\chi^{0}(b)$ consists of the two components that are not 
shaded. The set $\chi^{0}(b)$ is empty if and only if the degree of the restriction of $u_{b}$ to the component containing the special marked point is not zero. Let

$$
\Sigma_{b}^{0}=\{(\hat{0}, \infty)\} \cup \bigcup_{i \in \chi^{0}(b)} \Sigma_{b, i} .
$$

We denote by $\chi(b)$ the set of components $\Sigma_{b, i}$ such that the attaching node of $\Sigma_{b, i}$ lies on $\Sigma_{b}^{0}$ and the degree of $\left.u_{b}\right|_{\Sigma_{b, i}}$ is not zero, i.e. $\Sigma_{b, i}$ is not an element of $\chi^{0}(b)$. If the degree of $\left.u_{b}\right|_{\Sigma_{b, 0}}$ is not zero, $\chi(b)=\{\hat{0}\}$. If $A \neq 0$ and the degree of $\left.u_{b}\right|_{\Sigma_{b, 0}}$ is zero, the set $\chi(b)$ is not empty, but does not contain $\hat{0}$.

Definition 2.2. Suppose $(X, \omega)$ is a compact symplectic manifold, $\underline{J} \equiv\left(J_{t}\right)_{t \in[0,1]}$ is a continuous family of $\omega$-tamed almost structures on $X$, $A \in H_{2}(X ; \mathbb{Z})^{*}$, and $M$ is a finite set. A continuous family of multisections $\underline{\nu} \equiv\left(\nu_{t}\right)_{t \in[0,1]}$, with $\nu_{t} \in \mathfrak{G}_{0,\{0\} \sqcup M}^{0,1}\left(X, A ; J_{t}\right)$ for all $t \in[0,1]$, is effectively supported if for every element $b$ of $\mathfrak{X}_{0,\{0\} \sqcup M}(X, A)$ there exists a neighborhood $\mathcal{W}_{b}$ of $\Sigma_{b}^{0}$ in a semi-universal family of deformations for $b$ such that

$$
\left.\nu_{t}\left(b^{\prime}\right)\right|_{\Sigma_{b^{\prime}} \cap \mathcal{W}_{b}}=0 \quad \forall\left[b^{\prime}\right] \in \mathfrak{X}_{0,\{0\} \sqcup M}(X, A), t \in[0,1] .
$$

Definition 2.3. Suppose $(X, \omega), \underline{J} \equiv\left(J_{t}\right)_{t \in[0,1]}, A$, and $M$ are as in Definition 2.2. A continuous family of multisections $\underline{\nu} \equiv\left(\nu_{t}\right)_{t \in[0,1]}$, with $\nu_{t} \in \mathfrak{G}_{0,\{0,1\} \sqcup M}^{0,1}\left(X, A ; J_{t}\right)$ for all $t \in[0,1]$, is semi-effectively supported if for every element $b$ of $\mathfrak{X}_{0,\{0,1\} \sqcup M}(X, A)$ such that the marked point $y_{1}(b)$ lies on $\Sigma_{b}^{0}$ there exists a neighborhood $\mathcal{W}_{b}$ of $\Sigma_{b}^{0}$ in a semi-universal family of deformations for $b$ such that

$$
\left.\nu_{t}\left(b^{\prime}\right)\right|_{\Sigma_{b^{\prime}} \cap \mathcal{W}_{b}}=0 \quad \forall\left[b^{\prime}\right] \in \mathfrak{X}_{0,\{0,1\} \sqcup M}(X, A), t \in[0,1] .
$$

We denote the spaces of effectively and semi-effectively supported families $\underline{\nu}$ as in Definitions 2.2 and 2.3 by

$$
\mathfrak{G}_{0,\{0\} \sqcup M}^{\text {es }}(X, A ; \underline{J}) \quad \text { and } \quad \mathfrak{G}_{0,\{0,1\} \sqcup M}^{\text {ses }}(X, A ; \underline{J}) \text {, }
$$

respectively. Similarly to the genus-one case, if $J$ is an almost complex structure on $X$, we denote by

$$
\mathfrak{G}_{0,\{0\} \sqcup M}^{\text {es }}(X, A ; J) \quad \text { and } \quad \mathfrak{G}_{0,\{0,1\} \sqcup M}^{\text {ses }}(X, A ; J)
$$

the subspaces of elements $\nu$ of $\mathfrak{G}_{0,\{0\} \sqcup M}^{0,1}(X, A ; J)$ and $\mathfrak{G}_{0,\{0,1\} \sqcup M}^{0,1}(X, A$; $J)$ such that the family $\nu_{t}=\nu$ is effectively supported or semi-effectively supported, respectively. 

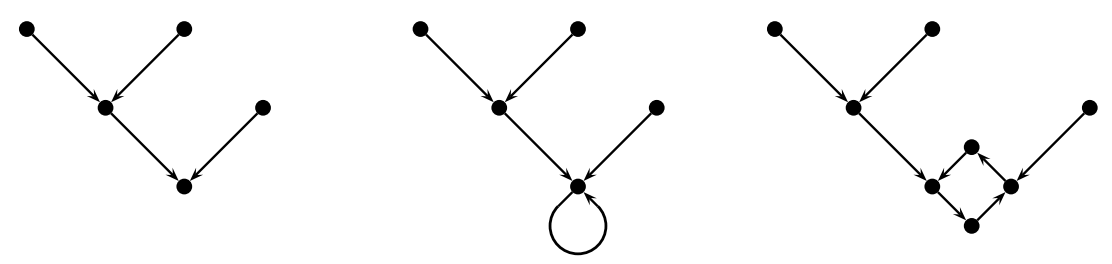

Figure 3. Some enhanced linearly ordered sets.

If $[b]=\left[\Sigma_{b} ; u_{b}\right]$ is an element of $\mathfrak{X}_{0,\{0\} \sqcup M}(X, A)$ is as above and $i \in \chi(b)$, we put

$$
\mathcal{D}_{i} b=\left.d\left\{\left.u_{b}\right|_{\Sigma_{b, i}}\right\}\right|_{\infty} e_{\infty} \in T_{\left.u_{b}\right|_{\Sigma_{b, i}}(\infty)} X .
$$

If $\nu \in \mathfrak{G}_{0,\{0\} \sqcup M}^{\text {es }}(X, A ; J)$ and $b$ is an element of

$$
\overline{\mathfrak{M}}_{0,\{0\} \sqcup M}(X, A ; J, \nu) \equiv\left\{\bar{\partial}_{J}+\nu\right\}^{-1}(0),
$$

then $u_{b} \mid \Sigma_{b, i}$ is $J$-holomorphic on a neighborhood of $\infty$ in $\Sigma_{b, i}$ and $\mathbb{C} \cdot{ }_{J} \mathcal{D}_{i} b$ is determined by $b$, just as in Subsection 1.4. This is also the case if $\nu \in \mathfrak{G}_{0,\{0,1\} \sqcup M}^{\text {ses }}(X, A ; J)$ and $[b]$ is an element of $\overline{\mathfrak{M}}_{0,\{0,1\} \sqcup M}(X, A ; J, \nu)$ such that $y_{1}(b) \in \Sigma_{b}^{0}$. In both of these cases, $\left.u_{b}\right|_{\Sigma_{b}^{0}}$ is a degree-zero holomorphic map and thus constant. Thus, $u_{b}$ maps the attaching nodes of all elements of $\chi(b)$ to the same point in $X$, as in the genus-one case of Subsection 1.4.

2.2. Notation: Genus-One Maps. We next set up analogous notation for maps from genus-one Riemann surfaces. In this case, we also need to specify the structure of the principal component. Thus, we index the strata of $\mathfrak{X}_{1, M}(X, A)$ by enhanced linearly ordered sets:

Definition 2.4. An enhanced linearly ordered set is a pair $(I, \aleph)$, where $I$ is a linearly ordered set, $\aleph$ is a subset of $I_{0} \times I_{0}$, and $I_{0}$ is the subset of minimal elements of $I$, such that if $\left|I_{0}\right|>1$,

$$
\aleph=\left\{\left(i_{1}, i_{2}\right),\left(i_{2}, i_{3}\right), \ldots,\left(i_{n-1}, i_{n}\right),\left(i_{n}, i_{1}\right)\right\}
$$

for some bijection $i:\{1, \ldots, n\} \longrightarrow I_{0}$.

An enhanced linearly ordered set can be represented by an oriented connected graph. In Figure 3, the dots denote the elements of $I$. The arrows outside the loop, if there are any, specify the partial ordering of the linearly ordered set $I$. In fact, every directed edge outside of the loop connects a non-minimal element $h$ of $I$ with $\iota_{h}$. Inside of the loop, there is a directed edge from $i_{1}$ to $i_{2}$ if and only if $\left(i_{1}, i_{2}\right) \in \aleph$.

The subset $\aleph$ of $I_{0} \times I_{0}$ will be used to describe the structure of the principal curve of the domain of stable maps in a stratum of $\mathfrak{X}_{1, M}(X, A)$. 
If $\aleph=\emptyset$, and thus $\left|I_{0}\right|=1$, the corresponding principal curve $\Sigma_{P}$ is a smooth torus, with some complex structure. If $\aleph \neq \emptyset$, the principal components form a circle of spheres:

$$
\Sigma_{P}=\left(\bigsqcup_{i \in I_{0}}\{i\} \times S^{2}\right) / \sim, \quad \text { where }\left(i_{1}, \infty\right) \sim\left(i_{2}, 0\right) \text { if }\left(i_{1}, i_{2}\right) \in \aleph .
$$

A genus-one $X$-valued bubble map with $M$-marked points is a tuple

$$
b=(M, I, \aleph ; S, x,(j, y), u),
$$

where $S$ is a smooth Riemann surface of genus one if $\aleph=\emptyset$ and the circle of spheres $\Sigma_{P}$ otherwise. The objects $x, j, y, u$, and $\left(\Sigma_{b}, u_{b}\right)$ are as in (2.1) and (2.2), except the sphere $\Sigma_{b, \hat{0}}$ is replaced by the genus-one curve $\Sigma_{b ; P} \equiv S$. Furthermore, if $\aleph=\emptyset$, and thus $I_{0}=\{\hat{0}\}$ is a singleelement set, $u_{\hat{0}} \in C^{\infty}(S ; X)$ and $y_{l} \in S$ if $j_{l}=\hat{0}$. In the genus-one case, the general structure of bubble maps is encoded by the tuples of the form $\mathcal{T}=(M, I, \aleph ; j, \underline{A})$. Similarly to the genus-zero case, we denote by $\mathfrak{X}_{\mathcal{T}}(X)$ the subset of $\mathfrak{X}_{1, M}(X, A)$ consisting of stable maps $[\mathcal{C} ; u]$ such that

$$
[\mathcal{C} ; u]=\left[\left(\Sigma_{b},\left(j_{l}, y_{l}\right)_{l \in M}\right) ; u_{b}\right]
$$

for some bubble map $b$ of type $\mathcal{T}$ as above. If $\nu$ is an element of $\mathfrak{G}_{1, M}^{\mathrm{es}}(X, A)$, we put

$$
\mathcal{U}_{\mathcal{T}, \nu}(X ; J)=\left\{[b] \in \mathfrak{X}_{\mathcal{T}}(X):\left\{\bar{\partial}_{J}+\nu\right\}(b)=0\right\} .
$$

All vector orbi-bundles we encounter will be assumed to be normed. Some will come with natural norms; for others, we choose a norm, sometimes implicitly, once and for all. If $\mathfrak{F} \longrightarrow \mathfrak{X}$ is a normed vector bundle and $\delta \in \mathbb{R}^{+}$, let

$$
\mathfrak{F}_{\delta}=\{v \in \mathfrak{F}:|v|<\delta\} .
$$

If $\Omega$ is any subset of $\mathfrak{F}$, we take $\Omega_{\delta}=\Omega \cap \mathfrak{F}_{\delta}$.

2.3. Outline of the Proof of Theorem 1.4. Suppose $(X, \omega)$ is a compact symplectic manifold, $\underline{J} \equiv\left(J_{t}\right)_{t \in[0,1]}$ is a continuous family of $\omega$-tamed almost complex structures on $X, A \in H_{2}(X ; \mathbb{Z})^{*}, M$ is a finite set, and

$$
\underline{\nu} \equiv\left(\nu_{t}\right)_{t \in[0,1]} \in \mathfrak{G}_{1, M}^{\mathrm{es}}(X, A ; \underline{J})
$$

is a family of sufficiently small perturbations of the $\bar{\partial}_{J_{t}}$-operators on $\mathfrak{X}_{1, M}(X, A)$. Let $t_{r}$ and $b_{r}$ be sequences of elements in $[0,1]$ and in $\overline{\mathfrak{M}}_{1, M}^{0}\left(X, A ; J_{t_{r}}, \nu_{t_{r}}\right)$ such that

$$
\lim _{r \longrightarrow \infty} t_{r}=0 \quad \text { and } \quad \lim _{r \longrightarrow \infty} b_{r}=b \in \overline{\mathfrak{M}}_{1, M}\left(X, A ; J_{0}, \nu_{0}\right) .
$$


We need to show that $b \in \overline{\mathfrak{M}}_{1, M}^{0}\left(X, A ; J_{0}, \nu_{0}\right)$. By Definition 1.3 , it is sufficient to assume that $b$ is an element of $\mathcal{U}_{\mathcal{T}, \nu_{0}}\left(X ; J_{0}\right)$ for a bubble type

$$
\mathcal{T}=(M, I, \aleph ; j, \underline{A})
$$

such that $A_{i}=0$ for all minimal elements $i \in I$.

We can also assume that for some bubble type

$$
\mathcal{T}^{\prime}=\left(M, I^{\prime}, \aleph^{\prime} ; j^{\prime}, \underline{A}^{\prime}\right)
$$

$b_{r} \in \mathcal{U}_{\mathcal{T}^{\prime}, \nu_{t_{r}}}\left(X ; J_{t_{r}}\right)$ for all $r$. If $A_{i}^{\prime}=0$ for all minimal elements $i \in I^{\prime}$, the desired conclusion follows Proposition 2.5 below, as it implies that the second condition in Definition 1.3 is closed with respect to the stable map topology. If $A_{i}^{\prime} \neq 0$ for some minimal element $i \in I^{\prime}$ and $\aleph^{\prime} \neq \emptyset$, i.e. the principal component of $\Sigma_{b_{r}}$ is a circle of spheres, Proposition 2.6 implies that $b$ satisfies the second condition in Definition 1.3. Finally, if $\aleph^{\prime}=\emptyset$ and $A_{i}^{\prime} \neq 0$ for the unique minimal element $i$ of $I^{\prime}$, the desired conclusion follows from Proposition 2.7. We note that the three propositions are applied with $b$ and $b_{r}$ that are components of the ones above.

Let $[n]=\{1, \ldots, n\}$.

Proposition 2.5. Suppose $(X, \omega)$ is a compact symplectic manifold, $\underline{J} \equiv\left(J_{t}\right)_{t \in[0,1]}$ is a continuous family of $\omega$-tamed almost complex structures on $X, A \in H_{2}(X ; \mathbb{Z})^{*}, M$ is a finite set, and

$$
\underline{\nu} \equiv\left(\nu_{t}\right)_{t \in[0,1]} \in \mathfrak{G}_{0,\{0\} \sqcup M}^{\mathrm{es}}(X, A ; \underline{J})
$$

is a family of sufficiently small perturbations of the $\bar{\partial}_{J_{t}}$-operators on $\mathfrak{X}_{0,\{0\} \sqcup M}(X, A)$. If $t_{r}$ and $\left[b_{r}\right]$ are sequences of elements in $[0,1]$ and in $\mathfrak{M}_{0,\{0\} \sqcup M}^{0}\left(X, A ; J_{t_{r}}, \nu_{t_{r}}\right)$ such that

$$
\lim _{r \longrightarrow \infty} t_{r}=0 \quad \text { and } \quad \lim _{r \longrightarrow \infty}\left[b_{r}\right]=[b] \in \overline{\mathfrak{M}}_{0,\{0\} \sqcup M}\left(X, A ; J_{0}, \nu_{0}\right),
$$

then either

(a) $\operatorname{dim}_{\mathbb{C}} \operatorname{Span}_{\left(\mathbb{C}, J_{0}\right)}\left\{\mathcal{D}_{i} b: i \in \chi(b)\right\}<|\chi(b)|$, or

(b) $\bigcap_{r=1}^{\infty} \overline{\bigcup_{r^{\prime}>r} \mathbb{C} \cdot J_{r^{\prime}}} \mathcal{D}_{\hat{0}} b_{r^{\prime}} \subset \operatorname{Span}_{\left(\mathbb{C}, J_{0}\right)}\left\{\mathcal{D}_{i} b: i \in \chi(b)\right\}$

Proposition 2.6. Suppose $(X, \omega)$ and $\underline{J}$ are as in Proposition 2.5, $n \in \mathbb{Z}^{+}, A_{1}, \ldots, A_{n} \in H_{2}(X ; \mathbb{Z})^{*}, M_{1}, \ldots, M_{n}$ are finite sets, and for each $k \in[n]$

$$
\underline{\nu}_{k} \equiv\left(\nu_{k, t}\right)_{t \in[0,1]} \in \mathfrak{G}_{0,\{0,1\} \sqcup M_{k}}^{\text {ses }}\left(X, A_{k} ; \underline{J}\right)
$$

is a family of sufficiently small perturbations of the $\bar{\partial}_{J_{t}}$-operators on $\mathfrak{X}_{0,\{0,1\} \sqcup M_{k}}(X, A)$. Let $t_{r}$ and $\left[b_{k, r}\right]$ be sequences of elements in $[0,1]$ 
and in $\mathfrak{M}_{0,\{0,1\} \sqcup M_{k}}^{0}\left(X, A_{k} ; J_{t_{r}}, \nu_{k, t_{r}}\right)$ for $k \in[n]$ such that

$$
\begin{gathered}
\operatorname{ev}_{1}\left(b_{k, r}\right)=\operatorname{ev}_{0}\left(b_{k+1, r}\right) \quad \forall k \in[n-1], \quad \operatorname{ev}_{1}\left(b_{n, r}\right)=\mathrm{ev}_{0}\left(b_{1, r}\right), \\
\lim _{r \longrightarrow \infty} t_{r}=0, \quad \lim _{r \longrightarrow \infty}\left[b_{k, r}\right]=\left[b_{k}\right] \in \overline{\mathfrak{M}}_{0,\{0,1\} \sqcup M_{k}}\left(X, A_{k} ; J_{0}, \nu_{k, 0}\right) \forall k \in[n] .
\end{gathered}
$$

If $y_{1}\left(b_{k}\right) \in \Sigma_{b_{k}}^{0}$ for all $k \in[n]$, then

$$
\operatorname{dim}_{\mathbb{C}} \operatorname{Span}_{\left(\mathbb{C}, J_{0}\right)}\left\{\mathcal{D}_{i} b_{k}: i \in \chi\left(b_{k}\right), k \in[n]\right\}<\sum_{k=1}^{k=n}\left|\chi\left(b_{k}\right)\right| .
$$

Proposition 2.7. Suppose $(X, \omega), \underline{J}, A$, and $M$ are as in Proposition 2.5 and

$$
\underline{\nu} \equiv\left(\nu_{t}\right)_{t \in[0,1]} \in \mathfrak{G}_{1, M}^{\mathrm{es}}(X, A ; \underline{J})
$$

is a family of sufficiently small perturbations of the $\bar{\partial}_{J_{t}}$-operators on $\mathfrak{X}_{1, M}(X, A)$. Let $t_{r}$ and $\left[b_{r}\right]$ be sequences of elements in $[0,1]$ and in $\mathfrak{M}_{1, M}^{0}\left(X, A ; J_{t_{r}}, \nu_{t_{r}}\right)$ such that

$$
\lim _{r \longrightarrow \infty} t_{r}=0 \quad \text { and } \quad \lim _{r \longrightarrow \infty}\left[b_{r}\right]=[b] \in \overline{\mathfrak{M}}_{1, M}\left(X, A ; J_{0}, \nu_{0}\right) .
$$

If $b=(\Sigma ; u)$ is such that the degree of $\left.u\right|_{\Sigma_{P}}$ is zero, then

$$
\operatorname{dim}_{\mathbb{C}} \operatorname{Span}_{\left(\mathbb{C}, J_{0}\right)}\left\{\mathcal{D}_{i} b: i \in \chi(b)\right\}<|\chi(b)| .
$$

Propositions 2.5, 2.6, and 2.7 follow immediately from the estimates (2.12), (2.17), and (2.35) below. These estimates are obtained by combining the approach of $[\mathbf{Z 4}$, Sections 3,4] with some aspects of the local setting of [LT, Section 3]. A key step is [Z4, Lemma 3.5] that gives power series expansions for the behavior of derivatives of $J$-holomorphic genus-zero maps under gluing. They lead to estimates on obstructions to smoothing genus-one $J$-holomorphic maps from singular domains in $[\mathbf{Z 4}$, Lemma 4.4]. While the maps we encounter are not $J$-holomorphic on the entire domain, they are $J$-holomorphic around the part of the domain which is essential for the estimates of [ZZ4, Lemmas 3.5,4.4], i.e. $\Sigma_{b}^{0}$ in the notation of Subsections 1.3 and 2.1 above. The argument in the next two subsections is in fact an extension of [Z4, Section 5], but is far more detailed.

2.4. Proofs of Propositions 2.5 and 2.6. Let $(X, \omega), \underline{J}, A, M, \underline{\nu}$,

$$
b=(M, I ; x,(j, y), u), \quad \text { and }\left.\quad u_{i} \equiv u_{b}\right|_{\Sigma_{b, i}}
$$

be as in the statement of Proposition 2.5. For each $i \in I$, we put

$$
\begin{aligned}
\Gamma(b ; i) & =\left\{\xi \in L_{1}^{p}\left(\Sigma_{b, i} ; u_{i}^{*} T X\right): \xi(\infty)=0\right\}, \\
\Gamma^{0,1}(b ; i) & =L^{p}\left(\Sigma_{b, i} ; \Lambda_{J_{0}, j}^{0,1} T^{*} \Sigma_{b, i} \otimes u_{i}^{*} T X\right),
\end{aligned}
$$

where $j$ is the complex structure on $\Sigma_{b}$. We denote by

$$
D_{J_{0}, \nu_{0} ; b, i}: \Gamma(b ; i) \longrightarrow \Gamma^{0,1}(b ; i)
$$


the linear operator induced by the linearization $D_{J_{0}, \nu_{0} ; b}$ of the section $\bar{\partial}_{J_{0}}+\nu_{0}$ at $b$.

We put

$$
I^{+}=\left\{i \in I: A_{i} \neq 0\right\} .
$$

For each $i \in I^{+}$, choose a finite-dimensional linear subspace

$$
\tilde{\Gamma}_{-}^{0,1}(b ; i) \subset \Gamma\left(\Sigma_{b, i} \times X ; \Lambda_{J_{0}, j}^{0,1} \pi_{1}^{*} T^{*} \Sigma_{b, i} \otimes \pi_{2}^{*} T X\right)
$$

such that

$$
\Gamma^{0,1}(b ; i)=\operatorname{Im} D_{J_{0}, \nu_{0} ; b, i} \oplus\left\{\left\{\operatorname{id} \times u_{i}\right\}^{*} \eta: \eta \in \tilde{\Gamma}_{-}^{0,1}(b ; i)\right\}
$$

and every element of $\tilde{\Gamma}_{-}^{0,1}(b ; i)$ vanishes on a neighborhood of $\infty \in \Sigma_{b, i}$ and the nodes $x_{h}(b) \in \Sigma_{b, i}$ with $\iota_{h}=i$. If $i \in I-I^{+}$, we denote by $\tilde{\Gamma}_{-}^{0,1}(b ; i)$ the zero vector space. Let $\mathcal{T}$ be the bubble type of the map $b$. We put

$$
\begin{aligned}
\tilde{\mathcal{U}}=\left\{b^{\prime} \equiv\left(M, I ; x^{\prime},\left(j, y^{\prime}\right), u^{\prime}\right):\left[b^{\prime}\right]\right. & \in \mathfrak{X}_{\mathcal{T}}(X), \\
\pi_{i}\left\{\bar{\partial}_{J_{0}, j}+\nu_{0}\right\} u_{b^{\prime}} & \left.\in\left\{\operatorname{id} \times u_{i}^{\prime}\right\}^{*} \tilde{\Gamma}_{-}^{0,1}(b ; i) \forall i \in I\right\},
\end{aligned}
$$

where

$$
\pi_{i}: \Gamma^{0,1}\left(b^{\prime} ; J_{0}\right) \longrightarrow \Gamma^{0,1}\left(b^{\prime} ; i\right)
$$

is the natural projection map. By the Implicit Function Theorem, $\tilde{\mathcal{U}}$ is a smooth manifold near $b$. Let

$$
\mathrm{ev}_{0}: \tilde{\mathcal{U}} \longrightarrow X, \quad b^{\prime} \longrightarrow u_{b^{\prime}}(\hat{0}, \infty),
$$

be the evaluation map for the special marked point 0; see also Subsection 2.1. Let

$$
\widetilde{\mathcal{F}} \equiv \tilde{\mathcal{U}} \times \mathbb{C}^{\hat{I}}
$$

be the bundle of smoothing parameters. We denote by $\widetilde{\mathcal{F}}^{\emptyset}$ the subset of $\widetilde{\mathcal{F}}$ consisting of the elements with all components nonzero. For each $v=\left(b^{\prime}, v\right)$, where $b^{\prime} \in \tilde{\mathcal{U}}$ and $v=\left(v_{i}\right)_{i \in \hat{I}}$, and $i \in \chi(b)$, we put

$$
\rho_{i}(v)=\prod_{\hat{0}<h \leq i} v_{h} \in \mathbb{C} \quad \text { and } \quad x_{i}(v)=\sum_{\hat{0}<i^{\prime} \leq i}\left(x_{i^{\prime}}\left(b^{\prime}\right) \prod_{\hat{0}<h<i^{\prime}} v_{h}\right) \in \mathbb{C},
$$

where $x_{i}\left(b^{\prime}\right)$ is the point of $\Sigma_{b^{\prime}, \iota_{i}}$ to which the bubble $\Sigma_{b^{\prime}, i}$ is attached; see (2.2) and Figure 4 on page 431.

For each sufficiently small element $v=\left(b^{\prime}, v\right)$ of $\widetilde{\mathcal{F}}^{\emptyset}$, let

$$
q_{v}: \Sigma_{v} \longrightarrow \Sigma_{b^{\prime}}
$$

be the basic gluing map constructed in [Z2, Subsection 2.2]. In this case, $\Sigma_{v}$ is the projective line $\mathbb{P}^{1}$ with $|M|+1$ marked points. The map $q_{v}$ collapses $|\hat{I}|$ circles on $\Sigma_{v}$. It induces a metric $g_{v}$ on $\Sigma_{v}$ such 
that $\left(\Sigma_{v}, g_{v}\right)$ is obtained from $\Sigma_{b^{\prime}}$ by replacing the $|\hat{I}|$ nodes of $\Sigma_{b^{\prime}}$ by thin necks. Let

$$
u_{v}=u_{b^{\prime}} \circ q_{v} .
$$

We fix a $J_{0}$-compatible metric $g$ on $X$ and denote the corresponding $J_{0}$-compatible connection by $\nabla$. The map $q_{v}$ induces norms $\|\cdot\|_{v, p, 1}$ and $\|\cdot\|_{v, p}$ on the spaces

$$
\Gamma\left(\Sigma_{v} ; u_{v}^{*} T X\right) \quad \text { and } \quad \Gamma\left(\Sigma_{v} ; \Lambda_{J_{0}, j}^{0,1} T^{*} \Sigma_{v} \otimes u_{v}^{*} T X\right),
$$

respectively; see [Z2, Subsection 3.3]. We denote the corresponding completions by $\Gamma(v)$ and $\Gamma^{0,1}(v)$. The norms $\|\cdot\|_{v, p, 1}$ and $\|\cdot\|_{v, p}$ are equivalent to the ones used in [LT, Section 3].

Let $t_{r}$ and $b_{r}$ be as in Proposition 2.5. Since the sequence $\left[b_{r}\right]$ converges to $[b]$, for all $r$ sufficiently large there exist

$$
b_{r}^{\prime} \in \tilde{\mathcal{U}}, \quad v_{r}=\left(b_{r}^{\prime}, v_{r}\right) \in \widetilde{\mathcal{F}}^{\emptyset}, \quad \text { and } \quad \xi_{r} \in \Gamma\left(v_{r}\right)
$$

such that

$$
\begin{gathered}
\lim _{r \longrightarrow \infty} b_{r}^{\prime}=b, \lim _{r \longrightarrow \infty}\left|v_{r}\right|=0, \xi_{r}(\infty)=0 \forall r, \lim _{r \longrightarrow \infty}\left\|\xi_{r}\right\|_{v_{r}, p, 1}=0, \\
\text { and } b_{r} \equiv\left(\Sigma_{b_{r}} ; u_{b_{r}}\right)=\left(\Sigma_{v_{r}} ; \exp _{u_{v_{r}}} \xi_{r}\right) .
\end{gathered}
$$

The last equality holds for a representative $b_{r}$ for $\left[b_{r}\right]$.

Remark. The existence of $b_{r}^{\prime}, v_{r}$, and $\xi_{r}$ as above can be shown by an argument similar to the surjectivity argument in [Z2, Section 4], with significant simplifications. In fact, the only facts about the bubble maps $b_{r}^{\prime}$ we use below are that they are constant on the degree-zero components and holomorphic on fixed neighborhoods of the attaching nodes of the first-level effective bubbles. Such bubble maps $b_{r}^{\prime}$, along with $v_{r}$ and $\xi_{r}$, can be constructed directly from the maps $b_{r}$; see the beginning of Subsection 4.4 in $[\mathbf{Z 2}]$.

If $\delta \in \mathbb{R}^{+}, b^{\prime} \in \tilde{\mathcal{U}}$, and $v=\left(b^{\prime}, v\right) \in \tilde{\mathcal{F}}^{\emptyset}$ is sufficiently small, we put

$$
\Sigma_{b^{\prime}}^{0}(\delta)=\Sigma_{b^{\prime}}^{0} \cup \bigcup_{i \in \chi(b)} A_{b^{\prime}, i}(\delta),
$$

$$
\begin{aligned}
& \text { where } \quad A_{b^{\prime}, i}(\delta)=\left\{(i, z):|z| \geq \delta^{-1 / 2} / 2\right\} \subset \Sigma_{b^{\prime}, i} \approx S^{2} \text {; } \\
& \Sigma_{v}^{0}(\delta)=q_{v}^{-1}\left(\Sigma_{b^{\prime}}^{0}(\delta)\right) \text {. }
\end{aligned}
$$

Choose $\delta \in \mathbb{R}^{+}$such that for all $i \in \chi(b)$ all elements of $\tilde{\Gamma}_{-}^{0,1}(b ; i)$ vanish on $A_{b, i}(2 \delta)$ and for all $r$ sufficiently large

$$
\left.\nu_{t}\left(b_{r}^{\prime}\right)\right|_{\Sigma_{b_{r}^{\prime}}^{0}(2 \delta)}=0 \quad \text { and }\left.\quad \nu_{t}\left(b_{r}\right)\right|_{\Sigma_{v_{r}}^{0}(2 \delta)}=0 \quad \forall t \in[0,1] .
$$


Such a positive number $\delta$ exists by our assumptions on the spaces $\tilde{\Gamma}_{-}^{0,1}(b ; i)$ and the family of perturbations $\underline{\nu}$; see Definition 2.2 .

For every element $b^{\prime}=\left(\Sigma_{b^{\prime}} ; u_{b^{\prime}}\right)$ of $\tilde{\mathcal{U}}$ and every sufficiently small element $v=\left(b^{\prime}, v\right)$ of $\widetilde{\mathcal{F}}^{\emptyset}$, we denote by

$$
\operatorname{Hol}_{J_{0}}\left(\Sigma_{b^{\prime}}^{0}(\delta) ; T_{\mathrm{ev}_{0}\left(b^{\prime}\right)} X\right) \quad \text { and } \quad \operatorname{Hol}_{J_{0}}\left(\Sigma_{v}^{0}(\delta) ; T_{\mathrm{ev}_{0}\left(b^{\prime}\right)} X\right)
$$

the spaces of holomorphic maps from $\Sigma_{b^{\prime}}^{0}(\delta)$ and $\Sigma_{v}^{0}(\delta)$ into the complex vector space $\left(T_{\operatorname{ev}_{0}\left(b^{\prime}\right)} X ; J_{0}\right)$. Let exp be the $\nabla$-exponential map. For every $b^{\prime} \in \tilde{\mathcal{U}}$ as above, $\left.u_{b^{\prime}}\right|_{\Sigma_{b^{\prime}}^{0}}$ is constant and $\left.u_{b^{\prime}}\right|_{\Sigma_{b^{\prime}}^{0}(2 \delta)}$ is $J_{0}$-holomorphic. Thus, if $\delta$ is sufficiently small, there exist continuous families of maps

$$
\Phi_{b^{\prime}} \in L_{1}^{p}\left(\Sigma_{b^{\prime}}^{0}(\delta) ; \operatorname{End}\left(T_{\mathrm{ev}_{0}\left(b^{\prime}\right)} X\right)\right), \quad \vartheta_{b^{\prime}} \in \operatorname{Hol}_{J_{0}}\left(\Sigma_{b}^{0}(\delta) ; T_{\mathrm{ev}_{0}\left(b^{\prime}\right)} X\right)
$$

with $b^{\prime} \in \tilde{\mathcal{U}}$ such that for all $b^{\prime}$ sufficiently close to $b$

$$
\begin{gathered}
\left.\Phi_{b^{\prime}}\right|_{\Sigma_{b^{\prime}}^{0}}=\mathrm{Id}, \quad\left\|\Phi_{b^{\prime}}-\mathrm{Id}\right\|_{b^{\prime}, p, 1} \leq \frac{1}{2}, \quad \text { and } \\
\exp _{\operatorname{ev}_{0}\left(b^{\prime}\right)}\left(\Phi_{b^{\prime}}(z) \vartheta_{b^{\prime}}(z)\right)=u_{b^{\prime}}(z) \quad \forall z \in \Sigma_{b^{\prime}}^{0}(\delta) .
\end{gathered}
$$

This statement follows immediately from the proof of Theorem 2.2 in [FlHS]. Similarly, for every

$$
b_{r} \equiv\left(\Sigma_{b_{r}} ; u_{b_{r}}\right)=\left(\Sigma_{v_{r}} ; \exp _{u_{v_{r}}} \xi_{r}\right)
$$

with $r$ sufficiently large, $\left.u_{b_{r}}\right|_{\Sigma_{v_{r}}^{0}(2 \delta)}$ is $J_{t_{r}}$-holomorphic. Since $\left\|\xi_{r}\right\|_{v_{r}, p, 1}$ tends to zero as $r$ approaches $\infty$, if $\delta$ is sufficiently small and $r$ is sufficiently large, there exist

$$
\Phi_{b_{r}} \in L_{1}^{p}\left(\Sigma_{v_{r}}^{0}(\delta) ; \operatorname{End}\left(T_{\mathrm{ev}_{0}\left(b_{r}^{\prime}\right)} X\right)\right), \quad \vartheta_{b_{r}} \in \operatorname{Hol}_{J_{0}}\left(\Sigma_{v_{r}}^{0}(\delta) ; T_{\mathrm{ev}_{0}\left(b_{r}^{\prime}\right)} X\right)
$$

such that

$$
\begin{gathered}
\left\|\Phi_{b_{r}}-\Phi_{b_{r}^{\prime}} \circ q_{v_{r}}\right\|_{v_{r}, p, 1} \leq C\left(\left\|J_{0}-J_{t_{r}}\right\|_{C^{1}}+\left|v_{r}\right|^{1 / p}+\left\|\xi_{r}\right\|_{v_{r}, p, 1}\right), \\
\exp _{\operatorname{ev}_{0}\left(b_{r}^{\prime}\right)}\left(\Phi_{b_{r}}(z) \vartheta_{b_{r}}(z)\right)=u_{b_{r}}(z) \quad \forall z \in \Sigma_{v_{r}}^{0}(\delta) .
\end{gathered}
$$

In the inequality above, both norms $\|\cdot\|_{v_{r}, p, 1}$ are the norms induced from the pregluing construction as in Subsection 3.3 of $[\mathbf{Z 2}]$. With these norms, the existence of $\Phi_{b_{r}}$ and $\vartheta_{b_{r}}$ follows easily from the proof of Theorem 2.2 in [FlHS]; see the paragraph following Lemma 3.3 in $[\mathbf{Z 4}]$.

If $i \in \chi(b)$ and $b^{\prime} \in \tilde{\mathcal{U}}$, let $w_{i}$ be the standard holomorphic coordinate centered at the point $\infty$ in $\Sigma_{b^{\prime}, i}=S^{2}$. If $m \in \mathbb{Z}^{+}$, we put

$$
\mathcal{D}_{i}^{(m)} \vartheta_{b^{\prime}}=\left.\frac{1}{m !} \frac{d^{m}}{d w_{i}^{m}} \vartheta_{b^{\prime}, i}\left(w_{i}\right)\right|_{w_{i}=0} \in T_{\operatorname{ev}_{0}\left(b^{\prime}\right)} X \text {, where } \vartheta_{b^{\prime}, i}=\left.\vartheta_{b^{\prime}}\right|_{\Sigma_{b^{\prime}, i}} .
$$

Similarly, for all $r$ sufficiently large, we put

$$
\mathcal{D}_{\hat{0}}^{(m)} \vartheta_{b_{r}}=\left.\frac{1}{m !} \frac{d^{m}}{d w^{m}} \vartheta_{b_{r}, i}(w)\right|_{w=0} \in T_{\mathrm{ev}_{0}\left(b_{r}^{\prime}\right)} X,
$$




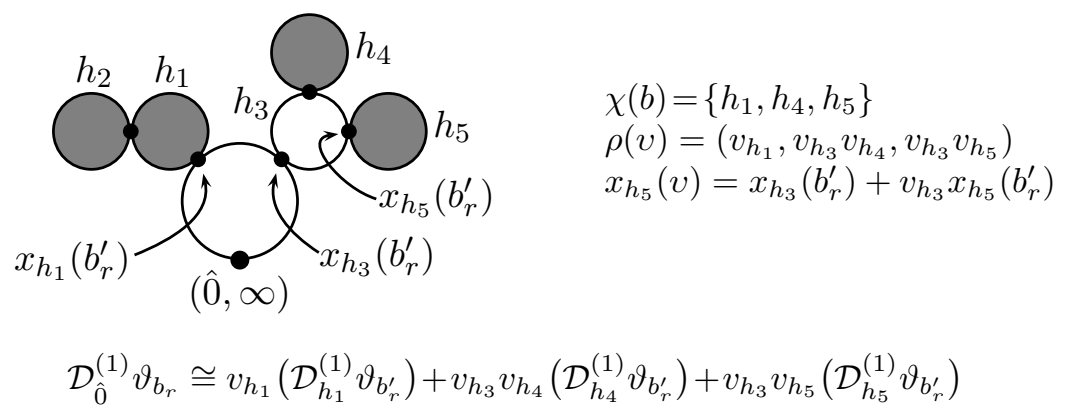

Figure 4. An example of the estimates (2.7) and (2.8).

where $w$ is the standard holomorphic coordinate centered at the point $\infty$ in $\Sigma_{v_{r}} \approx S^{2}$. The key step in the proof of Propositions 2.5 and 2.6 is the power series expansion

$$
\begin{array}{r}
\mathcal{D}_{\hat{0}}^{(m)} \vartheta_{b_{r}}=\sum_{k=1}^{k=m}\left(\begin{array}{c}
m-1 \\
k-1
\end{array}\right) \sum_{i \in \chi(b)} x_{i}^{m-k}\left(v_{r}\right) \rho_{i}^{k}\left(v_{r}\right)\left\{\mathcal{D}_{i}^{(k)} \vartheta_{b_{r}^{\prime}}+\varepsilon_{i, r}^{(k)}\right\} \\
\in\left(T_{\mathrm{ev}_{0}\left(b_{r}^{\prime}\right)} X, J_{0}\right),
\end{array}
$$

for some $\varepsilon_{i, r}^{(k)} \in T_{\operatorname{ev}_{0}\left(b_{r}^{\prime}\right)} X$ such that

$$
\left|\varepsilon_{i, r}^{(k)}\right| \leq C \delta^{-k / 2}\left(\left\|J_{t_{r}}-J_{0}\right\|_{C^{1}}+\left|v_{r}\right|^{1 / p}+\left\|\xi_{r}\right\|_{v_{r}, p, 1}\right) .
$$

The expansion (2.7) is obtained by exactly the same integration-by-parts argument as the expansion in (2a) of Lemma 3.4 in $[\mathbf{Z 4}]$. We point out that $\varepsilon_{i, r}^{(k)}$ is independent of $m$. The $m=1$ case of the estimates (2.7) and (2.8) is illustrated in Figure 4.

We now complete the proof of Proposition 2.5. By the $m=1$ case of (2.7) and (2.8),

$$
\begin{aligned}
\mid \mathcal{D}_{\hat{0}}^{(1)} \vartheta_{b_{r}} & -\sum_{i \in \chi(b)} \rho_{i}\left(v_{r}\right)\left(\mathcal{D}_{i}^{(1)} \vartheta_{b_{r}^{\prime}}\right) \mid \\
\leq & \leq C\left(\left\|J_{t_{r}}-J_{0}\right\|_{C^{1}}+\left|v_{r}\right|^{1 / p}+\left\|\xi_{r}\right\|_{v_{r}, p, 1}\right) \sum_{i \in \chi(b)}\left|\rho_{i}\left(v_{r}\right)\right| .
\end{aligned}
$$

On the other hand, since $\Phi_{b_{r}^{\prime}}(i, \infty)=\operatorname{Id}$ and $\vartheta_{b_{r}^{\prime}}(i, \infty)=0$ for all $i \in \chi(b)$,

$$
\mathcal{D}_{i} b_{r}^{\prime}=\mathcal{D}_{i}^{(1)} \vartheta_{b_{r}^{\prime}} \quad \forall i \in \chi(b) .
$$

Furthermore, since

$$
\begin{aligned}
\left|\Phi_{b_{r}}(\hat{0}, \infty)-\mathrm{Id}\right| & \leq C\left\|\Phi_{b_{r}}-\Phi_{b_{r}^{\prime}} \circ q_{v_{r}}\right\|_{v_{r}, p, 1} \\
& \leq C^{\prime}\left(\left\|J_{0}-J_{t_{r}}\right\|_{C^{1}}+\left|v_{r}\right|^{1 / p}+\left\|\xi_{r}\right\|_{v_{r}, p, 1}\right)
\end{aligned}
$$


and $\vartheta_{b_{r}}(\hat{0}, \infty)=0$,

$$
\left|\mathcal{D}_{\hat{0}} b_{r}-\mathcal{D}_{\hat{0}}^{(1)} \vartheta_{b_{r}}\right| \leq C^{\prime}\left(\left\|J_{0}-J_{t_{r}}\right\|_{C^{1}}+\left|v_{r}\right|^{1 / p}+\left\|\xi_{r}\right\|_{v_{r}, p, 1}\right)\left|\mathcal{D}_{\hat{0}}^{(1)} \vartheta_{b_{r}}\right| .
$$

By (2.9)-(2.11),

$$
\begin{aligned}
& \left|\mathcal{D}_{\hat{0}} b_{r}-\sum_{i \in \chi(b)} \rho_{i}\left(v_{r}\right)\left(\mathcal{D}_{i} b_{r}^{\prime}\right)\right| \\
& \quad \leq C\left(\left\|J_{0}-J_{t_{r}}\right\|_{C^{1}}+\left|v_{r}\right|^{1 / p}+\left\|\xi_{r}\right\|_{v_{r}, p, 1}\right) \sum_{i \in \chi(b)}\left|\rho_{i}\left(v_{r}\right)\right|
\end{aligned}
$$

for all $r$ sufficiently large. Since

$$
\lim _{r \longrightarrow \infty}\left(\left\|J_{0}-J_{t_{r}}\right\|_{C^{1}}+\left|v_{r}\right|^{1 / p}+\left\|\xi_{r}\right\|_{v_{r}, p, 1}\right)=0
$$

and $\mathcal{D}_{i} b_{r}^{\prime} \longrightarrow \mathcal{D}_{i} b$ for all $i \in \chi(b),(2.12)$ implies that $b$ must satisfy one of the two conditions in the statement of Proposition 2.5.

We next complete the proof of Proposition 2.6. By the assumption on the bubble maps $b_{k}$ made in Proposition 2.6 and by Definition 2.3, $\mathrm{ev}_{0}\left(b_{k}\right)=\mathrm{ev}_{1}\left(b_{k}\right)$ for all $k$. Thus,

$$
\mathrm{ev}_{1}\left(b_{k}\right)=\mathrm{ev}_{0}\left(b_{k}\right)=\mathrm{ev}_{1}\left(b_{l}\right) \quad \forall k, l \in[n] .
$$

Let $q$ denote the point $\mathrm{ev}_{0}\left(b_{1}\right)$. We identify a small neighborhood of $q$ in $X$ with a small neighborhood of $q$ in $T_{q} X$ via the exponential map exp and the tangent space to $X$ at a point close to $q$ with $T_{q} X$ via the parallel transport with respect to the $J_{0}$-linear connection $\nabla$.

For each pair $(k, r)$, with $r$ sufficiently large, let $\left(b_{k, r}^{\prime}, v_{k, r}, \xi_{k, r}\right)$ be an analogue of $\left(b_{r}^{\prime}, v_{r}, \xi_{r}\right)$ for $b_{k, r}$. We put

$$
\begin{gathered}
\zeta_{k, r}=\mathrm{ev}_{0}\left(b_{k, r}^{\prime}\right) \in T_{q} X \\
\tilde{\zeta}_{k, r}=\mathrm{ev}_{1}\left(b_{k, r}\right)-\mathrm{ev}_{0}\left(b_{k, r}\right)=\mathrm{ev}_{1}\left(b_{k, r}\right)-\mathrm{ev}_{0}\left(b_{k, r}^{\prime}\right) \in T_{q} X .
\end{gathered}
$$

By the assumption on the maps $b_{k, r}$ in the statement of Proposition 2.6,

$$
\begin{aligned}
& \left|\zeta_{k, r}+\tilde{\zeta}_{k, r}-\zeta_{k+1, r}\right| \leq C\left|\zeta_{k, r}\right| \cdot\left|\tilde{\zeta}_{k, r}\right| \quad \forall k \in[n-1], \\
& \left|\zeta_{n, r}+\tilde{\zeta}_{n, r}-\zeta_{1, r}\right| \leq C\left|\zeta_{n, r}\right| \cdot\left|\tilde{\zeta}_{n, r}\right| ; \\
& \quad \Longrightarrow \quad\left|\tilde{\zeta}_{1, r}+\ldots+\tilde{\zeta}_{n, r}\right| \leq \epsilon_{r} \sum_{k=1}^{k=n}\left|\tilde{\zeta}_{k, r}\right|,
\end{aligned}
$$

for a sequence $\epsilon_{r}$ converging to 0 . 
On the other hand, the marked point $y_{1}\left(b_{k, r}\right)=y_{1}\left(v_{k, r}\right)$ of the bubble map $b_{k, r}$ lies in $\Sigma_{v_{k, r}}^{0}(\delta)$. Thus,

$$
\tilde{\zeta}_{k, r}=\vartheta_{b_{k, r}}\left(y_{1}\left(v_{k, r}\right)\right)=\sum_{m=1}^{\infty} y_{1}\left(v_{k, r}\right)^{-m}\left(\mathcal{D}_{\hat{0}}^{(m)} \vartheta_{b_{k, r}}\right),
$$

where $y_{1}\left(v_{k, r}\right) \in S^{2}-\{\infty\}$ is viewed as a complex number. Combining (2.14) with (2.7) and (2.8) and then taking the lowest-order terms, we obtain an expression of the form

$$
\begin{aligned}
& \left|\tilde{\zeta}_{k, r}-\sum_{i \in \chi\left(b_{k}\right)}\left(y_{1 ; i}\left(b_{k, r}^{\prime}\right)-x_{i ; 1}\left(b_{k, r}^{\prime}\right)\right)^{-1} \rho_{i ; 1}\left(v_{k, r}\right)\left(\mathcal{D}_{i}^{(1)} \vartheta_{b_{k, r}^{\prime}}\right)\right| \\
& \quad \leq C\left(\left\|J_{0}-J_{t_{r}}\right\|_{C^{1}}+\left|v_{k, r}\right|^{1 / p}+\left\|\xi_{k, r}\right\|_{v_{k, r}, p, 1}\right) \sum_{i \in \chi\left(b_{k}\right)}\left|\rho_{i ; 1}\left(v_{k, r}\right)\right| ;
\end{aligned}
$$

see the proof of Corollary 3.7 in $[\mathbf{Z 4}]$ for a derivation and the notation involved. For the present purposes, the only fact we need to know about (2.15) is that

$$
0<\left|\rho_{i ; 1}\left(v_{k, r}\right)\right| \leq\left|v_{k, r}\right| \quad \forall i \in \chi\left(b_{k}\right), k \in[n] .
$$

In particular, $\rho_{i ; 1}\left(v_{k, r}\right)$ is a sequence of nonzero complex numbers that approaches zero as $r$ tends to infinity. By (2.13) and (2.15),

$$
\begin{aligned}
\left|\sum_{k=1}^{k=n} \sum_{i \in \chi\left(b_{k}\right)}\left(y_{1 ; i}\left(b_{k, r}^{\prime}\right)-x_{i ; 1}\left(b_{k, r}^{\prime}\right)\right)^{-1} \rho_{i ; 1}\left(v_{k, r}\right)\left(\mathcal{D}_{i}^{(1)} \vartheta_{b_{k, r}^{\prime}}\right)\right| & \\
& \leq \tilde{\epsilon}_{r} \sum_{k=1}^{k=n} \sum_{i \in \chi\left(b_{k}\right)}\left|\rho_{i ; 1}\left(v_{k, r}\right)\right|
\end{aligned}
$$

for a sequence $\left\{\tilde{\epsilon}_{r}\right\}$ converging to 0 . Since

$$
\mathcal{D}_{i}^{(1)} \vartheta_{b_{k, r}^{\prime}}=\mathcal{D}_{i} b_{k, r}^{\prime} \longrightarrow \mathcal{D}_{i} b_{k} \quad \text { as } \quad r \longrightarrow \infty,
$$

(2.16) and (2.17) imply the conclusion of Proposition 2.6.

2.5. Proof of Proposition 2.7. We prove Proposition 2.7 by combining elements of the previous subsection with a version of the two-stage gluing construction of $[\mathbf{Z 4}$, Section 4]. At the first stage, we smooth out all nodes of an element $\left[\Sigma^{\prime} ; u^{\prime}\right]$ close to $b$ that lie away from the principal component(s) $\Sigma_{P}^{\prime}$ of $\Sigma^{\prime}$. This stage will be unobstructed. The objective of the second stage of the gluing construction is to smooth out the remaining nodes of $\Sigma^{\prime}$. We obtain Proposition 2.7 by estimating the obstruction to achieving this objective.

Let $(X, \omega), \underline{J}, A, M, \underline{\nu}$,

$$
b=(M, I, \aleph ; S, x,(j, y), u), \quad \text { and }\left.\quad u_{i} \equiv u_{b}\right|_{\Sigma_{b, i}}
$$


be as in the statement of Proposition 2.7. For each $i \in I$, we define

$$
D_{J_{0}, \nu_{0} ; b, i}: \Gamma(b ; i) \longrightarrow \Gamma^{0,1}(b ; i)
$$

as at the beginning of the previous subsection. With $I^{+} \subset I$ as in (2.3), choose

$$
\tilde{\Gamma}_{-}^{0,1}(b ; i) \subset \Gamma\left(\Sigma_{b, i} \times X ; \Lambda_{J_{0}, j}^{0,1} \pi_{1}^{*} T^{*} \Sigma_{b, i} \otimes \pi_{2}^{*} T X\right)
$$

as in Subsection 2.4. Let $\mathcal{T}$ be the bubble type of the map $b$. We put

$$
\begin{aligned}
\tilde{\mathcal{U}}=\left\{b^{\prime} \equiv\left(M, I, \aleph ; S^{\prime}, x^{\prime},\left(j, y^{\prime}\right), u^{\prime}\right):\left[b^{\prime}\right] \in \mathfrak{X}_{\mathcal{T}}(X),\right. \\
\left.\pi_{i}\left\{\bar{\partial}_{J_{0}, j}+\nu_{0}\right\} u_{b^{\prime}} \in\left\{\operatorname{id} \times u_{i}^{\prime}\right\}^{*} \tilde{\Gamma}_{-}^{0,1}(b ; i) \forall i \in I\right\},
\end{aligned}
$$

where

$$
\pi_{i}: \Gamma^{0,1}\left(b^{\prime} ; J_{0}\right) \longrightarrow \Gamma^{0,1}\left(b^{\prime} ; i\right)
$$

is the natural projection map. By the Implicit Function Theorem, $\tilde{\mathcal{U}}$ is a smooth manifold near $b$. If $b^{\prime} \in \tilde{\mathcal{U}},\left.u_{b^{\prime}}\right|_{\Sigma_{b^{\prime} ; P}}$ is a degree-zero $J_{0^{-}}$ holomorphic map and thus is constant. Let

$$
\operatorname{ev}_{P}: \tilde{\mathcal{U}} \longrightarrow X, \quad b^{\prime} \longrightarrow u_{b^{\prime}}\left(\Sigma_{b^{\prime} ; P}\right),
$$

be the map sending each element $b^{\prime}$ of $\tilde{\mathcal{U}}$ to the image of the main component of its domain.

For each $b^{\prime} \in \tilde{\mathcal{U}}$, let

$$
\Gamma_{-}\left(b^{\prime}\right)=\left\{\xi \in \Gamma\left(b^{\prime}\right): \pi_{i} D_{J_{0}, \nu_{0} ; b^{\prime}} \xi \in\left\{\operatorname{id} \times u_{b^{\prime}, i}\right\}^{*} \tilde{\Gamma}_{-}^{0,1}(b ; i) \forall i \in I\right\},
$$

where $u_{b^{\prime}, i}=\left.u_{b^{\prime}}\right|_{\Sigma_{b^{\prime}, i}}$. We denote by

$$
\tilde{\Gamma}_{-}^{0,1}\left(b^{\prime} ; X\right) \subset \Gamma\left(\Sigma_{b^{\prime}} \times X ; \Lambda_{J_{0}, j}^{0,1} \pi_{1}^{*} T^{*} \Sigma_{b^{\prime}} \otimes \pi_{2}^{*} T X\right)
$$

the subspace obtained by extending all elements of each of the spaces

$$
\tilde{\Gamma}_{-}^{0,1}\left(b^{\prime} ; i\right) \equiv \tilde{\Gamma}_{-}^{0,1}(b ; i)
$$

with $i \in \hat{I}$ by zero outside of the component $\Sigma_{b^{\prime}, i}$ of $\Sigma_{b^{\prime}}$.

We put

$$
I_{1}=\left\{h \in \hat{I}: \iota_{h} \in I_{0}\right\},
$$

where $I_{0}$ is the subset of minimal elements of $I$. Let

$$
\widetilde{\mathcal{F}} \longrightarrow \tilde{\mathcal{U}}
$$

be the bundle of gluing parameters. In this case, $\widetilde{\mathcal{F}}$ has three distinguished components:

$$
\begin{gathered}
\widetilde{\mathcal{F}}=\widetilde{\mathcal{F}}_{\aleph} \oplus \widetilde{\mathcal{F}}_{0} \oplus \widetilde{\mathcal{F}}_{1}, \quad \text { where } \\
\widetilde{\mathcal{F}}_{\aleph}=\tilde{\mathcal{U}} \times \mathbb{C}^{\aleph}, \quad \widetilde{\mathcal{F}}_{1}=\tilde{\mathcal{U}} \times \mathbb{C}^{\hat{I}-I_{1}},\left.\quad \widetilde{\mathcal{F}}_{0}\right|_{b^{\prime}}=\bigoplus_{h \in I_{1}} T_{x_{h}\left(b^{\prime}\right)} \Sigma_{b^{\prime} ; P} \forall b^{\prime} \in \tilde{\mathcal{U}} .
\end{gathered}
$$

The total space of $\widetilde{\mathcal{F}}_{0}$ has a natural topology; see [Z4, Subsection 2.2]. We denote by $\widetilde{\mathcal{F}}^{\emptyset}$ the subset of $\widetilde{\mathcal{F}}$ consisting of the elements with all 
components nonzero. If $i \in \hat{I}$, let $h(i) \in I_{1}$ be the unique element such that $h(i) \leq i$. For each $v=\left(b^{\prime}, v\right)$, where $b^{\prime} \in \tilde{\mathcal{U}}$ and $v=\left(v_{i}\right)_{i \in \aleph \sqcup \hat{I}}$, and $i \in \chi(b)$, we put

$$
\begin{gathered}
v_{0}=\left(b^{\prime},\left(v_{i}\right)_{i \in \aleph \sqcup I_{1}}\right), \quad v_{1}=\left(b^{\prime},\left(v_{i}\right)_{i \in \hat{I}-I_{1}}\right), \\
\tilde{\rho}_{i}(v)=\prod_{h(i)<h \leq i} v_{h} \in \mathbb{C}, \quad \rho_{i}(v)=\tilde{\rho}_{i}(v) \cdot v_{h(i)} \in T_{x_{h(i)}\left(b^{\prime}\right)} \Sigma_{b^{\prime} ; P} .
\end{gathered}
$$

The component $v_{1}$ of $v$ consists of the smoothings of the nodes of $\Sigma_{b}$ that lie away from the principal component.

For each sufficiently small element $v=\left(b^{\prime}, v\right)$ of $\widetilde{\mathcal{F}}^{\emptyset}$, let

$$
q_{v_{1}}: \Sigma_{v_{1}} \longrightarrow \Sigma_{b^{\prime}}
$$

be the basic gluing map constructed in [Z2, Subsection 2.2]. In this case, the principal component $\Sigma_{v_{1} ; P}$ of $\Sigma_{v_{1}}$ is the same as principal component $\Sigma_{b^{\prime} ; P}$ of $\Sigma_{b^{\prime}}$, and $\Sigma_{v_{1}}$ has $\left|I_{1}\right|$ bubble components $\Sigma_{v_{1}, h}$, with $h \in I_{1}$, attached directly to $\Sigma_{v_{1} ; P}$. The map $q_{v_{1}}$ collapses $\left|\hat{I}-I_{1}\right|$ circles on the bubble components of $\Sigma_{v_{1}}$. It induces a metric $g_{v_{1}}$ on $\Sigma_{v_{1}}$ such that $\left(\Sigma_{v_{1}}, g_{v_{1}}\right)$ is obtained from $\Sigma_{b^{\prime}}$ by replacing $\left|\hat{I}-I_{1}\right|$ nodes by thin necks. Let

$$
u_{v_{1}}=u_{b^{\prime}} \circ q_{v_{1}} \text {. }
$$

The map $q_{v_{1}}$ induces norms $\|\cdot\|_{v_{1}, p, 1}$ and $\|\cdot\|_{v_{1}, p}$ on the spaces

$$
\begin{aligned}
& \left\{\xi \in \Gamma\left(\Sigma_{v_{1}} ; u_{v_{1}}^{*} T X\right):\left.\xi\right|_{\Sigma_{v_{1} ; P}}=0\right\} \quad \text { and } \\
& \left\{\eta \in \Gamma\left(\Sigma_{v_{1}} ; \Lambda_{J_{0}, j}^{0,1} T^{*} \Sigma_{v_{1}} \otimes u_{v_{1}}^{*} T X\right):\left.\eta\right|_{\Sigma_{v_{1} ; P}}=0\right\}
\end{aligned}
$$

respectively; see [Z22, Subsection 3.3]. We denote the corresponding completions by $\Gamma_{B}\left(v_{1}\right)$ and $\Gamma_{B}^{0,1}\left(v_{1}\right)$.

Remark. The weights for the norms $\|\cdot\|_{v_{1}, p, 1}$ and $\|\cdot\|_{v_{1}, p}$ are constructed as [Z2, Subsection 3.3], but on each of the $\left|I_{1}\right|$ bubbles separately. The restrictions of these norms to each of the bubbles are equivalent to the norms used in [LT, Section 3].

Fix $\epsilon_{b} \in \mathbb{R}^{+}$such that for every $h \in I_{1}$ the disk of radius of $8 \epsilon_{b}$ in $\Sigma_{b ; P}$ around the node $x_{h}(b)$ contains no other special, i.e. singular or marked, point of $\Sigma_{b}$. For each $\left(b^{\prime}, v\right) \in \widetilde{\mathcal{F}}^{\emptyset}$ with $b^{\prime} \in \tilde{\mathcal{U}}$ sufficiently close to $b$ and $v$ sufficiently small, let

$$
q_{v_{0} ; 2}: \Sigma_{v} \longrightarrow \Sigma_{v_{1}} \quad \text { and } \quad \tilde{q}_{v_{0} ; 2}: \Sigma_{v} \longrightarrow \Sigma_{v_{1}}
$$

be the basic gluing map of [Z22, Subsection 2.2] corresponding to the gluing parameter $v_{0}$ and the modified basic gluing map defined in the middle of Subsection 4.2 in $[\mathbf{Z 4}]$ with the collapsing radius $\epsilon_{b}$. In this case, $\Sigma_{v}$ is a smooth genus-one curve. For each $h \in I_{1}$, the maps $q_{v_{0} ; 2}$ 
and $\tilde{q}_{v_{0} ; 2}$ collapse the circles of radii $\left|v_{h}\right|^{1 / 2}$ and $\epsilon_{b}$, respectively, around the point $x_{h}\left(b^{\prime}\right) \in \Sigma_{v_{1} ; P}$. Once again, the map

$$
q_{v} \equiv q_{v_{0} ; 2} \circ q_{v_{1}}: \Sigma_{v} \longrightarrow \Sigma_{b^{\prime}}
$$

induces a metric $g_{v}$ on $\Sigma_{v}$ such that $\left(\Sigma_{v}, g_{v}\right)$ is obtained from $\Sigma_{b^{\prime}}$ by replacing all nodes by thin necks.

For each $t \in[0,1]$, let $g_{t}$ be the $J_{t}$-compatible symmetric two-tensor on $X$ given by

$$
g_{t}(\cdot, \cdot)=\frac{1}{2}\left(g\left(J_{0} \cdot, J_{t} \cdot\right)+g\left(J_{t} \cdot, J_{0} \cdot\right)\right)
$$

If $t$ is sufficiently close to $0, g_{t}$ is positive-definite, i.e. it is a $J_{t}$-compatible metric on $X$. We denote the $J_{t}$-compatible connection induced by the Levi-Civita connection of the metric $g_{t}$ by $\nabla^{t}$ and the corresponding exponential map by $\exp ^{t}$.

If $W$ is a small neighborhood of $b$ in $\tilde{\mathcal{U}}$ and $\delta \in \mathbb{R}^{+}$is sufficiently small, let

$$
\begin{aligned}
\mathfrak{X}_{1}(W, \delta)=\left\{\left(\Sigma_{v_{1}} ; u_{v_{1}, \xi}\right) \equiv\left(\Sigma_{v_{1}} ; \exp _{u_{v_{1}}} \xi\right): v\right. & =\left.\left(b^{\prime}, v\right) \in \widetilde{\mathcal{F}}_{\delta}^{\emptyset}\right|_{W} ; \\
\xi & \left.\in \Gamma_{B}\left(v_{1}\right),\|\xi\|_{v_{1}, p, 1} \leq \delta\right\} .
\end{aligned}
$$

For each element $\left(\Sigma_{v_{1}} ; u_{v_{1}, \xi}\right)$ of $\mathfrak{X}_{1}(W, \delta)$, we put

$$
u_{v, \xi}=u_{v_{1}, \xi} \circ \tilde{q}_{v_{0} ; 2} \text {. }
$$

The map $q_{v_{0} ; 2}$ induces norms $\|\cdot\|_{v, p, 1}$ and $\|\cdot\|_{v, p}$ on the spaces

$$
\Gamma\left(\Sigma_{v} ; u_{v, \xi}^{*} T X\right) \quad \text { and } \quad \Gamma\left(\Sigma_{v} ; \Lambda_{J_{t}, j}^{0,1} T^{*} \Sigma_{v} \otimes u_{v, \xi}^{*} T X\right),
$$

respectively. For $t$ sufficiently small, we use the metric $g_{t}$ on $X$ to define a norm on the latter space. Let $\Gamma(v ; \xi)$ and $\Gamma_{t}^{0,1}(v ; \xi)$ be the corresponding completions. If $v=\left(b^{\prime}, v\right)$, we put

$$
\Gamma_{-}(v ; \xi)=\left\{\left(\Pi_{\xi}\left(\zeta \circ q_{v_{1}}\right)\right) \circ \tilde{q}_{v_{0} ; 2}: \zeta \in \Gamma_{-}\left(b^{\prime}\right)\right\} \subset \Gamma(v ; \xi),
$$

where $\Pi_{\xi}$ is the $\nabla$-parallel transport along the $\nabla$-geodesics $\tau \rightarrow \exp _{u_{v_{1}}} \tau \xi$. Let

$$
\Gamma_{+}(v ; \xi) \subset \Gamma(v ; \xi)
$$

be the $L^{2}$-orthogonal complement of $\Gamma_{-}(v ; \xi)$ defined with respect to the metrics $g_{v}$ on $\Sigma_{v}$ and $g$ on $X$. For every $t \in[0,1]$, we denote by

$$
\pi_{t}^{0,1}: \Gamma\left(\Sigma_{v} ; T^{*} \Sigma_{v} \otimes_{\mathbb{R}} u_{v, \xi}^{*} T X\right) \longrightarrow \Gamma\left(\Sigma_{v} ; \Lambda_{J_{t}, j}^{0,1} T^{*} \Sigma_{v} \otimes u_{v, \xi}^{*} T X\right)
$$

the natural projection map.

For each $b^{\prime} \in \tilde{\mathcal{U}}$, each sufficiently small element $v=\left(b^{\prime}, v\right)$ of $\widetilde{\mathcal{F}}^{\emptyset}$, and $\delta \in \mathbb{R}^{+}$, we define

$$
A_{b^{\prime}, i}(\delta), \Sigma_{b^{\prime}}^{0}(\delta) \subset \Sigma_{b^{\prime}}, \quad \Sigma_{v_{1}}^{0}(\delta) \subset \Sigma_{v_{1}}, \quad \text { and } \quad \Sigma_{v}^{0}(\delta) \subset \Sigma_{v}
$$


by (2.5) and (2.6). Choose a neighborhood $W$ of $b$ in $\tilde{\mathcal{U}}, \delta_{b} \in\left(0, \epsilon_{b}\right)$, and $\delta \in\left(0, \delta_{b}^{2} / 2\right)$ such that

(i) the maps $q_{v}$ and $\tilde{q}_{v_{0} ; 2}$ are defined for all $\left.v \in \widetilde{\mathcal{F}}_{\delta}^{\emptyset}\right|_{W}$;

(ii) for all $i \in \chi(b)$ all elements of $\tilde{\Gamma}_{-}^{0,1}(b ; i)$ vanish on $A_{b, i}\left(\delta_{b}\right)$;

(iii) $\left.\nu_{t}\left(b^{\prime}\right)\right|_{\Sigma_{b^{\prime}}^{0}\left(2 \delta_{b}\right)}=0$ for every $t \in[0,1]$ and $b^{\prime} \in W$;

(iv) $\left.\nu_{t}(\tilde{b})\right|_{\Sigma_{v}^{0}\left(2 \delta_{b}\right)}=0$ for every $t \in[0,1]$ and every $\tilde{b}=\left(\Sigma_{v} ; \exp _{u_{v_{1}, \xi}} \zeta\right)$ such that

$\left.v \in \widetilde{\mathcal{F}}_{\delta}^{\emptyset}\right|_{W},\left(\Sigma_{v_{1}} ; u_{v_{1}, \xi}\right) \in \mathfrak{X}_{1}(W, \delta), \zeta \in \Gamma(v ; \xi)$, and $\|\zeta\|_{v, p, 1} \leq \delta$.

Such a positive number $\delta$ exists by our assumptions on the spaces $\tilde{\Gamma}_{-}^{0,1}(b ; i)$ and the family of perturbations $\underline{\nu}$; see Definition 1.2 .

Suppose $\left.v \in \widetilde{\mathcal{F}}_{\delta}^{\emptyset}\right|_{W}$ and $\left(\Sigma_{v_{1}} ; u_{v_{1}, \xi}\right) \in \mathfrak{X}_{1}(W, \delta)$. By the construction of the map $\tilde{q}_{v_{0} ; 2}$ in Subsection 4.2 of $[\mathbf{Z} 4]$ and the assumptions that $\delta \leq \delta_{b}^{2} / 2$ and $\delta_{b} \leq \epsilon_{b}$,

$$
\tilde{q}_{v_{0} ; 2}: \Sigma_{v}-\Sigma_{v}^{0}\left(\delta_{b} / 2\right) \longrightarrow \Sigma_{v_{1}}-\Sigma_{v_{1}}^{0}\left(\delta_{b} / 2\right)
$$

is a biholomorphism. Thus, by the assumption (iv) above with $\zeta=0$, for every $t \in[0,1]$, we can define

$$
\begin{gathered}
\nu_{t ; v_{1}, \xi} \in \Gamma^{0,1}\left(\Sigma_{v_{1}} ; \Lambda_{J, j}^{0,1} T^{*} \Sigma_{v_{1}} \otimes u_{v_{1}, \xi}^{*} T X\right) \quad \text { by } \\
\tilde{q}_{v_{0} ; 2}^{*} \nu_{t ; v_{1}, \xi}=\nu_{t}\left(\Sigma_{v} ; u_{v, \xi}\right),\left.\nu_{t ; v_{1}, \xi}\right|_{\Sigma_{v_{1}}^{0}\left(\delta_{b}\right)}=0 .
\end{gathered}
$$

If $v=\left(b^{\prime}, v\right)$, we put

$$
\begin{aligned}
\Gamma_{t, \delta}(v)=\left\{\xi \in \Gamma_{B}\left(v_{1}\right):\|\xi\|_{v_{1}, p, 1} \leq \delta\right. & \\
& \left.\left\{\bar{\partial}_{J}+\nu_{t ; v_{1}, \xi}\right\} u_{v_{1}, \xi} \in \pi_{t}\left\{q_{v_{1}} \times u_{v_{1}, \xi}\right\}^{*} \tilde{\Gamma}_{-}^{0,1}\left(b^{\prime} ; X\right)\right\} .
\end{aligned}
$$

Let $t_{r}$ and $b_{r}$ be as in Proposition 2.7. Since the sequence $\left[b_{r}\right]$ converges to $[b]$, for all $r$ sufficiently large there exist

$$
b_{r}^{\prime} \in W, \quad v_{r}=\left(b_{r}^{\prime}, v_{r}\right) \in \tilde{\mathcal{F}}_{\delta}^{\emptyset}, \quad \xi_{r} \in \Gamma_{t, \delta}\left(v_{r}\right), \quad \zeta_{r} \in \Gamma_{+}\left(v_{r} ; \xi_{r}\right)
$$

such that

$$
\begin{gathered}
\lim _{r \longrightarrow \infty} b_{r}^{\prime}=b, \quad \lim _{r \longrightarrow \infty}\left|v_{r}\right|=0, \quad \lim _{r \longrightarrow \infty}\left\|\xi_{r}\right\|_{v_{r ; 1}, p, 1}=0, \\
\lim _{r \longrightarrow \infty}\left\|\zeta_{r}\right\|_{v_{r}, p, 1}=0, \quad b_{r} \equiv\left(\Sigma_{b_{r}} ; u_{b_{r}}\right)=\left(\Sigma_{v_{r}} ; \exp _{u_{v_{r}, \xi_{r}}}^{t} \zeta_{r}\right) .
\end{gathered}
$$

The last equality holds for a representative $b_{r}$ for $\left[b_{r}\right]$. The existence of $\left(v_{r}, \xi_{r}, \zeta_{r}\right)$ for

$$
b_{r} \in \mathfrak{M}_{1, M}^{0}\left(X, A ; J_{t_{r}}, \nu_{t_{r}}\right)
$$

as above will imply that $b$ satisfies the second property in Definition 1.3.

Remark. Similarly to the genus-zero case, the existence of $\left(v_{r}, \xi_{r}, \zeta_{r}\right)$ can be shown by a variation on the surjectivity argument of $[\mathbf{Z 2}$, Section 4]; see also the paragraph following Lemma 4.4 in [Z4]. This is also 
the case if the map $q_{v_{0} ; 2}$ is used instead of $\tilde{q}_{v_{0} ; 2}$ in (2.18) . However, using the map $\tilde{q}_{v_{0} ; 2}$ in (2.18) makes the maps $u_{v, \xi}$, with $\xi \in \Gamma_{t, \delta}(v)$, closer to being $\left(J_{t}, \nu_{t}\right)$-holomorphic. Since $\left.u_{b^{\prime}}\right|_{\Sigma_{b^{\prime} ; P}}$ is constant, the choice of $\tilde{q}_{v_{0} ; 2}$ for constructing approximately $\left(J_{t}, \nu_{t}\right)$-holomorphic maps is analogous to that of Section 3 in $[\mathbf{L T}]$.

For each $\left.v \in \tilde{\mathcal{F}}_{\delta}^{\emptyset}\right|_{W}$ and $\xi \in \Gamma_{t, \delta}(v)$, let

$$
D_{v ; \xi}^{t}: \Gamma(v ; \xi) \longrightarrow \Gamma_{t}^{0,1}(v ; \xi)
$$

be the linearization of section $\bar{\partial}_{J_{t}}+\nu_{t}$ at $\left(\Sigma_{v} ; u_{v, \xi}\right)$ defined via the connection $\nabla^{t}$. We denote by

$$
\Gamma_{t ;+}^{0,1}(v ; \xi) \subset \Gamma_{t}^{0,1}(v ; \xi)
$$

the image $\Gamma_{+}(v ; \xi)$ under $D_{v ; \xi}^{t}$. By the same argument as in $[\mathbf{Z 2}$, Subsection 5.4], there exists $C \in \mathbb{R}^{+}$such that

$$
\begin{gathered}
C^{-1}\|\zeta\|_{v, p, 1} \leq\left\|D_{v ; \xi}^{t} \zeta\right\|_{v, p} \leq C\|\zeta\|_{v, p, 1} \\
\forall t \in[0, \delta],\left.v \in \widetilde{\mathcal{F}}_{\delta}^{\emptyset}\right|_{W}, \quad \xi \in \Gamma_{t, \delta}(v), \quad \zeta \in \Gamma_{+}(v ; \xi),
\end{gathered}
$$

provided $W$ and $\delta$ are sufficiently small.

Put

$$
\tilde{\Gamma}_{t ;-}^{0,1}(v ; \xi)=\left\{\pi_{t}\left\{q_{v} \times u_{v, \xi}\right\}^{*} \eta: \eta \in \tilde{\Gamma}_{-}^{0,1}\left(b^{\prime} ; X\right)\right\} \subset \Gamma_{t}^{0,1}(v ; \xi) .
$$

If $\delta$ is sufficiently small, by the same argument as in [Z2, Subsection 3.5] and our assumptions on the spaces $\tilde{\Gamma}_{-}(b ; i)$,

$$
\Gamma_{t}^{0,1}(v ; \xi)=\Gamma_{t ;+}^{0,1}(v ; \xi) \oplus \tilde{\Gamma}_{t ;-}^{0,1}(v ; \xi) \oplus \Gamma_{t ;-}^{0,1}(v ; \xi),
$$

for some subspace $\Gamma_{t ;-}^{0,1}(v ; \xi)$ of $\Gamma_{t}^{0,1}(v ; \xi)$ isomorphic to the cokernel of the composition:

$$
\pi \circ D_{J_{0}, \nu_{0} ; b^{\prime}}: \Gamma\left(b^{\prime}\right) \longrightarrow \Gamma^{0,1}\left(b^{\prime} ; J_{0}\right) \longrightarrow \Gamma^{0,1}\left(b^{\prime} ; J_{0}\right) /\left\{\mathrm{id} \times u_{b^{\prime}}\right\}^{*} \tilde{\Gamma}_{-}^{0,1}\left(b^{\prime} ; X\right) .
$$

This cokernel is naturally isomorphic to

$$
\Gamma_{-}^{0,1}\left(b^{\prime} ; J_{0}\right) \equiv \mathcal{H}_{b^{\prime}}^{0,1} \otimes_{J_{0}} T_{\mathrm{ev}_{P}\left(b^{\prime}\right)} X \subset \Gamma^{0,1}\left(b^{\prime} ; J_{0}\right),
$$

where $\mathcal{H}_{b^{\prime}}^{0,1}$ is the one-dimensional complex vector space of $(0,1)$-harmonic forms on the principal component $\Sigma_{b^{\prime} ; P}$ of $\Sigma_{b^{\prime}}$. If $\Sigma_{b^{\prime} ; P}$ is a circle of spheres, the elements of $\mathcal{H}_{b^{\prime}}^{0,1}$ have simple poles at the nodes of $\Sigma_{b^{\prime} ; P}$ with the residues adding up to zero at each node. Recall that $\left(\Sigma_{v}, g_{v}\right)$ is obtained from $\Sigma_{b^{\prime}}$ by replacing the nodes of $\Sigma_{b^{\prime}}$ with thin necks. The map

$$
q_{v}: \Sigma_{v} \longrightarrow \Sigma_{b^{\prime}}
$$

collapses each neck at its thinnest position to the corresponding node. For each element $\eta$ of $\Gamma_{-}^{0,1}\left(b^{\prime} ; J_{t}\right)$, we can construct an element $R_{v, \xi} \eta$ of $\Gamma_{t}^{0,1}(v ; \xi)$ by parallel transporting $\eta$ along the restriction of $u_{v, \xi}$ to 
$q_{v}^{-1}\left(A_{b, h}\left(\delta_{b}^{2}\right)\right)$ for each $h \in I_{1}$ and cutting it off with a smooth function that drops from 1 to 0 over the annulus $q_{v}^{-1}\left(A_{b, h}\left(\delta_{b}^{2}\right)-A_{b, h}\left(\delta_{b}^{2} / 4\right)\right)$; see the middle of Subsection 4.2 in $[\mathbf{Z 4}]$ for details. We denote by $\Gamma_{t ;-}^{0,1}(v ; \xi)$ the image of $\Gamma_{-}^{0,1}\left(b^{\prime} ; J_{t}\right)$ under $R_{v}$.

Remark. In the construction of the map $\tilde{q}_{v_{0} ; 2}$ in [Z4, Subsection 4.2], $\delta_{K}$ corresponds to $\epsilon_{b}^{2}$ above. In the construction of $R_{v, \xi} \eta$ in $[\mathbf{Z 4}$, Subsection 4.2$], \delta_{K}$ corresponds to $\delta_{b}^{2} / 4$ above.

Let $\left\langle\langle\cdot, \cdot\rangle_{t}\right.$ denote the Hermitian inner-product on $\Gamma_{t}^{0,1}(v ; \xi)$ induced by the $J_{t}$-compatible metric $g_{t}$ on $X$. For each $\eta \in \Gamma_{-}^{0,1}\left(b^{\prime} ; J_{t}\right)$, let

$$
\|\eta\|=\sum_{h \in I_{1}}\left\langle\eta_{x_{h}\left(b^{\prime}\right)}, \eta_{x_{h}\left(b^{\prime}\right)}\right\rangle_{t}
$$

where $\langle\cdot, \cdot\rangle_{t}$ is the hermitian inner-product on $\mathcal{H}_{b^{\prime}}^{0,1} \otimes_{J_{t}} T_{\mathrm{ev}_{P}\left(b^{\prime}\right)} X$ defined via the metric $g$ on $X$ and the original metric $g_{b^{\prime}}$ on $\Sigma_{b^{\prime}}$. From the construction of $R_{v}$ in [Z4, Subsection 4.2] and Holder's inequality, it is immediate that

$$
\left|\left\langle\left\langle\eta^{\prime}, R_{v, \xi} \eta\right\rangle\right\rangle_{t}\right| \leq C\|\eta\|\left\|\eta^{\prime}\right\|_{v, p} \quad \forall \eta \in \Gamma_{-}^{0,1}\left(b^{\prime} ; J_{t}\right), \eta^{\prime} \in \Gamma_{t}^{0,1}(v ; \xi) ;
$$

see $[\mathbf{Z 4},(4.11)]$. Another essential property of the above construction is that

$$
\begin{gathered}
\left|\left\langle\left\langle D_{v ; \xi}^{t} \zeta, R_{v, \xi} \eta\right\rangle\right\rangle_{t}\right| \leq C|v|^{1 / 2}\|\eta\|\|\zeta\|_{v, p, 1} \\
\forall t \in[0, \delta],\left.v \in \widetilde{\mathcal{F}}_{\delta}^{\emptyset}\right|_{W}, \quad \xi \in \Gamma_{t, \delta}(v), \quad \zeta \in \Gamma(v ; \xi) ;
\end{gathered}
$$

see part (7) of Lemma 4.4 in $[\mathbf{Z 4}]$.

Due to the assumption (2.20), the condition (2.21) is equivalent to

$$
\left\{\bar{\partial}_{J_{t_{r}}}+\nu_{t_{r}}\right\} u_{v_{r}, \xi_{r}}+D_{v_{r} ; \xi_{r}}^{t_{r}} \zeta_{r}+N_{v_{r} ; \xi_{r}}^{t_{r}} \zeta_{r}=0
$$

The quadratic term $N_{v ; \xi}^{t}$ satisfies

$$
\left\|N_{v ; \zeta}^{t} \zeta\right\|_{v, p} \leq C\|\zeta\|_{v, p, 1}^{2}
$$

$$
\forall t \in[0, \delta],\left.v \in \widetilde{\mathcal{F}}_{\delta}^{\emptyset}\right|_{W}, \quad \xi \in \Gamma_{t, \delta}(v), \quad \zeta \in \Gamma(v ; \xi) \text { s.t. }\|\zeta\|_{v, p, 1} \leq \delta .
$$

We will obtain Proposition 2.7 by estimating the inner-product $\langle\langle\cdot, \cdot\rangle\rangle_{t_{r}}$ of each term in $(2.26)$ with each element of $\Gamma_{t_{r} ;-}^{0,1}\left(v_{r} ; \xi_{r}\right)$.

First, for every $\left.v \in \widetilde{\mathcal{F}}_{\delta}^{\emptyset}\right|_{W}$ and $\xi \in \Gamma_{t, \delta}(v)$, let

$$
\tilde{\pi}_{v ; \xi}^{t ;+}: \Gamma_{t}^{0,1}(v ; \xi) \longrightarrow \Gamma_{t ;+}^{0,1}(v ; \xi) \oplus \Gamma_{t ;-}^{0,1}(v ; \xi)
$$


be the projection map corresponding to the decomposition (2.23). Then,

$$
\begin{aligned}
\tilde{\pi}_{v ; \xi}^{t ;+}\{ & \left.\bar{\partial}_{J_{t}}+\nu_{t}\right\} u_{v, \xi}=\tilde{\pi}_{v ; \xi}^{t ;+}\left\{\bar{\partial}_{J_{t}}+\tilde{q}_{v_{0} ; 2}^{*} \nu_{t ; v_{1}, \xi}\right\}\left(u_{v_{1}, \xi} \circ \tilde{q}_{v_{0} ; 2}\right) \\
& =\tilde{\pi}_{v ; \xi}^{t ;+}\left(\left(\left\{\bar{\partial}_{J_{t}}+\nu_{t ; v_{1}, \xi}\right\} u_{v_{1}, \xi}\right) \circ \partial \tilde{q}_{v_{0} ; 2}\right)+\tilde{\pi}_{v ; \xi}^{t ;+} \bar{\partial}_{J_{t}}\left(u_{v, \xi} \circ \tilde{q}_{v_{0} ; 2}\right) \\
& =\tilde{\pi}_{v ; \xi}^{t ;+} \bar{\partial}_{J_{t}}\left(u_{v, \xi} \circ \tilde{q}_{v_{0} ; 2}\right) .
\end{aligned}
$$

The reason for the second equality is that the map $\tilde{q}_{v_{0} ; 2}$ is holomorphic over the support of $\nu_{t ; v_{1}, \xi}$. This last equality follows from the definition of $\Gamma_{t, \delta}(v)$ above. By our assumptions on $b^{\prime}$ and $\xi$,

$$
\left.u_{v_{1}, \xi}\right|_{q_{v_{1}}^{-1}\left(\Sigma_{b^{\prime} ; P}\right)}=\text { const }\left.\quad \Longrightarrow \quad d u_{v_{1}, \xi} \circ \bar{\partial} \tilde{q}_{v_{0} ; 2}\right|_{\tilde{q}_{v_{0} ; 2}^{-1} q_{v_{1}}^{-1}\left(\Sigma_{b^{\prime} ; P}\right)}=0 .
$$

By the construction of the map $\tilde{q}_{v_{0} ; 2}$, the restriction of $\tilde{q}_{v_{0} ; 2}$ to the complement of $\tilde{q}_{v_{0} ; 2}^{-1} q_{v_{1}}^{-1}\left(\Sigma_{b^{\prime} ; P}\right)$ in $\Sigma_{v}$ is holomorphic outside of the annuli

$$
\mathcal{A}_{v, h} \equiv \tilde{q}_{v_{0} ; 2}^{-1} q_{v_{1}}^{-1}\left(A_{b^{\prime}, h}\left(2\left|v_{h}\right|^{2} / \epsilon_{b}^{2}\right)\right)
$$

with $h \in I_{1}$. The map $q_{v}$ maps such an annulus isomorphically onto the annulus of radii $\epsilon_{b} / 2$ and $\epsilon_{b}$ around the point $x_{h\left(b^{\prime}\right)} \in \Sigma_{b^{\prime} ; P}$. The key advantage of using the map $\tilde{q}_{v_{0} ; 2}$ instead of $q_{v_{0} ; 2}$ in $(2.18)$ is that

$$
\left\|d \tilde{q}_{v_{0} ; 2}\right\|_{C^{0}\left(\mathcal{A}_{v, h}\right)} \leq C\left|v_{h}\right| \quad \forall h \in I_{1},
$$

where $C^{0}$-norm of $d \tilde{q}_{v_{0} ; 2}$ is computed with respect to the metrics $g_{v_{1}}$ and $g_{v}$ on $\Sigma_{v_{1}}$ and $\Sigma_{v}$, respectively.

Since $\left.u_{v_{1}, \xi}\right|_{\tilde{q}_{v_{0} ; 2}\left(\mathcal{A}_{v, h}\right)}$ is $J_{t}$-holomorphic, (2.7) and (2.8) give

$$
\left\|\left.d u_{v_{1}, \xi}\left|\tilde{q}_{v_{0} ; 2}\left(\mathcal{A}_{v, h}\right) \|_{v_{1}, p} \leq C\right| v_{h}\right|^{2 / p} \sum_{i \in \chi(\mathcal{T}), h(i)=h}\left|\tilde{\rho}_{i}(v)\right| \quad \forall h \in I_{1} ;\right.
$$

see part (2b) of Corollary 3.8 in [Z4]. By (2.28)-(2.30),

$$
\begin{gathered}
\left\|\tilde{\pi}_{v ; \xi}^{t ;+}\left\{\bar{\partial}_{J_{t}}+\nu_{t}\right\} u_{v, \xi}\right\|_{v, p} \leq C \sum_{i \in \chi(\mathcal{T})}\left|\rho_{i}(v)\right| \\
\forall t \in[0, \delta],\left.v \in \widetilde{\mathcal{F}}_{\delta}^{\emptyset}\right|_{W}, \quad \xi \in \Gamma_{t, \delta}(v),
\end{gathered}
$$

if $W$ and $\delta$ are sufficiently small. This estimate is the analogue of the first estimate in part (3) of Lemma 4.4 in $[\mathbf{Z 4}]$. Separately, by the construction of the homomorphism $R_{v, \xi}$,

$$
\operatorname{supp} R_{v, \xi} \eta \cap \operatorname{supp} \tilde{\eta}=\emptyset \quad \forall R_{v, \xi} \eta \in \Gamma_{t ;-}^{0,1}(v ; \xi), \tilde{\eta} \in \tilde{\Gamma}_{t ;-}^{0,1}(v ; \xi) .
$$

Thus, by the $J_{t}$-holomorphicity of $\left.u_{v_{1}, \xi}\right|_{\tilde{q}_{v_{0} ; 2}\left(\mathcal{A}_{v, h}\right)},(2.7),(2.8),(2.28)$, and integration by parts,

$$
\begin{aligned}
& \left|\left\langle\left\langle\left\{\bar{\partial}_{J_{t}}+\nu_{t}\right\} u_{v, \xi}, R_{v, \xi} \eta\right\rangle\right\rangle_{t}+2 \pi \mathfrak{i} \sum_{i \in \chi(\mathcal{T})}\left\langle\mathcal{D}_{i} b^{\prime}, \eta_{x_{h(i)}\left(b^{\prime}\right)}\left(\rho_{i}(v)\right)\right\rangle\right| \\
& \quad \leq C\left(\left\|J_{t}-J_{0}\right\|_{C^{1}}+|v|^{1 / p}+|v|^{(p-2) / p}+\|\xi\|_{v, p, 1}\right)\|\eta\| \sum_{i \in \chi(\mathcal{T})}\left|\rho_{i}(v)\right|
\end{aligned}
$$


see the proof of part (6) of Lemma 4.4 at the end of Subsection 4.2 of $[\mathbf{Z 4}]$. Here $\langle\cdot, \cdot\rangle$ is the Hermitian inner-product on $\left(T_{\operatorname{ev}_{P}\left(b^{\prime}\right)} X, J_{0}\right)$ defined via the metric $g$ on $X$.

We now finish the proof of Proposition 2.7. By (2.22), (2.26), (2.27), (2.31), and the definition of $\Gamma_{+}^{0,1}(v ; \xi)$,

$$
\left\|\zeta_{r}\right\|_{v_{r}, p, 1} \leq C \sum_{i \in \chi(\mathcal{T})}\left|\rho_{i}\left(v_{r}\right)\right|
$$

for all $r$ sufficiently large. Combining this estimate with (2.24), (2.25), and (2.27), we obtain that for all $\eta \in \Gamma_{-}^{0,1}\left(b_{r}^{\prime} ; J_{0}\right)$,

$$
\begin{aligned}
& \left|\left\langle\left\langle D_{v_{r} ; \xi_{r}}^{t_{r}} \zeta_{r}, R_{v_{r}, \xi_{r}} \eta\right\rangle\right\rangle_{t_{r}}\right| \leq C\left|v_{r}\right|^{1 / 2}\|\eta\| \sum_{i \in \chi(\mathcal{T})}\left|\rho_{i}\left(v_{r}\right)\right| ; \\
& \left|\left\langle\left\langle N_{v_{r} ; \xi_{r}}^{t_{r}} \zeta_{r}, R_{v_{r}, \xi_{r}} \eta\right\rangle\right\rangle_{t_{r}}\right| \leq C\left|v_{r}\right|\|\eta\| \sum_{i \in \chi(\mathcal{T})}\left|\rho_{i}\left(v_{r}\right)\right| .
\end{aligned}
$$

Finally, by (2.19), (2.26), (2.32), and (2.34), for a sequence $\epsilon_{r}$ converging to 0

$$
\left|\sum_{i \in \chi(\mathcal{T})}\left\langle\mathcal{D}_{i} b_{r}^{\prime}, \eta_{x_{h(i)}\left(b_{r}^{\prime}\right)}\left(\rho_{i}\left(v_{r}\right)\right)\right\rangle\right| \leq \epsilon_{r}\|\eta\| \sum_{i \in \chi(\mathcal{T})}\left|\rho_{i}\left(v_{r}\right)\right|
$$

for all $\eta \in \mathcal{H}_{b_{r}^{\prime}}^{0,1} \otimes_{J_{0}} T_{\operatorname{ev}_{P}\left(b^{\prime}\right)} X$. Since $v_{r} \in \widetilde{\mathcal{F}}^{\emptyset}$ for all $r, \rho_{i}\left(v_{r}\right) \neq 0$ for all $i \in \chi(b)$. Thus, (2.35) implies the conclusion of Proposition 2.7, since $\mathcal{D}_{i} b_{r}^{\prime} \longrightarrow \mathcal{D}_{i} b$ as $r \longrightarrow \infty$.

\section{Proof of Theorem 1.1}

3.1. Summary. Suppose $(X, \omega), A, k$, and $J$ are as in the statement of Theorem 1.1 and

$$
\psi \in H^{\operatorname{dim}_{1, k}(X, A)}\left(\overline{\mathfrak{M}}_{1, k}(X, A ; J) ; \mathbb{Q}\right)
$$

is a geometric cohomology class. By definition,

$$
\psi=\prod_{l=1}^{l=k} \operatorname{ev}_{l}^{*} \mu_{l} \quad \text { for some } \quad \mu_{l} \in H^{2 n-d_{l}}(X ; \mathbb{Z}), d_{l}<2 n .
$$

For each $l \in[k]$, choose a pseudocycle representative

$$
f_{l}: \bar{Y}_{l} \longrightarrow X
$$

for $\mathrm{PD}_{X} \mu_{l}$. In particular, $\bar{Y}_{l}$ is a disjoint union of smooth manifolds. The dimension of one of them, $Y_{l ; \mathrm{mn}}$, is $d_{l}$; the dimensions of all others are at most $d_{l}-2$. The map $f_{l}$ is continuous, and its restriction to 
each smooth manifold is smooth; see Chapter 7 in $[\mathbf{M c S a}]$ or Section 1 in $[\mathbf{R T}]$. Let

$$
\begin{gathered}
\mathrm{ev}=\prod_{l=1}^{l=k} \mathrm{ev}_{l}: \mathfrak{X}_{1, k}(X, A) \longrightarrow \prod_{l=1}^{l=k} X \\
f=\prod_{l=1}^{l=k} f_{l}: \bar{Y} \equiv \prod_{l=1}^{l=k} \bar{Y}_{l} \longrightarrow \prod_{l=1}^{l=k} X, \quad \text { and } \quad Y_{\mathrm{mn}}=\prod_{l=1}^{l=k} Y_{l ; \mathrm{mn}} .
\end{gathered}
$$

With $\left(f_{l}\right)_{l \in[k]}$ as above, for any $\nu \in \mathfrak{G}_{1, k}^{0,1}(X, A ; J)$ and a bubble type $\mathcal{T}$ as in Subsection 2.2, let

$$
\begin{aligned}
\mathfrak{X}_{1, k}(X, A ; f) & =\left\{(b, z) \in \mathfrak{X}_{1, k}(X, A) \times \bar{Y}: \operatorname{ev}(b)=f(z)\right\}, \\
\overline{\mathfrak{M}}_{1, k}(X, A ; J, \nu ; f) & =\left(\overline{\mathfrak{M}}_{1, k}(X, A ; J, \nu) \times \bar{Y}\right) \cap \mathfrak{X}_{1, k}(X, A ; f), \\
\mathcal{U}_{\mathcal{T}, \nu}(X ; J ; f) & =\left(\mathcal{U}_{\mathcal{T}, \nu}(X ; J) \times \bar{Y}\right) \cap \mathfrak{X}_{1, k}(X, A ; f) .
\end{aligned}
$$

If $\nu$ is sufficiently small, the space $\overline{\mathfrak{M}}_{1, k}(X, A ; J, \nu ; f)$ is compact. Let

$$
\Delta_{X}^{k}=\prod_{l=1}^{l=k} \Delta_{X} \subset \prod_{l=1}^{l=k} X \times X
$$

be the $k$-fold product of the diagonals. If $\nu$ and $f_{l}$ are chosen generically, then

$$
\overline{\mathfrak{M}}_{1, k}(X, A ; J, \nu ; f) \subset \mathfrak{M}_{1, k}^{0}(X, A ; J, \nu) \times Y_{\mathrm{mn}}
$$

and the map

$$
\mathrm{ev} \times f: \mathfrak{M}_{1, k}^{0}(X, A ; J, \nu) \times Y_{\mathrm{mn}} \longrightarrow\left(X^{2}\right)^{k}
$$

is transverse to $\Delta_{X}^{k}$. Thus, $\overline{\mathfrak{M}}_{1, k}(X, A ; J, \nu ; f)$ is a compact zero-dimensional orbifold. By definition,

$$
\mathrm{GW}_{1, k}^{X}(A ; \psi)={ }^{ \pm}\left|\overline{\mathfrak{M}}_{1, k}(X, A ; J, \nu ; f)\right|,
$$

if $\nu$ is sufficiently small and $\nu$ and $f_{l}$ are generic.

Similarly to the previous paragraph, if $\nu_{\mathrm{es}} \in \mathfrak{G}_{1, k}^{\mathrm{es}}(X, A ; J)$, let

$$
\overline{\mathfrak{M}}_{1, k}^{0}\left(X, A ; J, \nu_{\mathrm{es}} ; f\right)=\left(\overline{\mathfrak{M}}_{1, k}^{0}\left(X, A ; J, \nu_{\mathrm{es}}\right) \times \bar{Y}\right) \cap \overline{\mathfrak{M}}_{1, k}\left(X, A ; J, \nu_{\mathrm{es}} ; f\right) \text {. }
$$

For generic $\nu_{\mathrm{es}} \in \mathfrak{G}_{1, k}^{\mathrm{es}}(X, A ; J)$ and $f_{l}$,

$$
\overline{\mathfrak{M}}_{1, k}^{0}\left(X, A ; J, \nu_{\mathrm{es}} ; f\right) \subset \mathfrak{M}_{1, k}^{0}\left(X, A ; J, \nu_{\mathrm{es}}\right) \times Y_{\mathrm{mn}}
$$

and the map

$$
\mathrm{ev} \times f: \mathfrak{M}_{1, k}^{0}(X, A ; J, \nu) \times Y_{\mathrm{mn}} \longrightarrow\left(X^{2}\right)^{k}
$$


is transverse to $\Delta_{X}^{k}$, by the second half of Subsection 1.4. Therefore, $\overline{\mathfrak{M}}_{1, k}^{0}\left(X, A ; J, \nu_{\mathrm{es}} ; f\right)$ is a compact zero-dimensional orbifold. By definition,

$$
\mathrm{GW}_{1, k}^{0 ; X}(A ; \psi)={ }^{ \pm}\left|\overline{\mathfrak{M}}_{1, k}^{0}\left(X, A ; J, \nu_{\mathrm{es}} ; f\right)\right|,
$$

if $\nu_{\mathrm{es}}$ is sufficiently small and $\nu_{\mathrm{es}} \in \mathfrak{G}_{1, k}^{\mathrm{es}}(X, A ; J)$ and $f_{l}$ are generic.

If $\nu_{\mathrm{es}} \in \mathfrak{G}_{1, k}^{\mathrm{es}}(X, A ; J)$ and $f_{l}$ are generic, (3.1) and (3.2) do not generally hold for $\nu=\nu_{\mathrm{es}}$ because the restriction of the bundle section $\bar{\partial}+\nu_{\mathrm{es}}$ is not transverse to the zero set along some strata

$$
\mathfrak{X}_{\mathcal{T}}(X, A) \subset \mathfrak{X}_{1, k}(X, A)-\mathfrak{X}_{1, k}^{\{0\}}(X, A) .
$$

Instead, we will apply (3.1) and (3.2) with $\nu$ replaced by $\nu_{\mathrm{es}}+\nu$ for a generic $\nu \in \mathfrak{G}_{1, k}^{0,1}(X, A ; J)$ which is sufficiently small relatively to $\nu_{\mathrm{es}}$. In such a case, the compact zero-dimensional orbifold

$$
\overline{\mathfrak{M}}_{1, k}\left(X, A ; J, \nu+\nu_{\mathrm{es}} ; f\right)
$$

will lie in a small neighborhood of

$$
\overline{\mathfrak{M}}_{1, k}\left(X, A ; J, \nu_{\mathrm{es}} ; f\right) \subset \mathfrak{X}_{1, k}(X, A ; f) .
$$

We will express the cardinality of this orbifold in terms of data intrinsic to $\overline{\mathfrak{M}}_{1, k}\left(X, A ; J, \nu_{\mathrm{es}} ; f\right)$. From the transversality of the relevant maps, it is straightforward to see that there is a unique element of (3.5) close to each of the elements of $\overline{\mathfrak{M}}_{1, k}^{0}\left(X, A ; J, \nu_{\mathrm{es}}, f\right)$. Thus,

$$
\mathrm{GW}_{1, k}^{X}(A ; \psi)-\mathrm{GW}_{1, k}^{0 ; X}(A ; \psi)
$$

is the number of elements of (3.5) that lie close to the closed subset

$$
\overline{\mathfrak{M}}_{1, k}\left(X, A ; J, \nu_{\mathrm{es}} ; f\right)-\overline{\mathfrak{M}}_{1, k}^{0}\left(X, A ; J, \nu_{\mathrm{es}}\right) \times \bar{Y}
$$

of $\overline{\mathfrak{M}}_{1, k}\left(X, A ; J, \nu_{\mathrm{es}}\right) \times \bar{Y}$. The contribution of (3.6) to (3.4) can in fact be split into contributions of the subspaces $\mathcal{U}_{\mathcal{T}, \nu_{\mathrm{es}}}(X ; J ; f)$ of $(3.6)$. By studying local obstructions similarly to $[\mathbf{Z 1}]$, each of these contributions will be shown to be equal to the number of zeros of an affine bundle map between two finite-rank vector bundles over $\mathcal{U}_{\mathcal{T}, \nu_{\mathrm{es}}}(X ; J ; f)$; see Proposition 3.1 below. Such numbers can be determined using the procedure described in [Z1, Subsections 3.2,3.3].

If $\operatorname{dim}_{\mathbb{R}} X=4$, only two strata of (3.6) are nonempty for a good choice of $\nu_{\mathrm{es}}$. They are isomorphic to

$$
\begin{aligned}
& \mathcal{M}_{1,1} \times \mathfrak{M}_{0,\{0\} \cup[k]}^{0}\left(X, A ; J, \nu_{B} ; f\right) \quad \text { and } \\
& \left(\overline{\mathcal{M}}_{1,1}-\mathcal{M}_{1,1}\right) \times \mathfrak{M}_{0,\{0\} \cup[k]}^{0}\left(X, A ; J, \nu_{B} ; f\right)
\end{aligned}
$$

for some $\nu_{B} \in \mathfrak{G}_{0,\{0\} \cup[k]}^{0,1}(X, A ; J)$, where $\mathcal{M}_{1,1} \subset \overline{\mathcal{M}}_{1,1}$ is the complement of the equivalence class of the singular elliptic curve. The dimension 
of $\mathfrak{M}_{0,\{0\} \cup[k]}^{0}\left(X, A ; J, \nu_{B} ; f\right)$ is zero, even though no constraint has been imposed on the zeroth marked point. Thus, $\mathfrak{M}_{0,\{0\} \cup[k]}^{0}\left(X, A ; J, \nu_{B} ; f\right)$ is "virtually empty". It is thus not too surprising that neither of these strata contributes to $\mathrm{GW}_{1, k}^{X}(A ; \psi)$.

Remark. If $J$ is a genus-one $A$-regular almost complex structure in the sense of $\left[\mathbf{Z 4}\right.$, Definition 1.3], we can take $\nu_{\mathrm{es}}=0$. If $\operatorname{dim}_{\mathbb{R}} X=4$, we then find that the space (3.6) is empty, since

$$
\mathfrak{M}_{0,\{0\} \cup[k]}^{0}(X, A ; J ; f) \equiv \mathfrak{M}_{0,\{0\} \cup[k]}^{0}(X, A ; J, 0 ; f)
$$

cannot be zero-dimensional. Thus, if $(X, \omega)$ admits a genus-one $A$ regular almost complex structure, the first case of Theorem 1.1 is immediate from dimension-counting, once it is known that $\mathrm{GW}_{1, k}^{0 ; X}(A ; \psi)$ is well-defined.

If $\operatorname{dim}_{\mathbb{R}} X=6$, for a good choice of $\nu_{\mathrm{es}}$ only a few strata of (3.6) are nonempty. All, but two of them, are either virtually empty or $\bar{\partial}_{J^{-}}$ hollow, in the sense of [Z1, Subsection 3.1]. In either of these cases, $\mathcal{U}_{\mathcal{T}, \nu_{\text {es }}}(X ; J ; f)$ does not contribute to $(3.2)$. The two remaining strata are isomorphic to

$$
\mathcal{M}_{1,1} \times \mathfrak{M}_{0,\{0\} \cup[k]}^{0}\left(X, A ; J, \nu_{B} ; f\right) \quad \text { and } \quad \mathcal{M}_{1,2} \times \mathfrak{M}_{0, k}^{0}\left(X, A ; J, \nu_{B} ; f\right)
$$

for some $\nu_{B} \in \mathfrak{G}_{0,\{0\} \cup[k]}^{0,1}(X, A ; J)$ or $\nu_{B} \in \mathfrak{G}_{0, k}^{0,1}(X, A ; J)$, respectively. In the second case, one of the elements of $[k]$ corresponds to the attaching node of the only bubble component of each element in $\mathcal{U}_{\mathcal{T}, \nu_{\mathrm{es}}}(X ; J ; f)$; we will denote it by 0 . Let

$$
\begin{aligned}
& L_{1} \longrightarrow \overline{\mathcal{M}}_{1,1}, \overline{\mathcal{M}}_{1,2} \quad \text { and } \\
& L_{0} \longrightarrow \overline{\mathfrak{M}}_{0,\{0\} \sqcup k}\left(X, A ; J, \nu_{B} ; f\right), \mathfrak{M}_{0, k}^{0}\left(X, A ; J, \nu_{B} ; f\right)
\end{aligned}
$$

be the universal tangent line bundles for the marked points labeled by 1 and 0 , respectively. Let

$$
\mathcal{D}_{0}: L_{0} \longrightarrow \mathrm{ev}_{0}^{*} T X
$$

be the vector bundle homomorphism over $\overline{\mathfrak{M}}_{0,\{0\} \sqcup k}\left(X, A ; J, \nu_{B} ; f\right)$ or $\mathfrak{M}_{0, k}^{0}\left(X, A ; J, \nu_{B} ; f\right)$ given by

$$
\mathcal{D}_{0}([b, v])=\left.d u_{b}\right|_{y_{0}(b)} v
$$

We denote by

$$
\begin{aligned}
& \pi_{P}, \pi_{B}: \overline{\mathcal{M}}_{1,1} \times \overline{\mathfrak{M}}_{0,\{0\} \cup[k]}\left(X, A ; J, \nu_{B} ; f\right) \\
& \qquad \overline{\mathcal{M}}_{1,1}, \overline{\mathfrak{M}}_{0,\{0\} \cup[k]}\left(X, A ; J, \nu_{B} ; f\right), \\
& \pi_{P}, \pi_{B}: \overline{\mathcal{M}}_{1,2} \times \mathfrak{M}_{0, k}^{0}\left(X, A ; J, \nu_{B} ; f\right) \longrightarrow \overline{\mathcal{M}}_{1,2}, \mathfrak{M}_{0, k}^{0}\left(X, A ; J, \nu_{B} ; f\right)
\end{aligned}
$$


the two projection maps. In both cases, the linear part of the affine map determining the contribution of the stratum $\mathcal{U}_{\mathcal{T}, \nu_{\mathrm{es}}}(X ; J ; f)$ to $(3.2)$ is

$$
\begin{gathered}
\mathcal{D}_{\mathcal{T}}: \pi_{P}^{*} L_{1} \otimes \pi_{B}^{*} L_{0} \longrightarrow \pi_{P}^{*} \mathbb{E}^{*} \otimes \pi_{B}^{*} \operatorname{ev}_{0}^{*} T X \\
\left\{\mathcal{D}_{\mathcal{T}}\left[b_{P}, b_{B}, v_{P} \otimes v_{B}\right]\right\}\left(\left[b_{B}, \psi\right]\right)=\psi_{x_{1}\left(b_{P}\right)}\left(v_{P}\right) \cdot{ }_{J} \mathcal{D}_{0}\left(\left[b_{B}, v_{B}\right]\right), \\
\text { if } \quad\left[b_{P}, b_{B}, v_{P} \otimes v_{B}\right] \in \pi_{P}^{*} L_{1} \otimes \pi_{B}^{*} L_{0},\left[b_{B}, \psi\right] \in \pi_{P}^{*} \mathbb{E} .
\end{gathered}
$$

The constant term $\bar{\nu}$ of each of the affine maps is generic. In the second case, $\mathfrak{M}_{0, k}^{0}\left(X, A ; J, \nu_{B} ; f\right)$ is a finite collection of points. It is then straightforward to see that for a generic $\nu$, the affine bundle map $\mathcal{D}_{\mathcal{T}}+\bar{\nu}$ does not vanish. Thus, the corresponding stratum $\mathcal{U}_{\mathcal{T}, \nu_{\mathrm{es}}}(X ; J ; f)$ does not contribute to (3.2). We will show in Subsection 3.4 that the number $N\left(\mathcal{D}_{\mathcal{T}}\right)$ of zeros of $\mathcal{D}_{\mathcal{T}}+\bar{\nu}$ in the first case is

$$
N\left(\mathcal{D}_{\mathcal{T}}\right)=\frac{2-\left\langle c_{1}(T X), A\right\rangle}{24} \mathrm{GW}_{0, k}^{X}(A ; \psi),
$$

proving the second case of Theorem 1.1.

Remark. If $J$ is a genus-one $A$-regular almost complex structure and $\operatorname{dim}_{\mathbb{R}} X=6$, the space (3.6) is the union of the spaces

$$
\overline{\mathfrak{M}}_{1, k}\left(\kappa, 1 ;\left.J\right|_{\kappa} ;\left.\underline{f}\right|_{\kappa}\right)
$$

taken over all degree- $A$ genus-zero curves $\kappa$ in $X$ that intersect $f_{l}\left(Y_{l ; \mathrm{mn}}\right)$ for every $l \in[k]$. Based on $[\mathbf{P a}]$, one would expect that each of the spaces (3.7) contributes $\left(2-\left\langle c_{1}(T X), A\right\rangle\right) / 24$ to $\mathrm{GW}_{1, k}^{X}(A ; \psi)$. The total number of the curves $\kappa$ is $\mathrm{GW}_{0, k}^{X}(A ; \psi)$. In particular, the second case of Theorem 1.1, just like the first, is consistent with geometric expectations.

3.2. Analytic Setup. Let $\mathcal{T}=([k], I, \aleph ; j, \underline{A})$ be a bubble type such that

$$
A_{i}=0 \forall i \in I_{0} \quad \text { and } \quad \sum_{i \in I} A_{i}=A .
$$

For each $i \in I-I_{0}$, let

$$
H_{i} \mathcal{T}=\left\{h \in \hat{I}: \iota_{h}=i\right\} \quad \text { and } \quad M_{i} \mathcal{T}=\left\{l \in[k]: j_{l}=i\right\} .
$$

We denote by

$$
\pi_{\mathcal{T} ; i}: \mathfrak{X}_{\mathcal{T}}(X) \longrightarrow \mathfrak{X}_{0,\{0\} \sqcup H_{i} \mathcal{T} \sqcup M_{i} \mathcal{T}}\left(X, A_{i}\right)
$$

the map sending each element $[b]$ of $\mathfrak{X}_{\mathcal{T}}(X)$ to its restriction to $\Sigma_{b, i}$ :

$$
\begin{aligned}
{[(S,[k], I, \aleph ;} & x,(j, y), u)] \\
& \longrightarrow\left[\left(H_{i} \mathcal{T} \sqcup M_{i} \mathcal{T},\{i\} ;,\left.\left.(\iota, x)\right|_{H_{i}} \mathcal{T} \sqcup(j, y)\right|_{M_{i} \mathcal{T}}, u_{i}\right)\right] .
\end{aligned}
$$


Let $\mathfrak{G}_{1, k}^{\mathrm{gd}}(X, A ; J)$ be the subspace of elements $\nu$ in $\mathfrak{G}_{1, k}^{\text {es }}(X, A ; J)$ such that for every bubble type $\mathcal{T}$ as above,

$$
\left.\nu\right|_{\mathfrak{X}_{\mathcal{T}}(X)}=\sum_{i \in I-I_{0}} \pi_{\mathcal{T} ; i}^{*} \nu_{\mathcal{T} ; i} \quad \text { for some } \quad \nu_{\mathcal{T} ; i} \in \mathfrak{G}_{0,\{0\} \sqcup H_{i} \mathcal{T} \sqcup M_{i} \mathcal{T}}^{0,1}\left(X, A_{i} ; J\right)
$$

and for every $[b] \in \mathfrak{M}_{0,\{0\} \sqcup H_{i} \mathcal{T} \sqcup M_{i} \mathcal{T}}^{0}\left(X, A_{i} ; J, \nu_{\mathcal{T} ; i}\right)$ the linearization

$$
\begin{aligned}
D_{J, \nu_{\mathcal{T}} ; i} ; & \left\{\xi \in \Gamma\left(\Sigma_{b} ; u_{b}^{*} T X\right): \xi\left(y_{0}(b)\right)=0\right\} \\
& \longrightarrow \Gamma\left(\Sigma_{b} ; \Lambda_{J, j}^{0,1} T^{*} \Sigma_{b} \otimes u_{b}^{*} T X\right)
\end{aligned}
$$

of the section $\bar{\partial}_{J}+\nu_{\mathcal{T} ; i}$ at $b$ is surjective.

For a generic element $\nu_{\mathcal{T} ; i} \in \mathfrak{G}_{0,\{0\} \sqcup H_{i}}^{\mathrm{es}} \mathcal{T}_{M_{i}} \mathcal{T}\left(X, A_{i} ; J\right)$, the operator (3.9) is surjective for every

$$
[b] \in \overline{\mathfrak{M}}_{0,\{0\} \sqcup H_{i} \mathcal{T} \sqcup M_{i} \mathcal{T}}\left(X, A_{i} ; J, \nu_{\mathcal{T} ; i}\right) .
$$

This implies that the closure of $\mathfrak{G}_{1, k}^{\text {gd }}(X, A ; J)$ in $\mathfrak{G}_{1, k}^{0,1}(X, A ; J)$ contains the zero section, since we can construct an element $\nu$ of $\mathfrak{G}_{1, k}^{\mathrm{gd}}(X, A ; J)$ inductively starting from the highest-codimension strata of $\mathfrak{X}_{1, k}(X, A)$. If $\mathcal{T}$ is a bubble type as above and $\nu$ has been defined on

$$
\overline{\mathfrak{X}}_{\mathcal{T}}(X)-\mathfrak{X}_{\mathcal{T}}(X) \subset \mathfrak{X}_{1, k}(X, A)
$$

subject to the above restriction and regularity conditions, then $\nu$ induces a multisection $\nu_{\mathcal{T} ; i}$ of

$$
\begin{aligned}
\Gamma_{0,\{0\} \sqcup H_{i} \mathcal{T} \sqcup M_{i} \mathcal{T}}^{0,1} & \left(X, A_{i} ; J\right) \longrightarrow \\
& \mathfrak{X}_{0,\{0\} \sqcup H_{i} \mathcal{T} \sqcup M_{i} \mathcal{T}}\left(X, A_{i}\right)-\mathfrak{X}_{0,\{0\} \sqcup H_{i} \mathcal{T} \sqcup M_{i} \mathcal{T}}^{0}\left(X, A_{i}\right) .
\end{aligned}
$$

It extends continuously to an effectively supported multisection over all of $\mathfrak{X}_{0,\{0\} \sqcup H_{i} \mathcal{T} \sqcup M_{i} \mathcal{T}}\left(X, A_{i}\right)$. By perturbing this extension outside of

$$
\mathfrak{X}_{0,\{0\} \sqcup H_{i} \mathcal{T} \sqcup M_{i} \mathcal{T}}\left(X, A_{i}\right)-\mathfrak{X}_{0,\{0\} \sqcup H_{i} \mathcal{T} \sqcup M_{i} \mathcal{T}}^{0}\left(X, A_{i}\right),
$$

we obtain an element $\nu_{\mathcal{T} ; i}$ of $\mathfrak{G}_{0,\{0\} \sqcup H_{i} \mathcal{T} \sqcup M_{i} \mathcal{T}}\left(X, A_{i} ; J\right)$ such that the operator (3.9) is surjective for every

$$
[b] \in \overline{\mathfrak{M}}_{0,\{0\} \sqcup H_{i} \mathcal{T} \sqcup M_{i} \mathcal{T}}\left(X, A_{i} ; J, \nu_{\mathcal{T} ; i}\right) .
$$

We fix small generic elements

$$
\nu_{\mathrm{es}} \in \mathfrak{G}_{1, k}^{\mathrm{gd}}(X, A ; J) \quad \text { and } \quad \nu \in \mathfrak{G}_{1, k}^{0,1}(X, A ; J)
$$

such that for all $t \in \mathbb{R}^{+}$sufficiently small the section

$$
\left.\left\{\bar{\partial}_{J}+\nu_{\mathrm{es}}+t \nu\right\}\right|_{\mathfrak{X}_{\mathcal{T}}(X)}
$$


is transverse to the zero set in $\left.\Gamma_{1, k}^{0,1}(X, A ; J)\right|_{\mathfrak{X}_{\mathcal{T}}(X)}$ for every stratum $\mathfrak{X}_{\mathcal{T}}(X)$ of $\mathfrak{X}_{1, k}(X, A)$. Let $\left\{Y_{\lambda}\right\}_{\lambda \in \mathcal{A}}$ be the strata of $\bar{Y}$ induced by the partitions of each $\bar{Y}_{l}$ into smooth manifolds. By our assumptions,

$$
\begin{aligned}
\operatorname{dim}_{\mathbb{R}} Y_{\mathrm{mn}} & =2 n k-\operatorname{dim}_{1, k}(X, A), \\
\operatorname{dim}_{\mathbb{R}} Y_{\lambda} & \leq \operatorname{dim}_{\mathbb{R}} Y_{\mathrm{mn}}-2 \forall \lambda \in \mathcal{A}-\{0\} .
\end{aligned}
$$

For each bubble type $\mathcal{T}$ as above and $\lambda \in \mathcal{A}$, let

$$
\mathcal{U}_{\mathcal{T}, \nu_{\mathrm{es}}}\left(X ; J ; f_{\lambda}\right)=\left(\mathcal{U}_{\mathcal{T}, \nu_{\mathrm{es}}}(X ; J) \times Y_{\lambda}\right) \cap \overline{\mathfrak{M}}_{1, k}\left(X, A ; J, \nu_{\mathrm{es}} ; f\right) .
$$

We will call a bubble type $\mathcal{T}$ as above simple if

$$
\aleph=\emptyset \quad \text { and } \quad \hat{I}=\chi(\mathcal{T}) .
$$

In other words, $\mathcal{T}$ is simple if and only if for every element $[b]$ of $\mathcal{U}_{\mathcal{T}, \nu_{\text {es }}}(X, A ; J)$ the domain $\Sigma_{b}$ consists of a smooth principal component $\Sigma_{b ; P}$, on which the map $u_{b}$ is necessarily constant, and $|\hat{I}|$ bubble components, all of which are attached directly to $\Sigma_{b ; P}$ and on which the map $u_{b}$ is not constant.

Suppose $\overline{\mathcal{M}}$ is a compact topological space which is a disjoint union of smooth orbifolds, one of which, $\mathcal{M}$, is a dense open subset of $\overline{\mathcal{M}}$, and the dimensions of all others do not exceed $\operatorname{dim} \mathcal{M}-2$. Let

$$
E, \mathcal{O} \longrightarrow \overline{\mathcal{M}}
$$

be vector bundles such that the restrictions of $E$ and $\mathcal{O}$ to every stratum of $\overline{\mathcal{M}}$ is smooth and

$$
\operatorname{rk} \mathcal{O}-\operatorname{rk} E=\frac{1}{2} \operatorname{dim}_{\mathbb{R}} \mathcal{M}
$$

If

$$
\alpha \in \Gamma(\overline{\mathcal{M}} ; \operatorname{Hom}(E, \mathcal{O}))
$$

is a regular section in the sense of [Z1, Definition 3.9], then the cardinality of the zero set of the affine bundle map

$$
\psi_{\alpha, \bar{\nu}} \equiv \alpha+\bar{\nu}: E \longrightarrow \mathcal{O}
$$

is finite and independent of a generic choice of $\bar{\nu} \in \Gamma(\overline{\mathcal{M}} ; \mathcal{O})$, by $[\mathbf{Z 1}$, Lemma 3.14]. We denote it by $N(\alpha)$. A key step in our proof of Theorem 1.1 is the following proposition.

Proposition 3.1. Suppose $(X, \omega, J), A, k, \psi, f_{l}, \nu_{\mathrm{es}}, \nu$, and $\left\{Y_{\lambda}\right\}_{\lambda \in \mathcal{A}}$ are as above. If $\mathcal{T}$ is a bubble as above and $\lambda \in \mathcal{A}$, there exist

$$
\mathcal{C}_{\mathcal{U}_{\mathcal{T}, \nu_{\mathrm{es}}}\left(X ; J ; f_{\lambda}\right)}\left(\bar{\partial}_{J}\right) \in \mathbb{Q}, \quad \epsilon_{\nu} \in \mathbb{R}^{+},
$$

and a compact subset $K_{\nu}$ of $\mathcal{U}_{\mathcal{T}, \nu_{\mathrm{es}}}\left(X ; J ; f_{\lambda}\right)$ with the following property. For every compact subset $K$ of $\mathcal{U}_{\mathcal{T}, \nu_{\mathrm{es}}}\left(X ; J ; f_{\lambda}\right)$ and open subset 
$U$ of $\mathfrak{X}_{1, k}(X, A ; f)$, there exist an open neighborhood $U_{\nu}(K)$ of $K$ in $\mathfrak{X}_{1, k}(X, A ; f)$ and $\epsilon_{\nu}(U) \in\left(0, \epsilon_{\nu}\right)$, respectively, such that

$$
{ }^{ \pm}\left|\overline{\mathfrak{M}}_{1, k}\left(X, A ; J, \nu_{\mathrm{es}}+t \nu ; f\right) \cap U\right|=\mathcal{C}_{\mathcal{U}_{\mathcal{T}, \nu_{\mathrm{es}}}\left(X ; J ; f_{\lambda}\right)}\left(\bar{\partial}_{J}\right)
$$

if $t \in\left(0, \epsilon_{\nu}(U)\right), K_{\nu} \subset K \subset U \subset U_{\nu}(K)$. Furthermore, if $\mathcal{T}$ is simple and $\lambda=\mathrm{mn}$,

$$
\mathcal{C}_{\mathcal{U}_{\mathcal{T}, \nu_{\mathrm{es}}}\left(X ; J ; f_{\lambda}\right)}\left(\bar{\partial}_{J}\right)=N\left(\mathcal{D}_{\mathcal{T}}\right)
$$

for some regular vector bundle homomorphism $\mathcal{D}_{T}$ over $\mathcal{U}_{\mathcal{T}, \nu_{\mathrm{es}}}(X ; J ; f)$. Otherwise,

$$
\mathcal{C}_{\mathcal{U}_{\mathcal{T}, \nu \text { es }}\left(X ; J ; f_{\lambda}\right)}\left(\bar{\partial}_{J}\right)=0
$$

This proposition is proved in the next subsection. In the previous subsection we described the homomorphism $\mathcal{D}_{\mathcal{T}}$ for a simple bubble type $\mathcal{T}$ such that $|\chi(\mathcal{T})|=1$. Below we describe this homomorphism for an arbitrary bubble $\mathcal{T}$ satisfying (3.8).

For each $i \in \hat{I}$, let

$$
\tilde{H}_{i} \mathcal{T}=\left\{h \in \hat{I}: \iota_{h} \geq i\right\}, \quad \tilde{M}_{i} \mathcal{T}=\left\{l \in[k]: j_{l} \geq i\right\}, \quad \tilde{A}_{i}=\sum_{h \geq i} A_{h} .
$$

We denote by

$$
\tilde{\pi}_{\mathcal{T} ; i}: \mathfrak{X}_{\mathcal{T}}(X) \longrightarrow \mathfrak{X}_{0,\{0\} \sqcup \tilde{M}_{i} \mathcal{T}}\left(X, \tilde{A}_{i}\right)
$$

the map sending each element $[b]$ of $\mathfrak{X}_{\mathcal{T}}(X)$ to its restriction to the tree of bubble components beginning with $\Sigma_{b, i}$ :

$$
\begin{aligned}
& {[(S,[k], I, \aleph ; x,(j, y), u)]} \\
& \qquad\left[\left(\tilde{M}_{i} \mathcal{T},\{i\} \cup \tilde{H}_{i} \mathcal{T} ;\left.(\iota, x)\right|_{\tilde{H}_{i} \mathcal{T}},\left.(j, y)\right|_{\tilde{M}_{i} \mathcal{T}},\left.u\right|_{\{i\} \cup \tilde{H}_{i} \mathcal{T}}\right)\right] .
\end{aligned}
$$

By our assumptions on $\nu_{\mathrm{es}}$,

$$
\tilde{\pi}_{\mathcal{T} ; i}: \mathcal{U}_{\mathcal{T}, \nu_{\mathrm{es}}}(X ; J) \longrightarrow \overline{\mathfrak{M}}_{0,\{0\} \sqcup \tilde{M}_{i} \mathcal{T}}\left(X, \tilde{A}_{i} ; J, \tilde{\nu}_{B ; i}\right)
$$

for some $\tilde{\nu}_{B ; i} \in \mathfrak{G}_{0,\{0\} \sqcup \tilde{M}_{i} \mathcal{T}}^{\text {es }}\left(X, \tilde{A}_{i} ; J\right)$. Let

$$
\mathfrak{F} \mathcal{T}=\left(\bigoplus_{i \in \chi(\mathcal{T})} \pi_{P}^{*} L_{h(i)} \otimes \tilde{\pi}_{\mathcal{T} ; i}^{*} L_{0}\right) / \operatorname{Aut}(\mathcal{T}) \rightarrow \mathcal{U}_{\mathcal{T}, \nu_{\mathrm{es}}}(X ; J), \mathcal{U}_{\mathcal{T}, \nu_{\mathrm{es}}}(X ; J ; f)
$$

If $M_{0}$ is a finite set and $h \in M_{0}$, let

$$
s_{h} \in \Gamma\left(\overline{\mathcal{M}}_{1, M_{0}} ; \operatorname{Hom}\left(L_{h}, \mathbb{E}^{*}\right)\right)
$$

be the section given by

$$
\left\{s_{h}(b ; v)\right\}(\psi)=\psi_{x_{h}(b)} v \in \mathbb{C} \quad \text { if }\left.\quad v \in L_{h}\right|_{b}, \psi \in \mathbb{E}_{b},
$$

where $x_{h}(b) \in \Sigma_{b}$ is the $h$-th marked point. We define the homomorphism

$$
\mathcal{D}_{\mathcal{T}}: \mathfrak{F} \mathcal{T} \longrightarrow\left(\pi_{P}^{*} \mathbb{E}^{*} \otimes \operatorname{ev}_{P}^{*} T X\right) / \operatorname{Aut}(\mathcal{T})
$$


over $\mathcal{U}_{\mathcal{T}, \nu_{\mathrm{es}}}(X ; J)$ or $\mathcal{U}_{\mathcal{T}, \nu_{\mathrm{es}}}(X ; J ; f)$ by

$$
\mathcal{D}_{\mathcal{T}}=\sum_{i \in \chi(\mathcal{T})} \pi_{P}^{*} s_{h(i)} \otimes \tilde{\pi}_{\mathcal{T} ; i}^{*} \mathcal{D}_{0}
$$

3.3. Proof of Proposition 3.1. We continue with the notation of Subsections 2.4 and 3.2. By our assumptions on $\nu_{\mathrm{es}}$, the operators $D_{J, \nu_{\mathrm{es}} ; b, i}$ are surjective for all $[b] \in \mathcal{U}_{\mathcal{T}, \nu_{\mathrm{es}}}(X ; J)$ and $i \in \hat{I}$. Thus, we can take

$$
\tilde{\Gamma}_{-}^{0,1}(b ; i)=\{0\} \quad \forall[b] \in \mathcal{U}_{\mathcal{T}, \nu_{\mathrm{es}}}(X ; J), i \in I .
$$

The corresponding space $\tilde{\mathcal{U}}$ of Subsection 2.4 is a smooth manifold of $\left(J, \nu_{\mathrm{es}}\right)$-holomorphic maps. In Subsections 2.5 of $[\mathbf{Z 2}]$ and 4.2 of [Z4], we describe a space $\mathcal{U}_{\mathcal{T}}^{(0)}(X ; J)$ of balanced $J$-holomorphic maps, not of equivalence classes of such maps. If $\nu_{\mathrm{es}}$ is sufficiently small, the same definitions can be used to describe a submanifold $\mathcal{U}_{\mathcal{T}, \nu_{\text {es }}}^{(0)}(X ; J)$ of $\tilde{\mathcal{U}}$. In particular,

$$
\mathcal{U}_{\mathcal{T}, \nu_{\mathrm{es}}}(X ; J)=\mathcal{U}_{\mathcal{T}, \nu_{\mathrm{es}}}^{(0)}(X ; J) / \operatorname{Aut}(\mathcal{T}) \propto\left(S^{1}\right)^{\hat{I}}
$$

for a natural action of $\operatorname{Aut}(\mathcal{T})$ on $\left(S^{1}\right)^{\hat{I}}$ and $\operatorname{of} \operatorname{Aut}(\mathcal{T}) \propto\left(S^{1}\right)^{\hat{I}}$ on $\tilde{\mathcal{U}}$. Let

$$
\tilde{\mathcal{F}} \mathcal{T}=\left.\widetilde{\mathcal{F}}\right|_{\mathcal{U}_{\mathcal{T}, \nu_{\mathrm{es}}}^{(0)}(X ; J)},
$$

where $\widetilde{\mathcal{F}} \tilde{\mathcal{U}}$ is the vector bundle defined in Subsection 2.5. The above group action on $\tilde{\mathcal{U}}$ lifts to an action on $\widetilde{\mathcal{F}}$ so that

$$
\mathcal{F} \mathcal{T} \equiv \tilde{\mathcal{F}} \mathcal{T} / \operatorname{Aut}(\mathcal{T}) \propto\left(S^{1}\right)^{\hat{I}}
$$

is the bundle of gluing parameters for $\mathcal{U}_{\mathcal{T}, \nu_{\mathrm{es}}}(X ; J)$.

We will apply the construction of Subsection 2.5, with some refinements, to the entire space $\mathcal{U}_{\mathcal{T}}^{(0)}, \nu_{\text {es }}(X ; J)$, instead of a small open subset of $\tilde{\mathcal{U}}$. We will view $\mathbb{R}$-valued functions on $\mathcal{U}_{\mathcal{T}, \nu_{\mathrm{es}}}(X ; J)$ as functions on $\mathcal{U}_{\mathcal{T}, \nu_{\mathrm{es}}}^{(0)}(X ; J)$ via the quotient projection map

$$
\mathcal{U}_{\mathcal{T}, \nu_{\mathrm{es}}}^{(0)}(X ; J) \longrightarrow \mathcal{U}_{\mathcal{T}, \nu_{\mathrm{es}}}(X ; J) .
$$

Fix small

$$
\delta, \epsilon \in C^{\infty}\left(\mathcal{U}_{\mathcal{T}, \nu_{\mathrm{es}}}(X ; J) ; \mathbb{R}^{+}\right)
$$

such that the basic gluing map

$$
q_{v}: \Sigma_{v} \longrightarrow \Sigma_{b}
$$

of [Z2, Subsection 2.2] and the modified gluing map

$$
\tilde{q}_{v_{0} ; 2}: \Sigma_{v} \longrightarrow \Sigma_{v_{1}}
$$


of [ $\mathbf{Z 4}$, Subsection 4.2] with the collapsing radius $\epsilon(b)$ are defined for all $v \equiv(b, v) \in \tilde{\mathcal{F}} \mathcal{T}_{\delta}$. For all

$$
\xi \in \Gamma\left(v_{1}\right) \quad \text { s.t. } \quad\|\xi\|_{v_{1}, p, 1} \leq \delta(b),
$$

let $u_{v_{1}, \xi}, u_{v, \xi}, \Gamma_{+}(v ; \xi)$,

$$
D_{J, \nu_{\mathrm{es}} ; v ; \xi}: \Gamma(v ; \xi) \longrightarrow \Gamma^{0,1}(v ; \xi), \quad R_{v, \xi}: \Gamma_{-}^{0,1}(b ; J) \longrightarrow \Gamma^{0,1}(v ; \xi),
$$

and $\nu_{\mathrm{es} ; v_{1}, \xi}$ be as in Subsection 2.5, with $J_{t}, \nu_{t}$, and $\nabla^{t}$ replaced by $J, \nu_{\mathrm{es}}$, and a $J$-compatible connection $\nabla$, respectively. The estimates $(2.24)$, (2.25), (2.27), and (2.29)-(2.32) continue to hold if $C \in \mathbb{R}^{+}, D_{v ; \xi}^{t}, J_{t}$, and $\nu_{t}$ are replaced by

$$
C \in C^{\infty}\left(\mathcal{U}_{\mathcal{T}, \nu_{\mathrm{es}}}(X ; J) ; \mathbb{R}^{+}\right),
$$

$D_{J, \nu_{\mathrm{es}} ; v ; \xi}, J$, and $\nu_{\mathrm{es}}$, respectively. In $(2.31)$ and $(2.32), \tilde{\pi}_{v ; \xi}^{t ;+}=\mathrm{id}$ and $J_{0}=J$.

With notation as in Subsection 2.5, for each $v \in \tilde{\mathcal{F}} \mathcal{T}_{\delta}^{\emptyset}$ let

$$
\Gamma_{B ;-}\left(v_{1}\right)=\left\{\xi \circ q_{v_{1}}: \xi \in \operatorname{ker} D_{J, \nu_{\mathrm{es}} ; b},\left.\xi\right|_{\Sigma_{b ; P}}=0\right\} .
$$

We denote by $\Gamma_{B ;+}\left(v_{1}\right)$ the $L^{2}$-orthogonal complement of $\Gamma_{B ;-}\left(v_{1}\right)$ in $\Gamma_{B}\left(v_{1}\right)$. Let

$$
D_{J, \nu_{\mathrm{es}} ; v_{1}}^{B}: \Gamma_{B}\left(v_{1}\right) \longrightarrow \Gamma_{B}^{0,1}\left(v_{1}\right)
$$

be the linearization of section $\bar{\partial}_{J}+\nu_{\text {es }}$ at $\left(\Sigma_{v_{1}} ; u_{v_{1}}\right)$ defined via the connection $\nabla$. Similarly to $(2.22)$,

$$
\begin{gathered}
C(b)^{-1}\|\xi\|_{v_{1}, p, 1} \leq\left\|D_{J, \nu_{\mathrm{es}} ; v_{1}}^{B} \xi\right\|_{v_{1}, p} \leq C(b)\|\xi\|_{v_{1}, p, 1} \\
\forall v=(b, v) \in \tilde{\mathcal{F}} \mathcal{T}_{\delta}^{\emptyset}, \quad \xi \in \Gamma_{B ;+}\left(v_{1}\right),
\end{gathered}
$$

for some $C \in C^{\infty}\left(\mathcal{U}_{\mathcal{T}, \nu_{\text {es }}}(X ; J) ; \mathbb{R}^{+}\right)$, provided $\delta \in C^{\infty}\left(\mathcal{U}_{\mathcal{T}, \nu_{\text {es }}}(X ; J) ; \mathbb{R}^{+}\right)$ is sufficiently small. In particular, the operator

$$
D_{J, \nu_{\mathrm{es}} ; v_{1}}^{B}: \Gamma_{B ;+}\left(v_{1}\right) \longrightarrow \Gamma_{B}^{0,1}\left(v_{1}\right)
$$

is an isomorphism. Its norm and the norm of its inverse are dependent only on $[b] \in \mathcal{U}_{\mathcal{T} ; \nu_{\text {es }}}(X ; J)$. Thus, by the Contraction Principle, for each $v \in \tilde{\mathcal{F}} \mathcal{T}_{\delta}^{\emptyset}$, the equation

$$
\left\{\bar{\partial}_{J}+\nu_{\mathrm{es} ; v_{1}, \xi}\right\} u_{v_{1}, \xi}=0, \quad \xi \in \Gamma_{B ;+}\left(v_{1}\right),
$$

has a unique small solution $\xi_{\nu_{\mathrm{es}}}\left(v_{1}\right)$.

Remark. Since $\nu_{\mathrm{es}}$ is a multisection, the uniqueness statement above, as well as similar statements below, should be interpreted in terms of local branches of $\nu_{\mathrm{es}}$ as defined in [FuOn, Section 3]. 
Lemma 3.2. If $\mathcal{T}$ is a bubble type as in (3.8) and $\nu_{\mathrm{es}} \in \mathfrak{G}_{1, k}^{\mathrm{gd}}(X, A ; J)$ is a sufficiently small generic perturbation, there exist

$$
\delta \in C^{\infty}\left(\mathcal{U}_{\mathcal{T}, \nu_{\mathrm{es}}}(X ; J) ; \mathbb{R}^{+}\right)
$$

and an open neighborhood $U_{\mathcal{T}}$ of $\mathcal{U}_{\mathcal{T}, \nu_{\mathrm{es}}}(X ; J)$ in $\mathfrak{X}_{1, k}(X, A)$ such that the map

$$
\begin{aligned}
& \left\{(v, \zeta): v=(b, v) \in \tilde{\mathcal{F}} \mathcal{T}_{\delta}^{\emptyset} ; \zeta \in \Gamma_{+}\left(v ; \xi_{\nu_{\mathrm{es}}}\left(v_{1}\right)\right),\right. \\
& \left.\|\zeta\|_{v, p, 1}<\delta(b)\right\} / \operatorname{Aut}(\mathcal{T}) \propto\left(S^{1}\right)^{|\hat{I}|} \longrightarrow \mathfrak{X}_{1, k}^{0}(X, A) \cap U_{\mathcal{T}},
\end{aligned}
$$

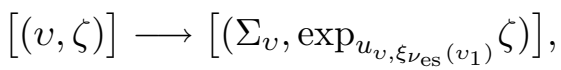

is a diffeomorphism.

Proof. It is immediate from the construction that the map

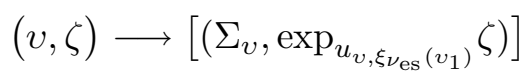

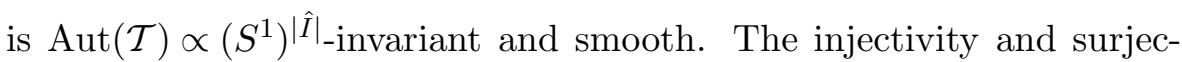
tivity of the induced map on the quotient are proved by arguments similar to Subsections 4.2 and 4.3-4.5 in [Z2], respectively; see also the paragraph following Lemma 4.4 in $[\mathbf{Z 4}]$.

q.e.d.

For each $v=(b, v) \in \tilde{\mathcal{F}} \mathcal{T}_{\delta}^{\emptyset}$, we define the homomorphism

$$
\pi_{v ;-}^{0,1}: \Gamma^{0,1}\left(v ; \xi_{\nu_{\mathrm{es}}}\left(v_{1}\right)\right) \longrightarrow \Gamma_{-}^{0,1}(b ; J) \approx \mathbb{E}_{\pi_{P}(b)}^{*} \otimes T_{\mathrm{ev}_{P}(b)} X
$$

as follows. If $\left\{\eta_{r}\right\}_{r \in[n]}$ is an orthonormal basis for $\Gamma_{-}^{0,1}(b ; J)$, we put

$$
\pi_{v ;-}^{0,1} \eta^{\prime}=\sum_{r=1}^{r=n}\left\langle\left\langle\eta^{\prime}, R_{v, \xi_{\nu \mathrm{es}}\left(v_{1}\right)} \eta_{r}\right\rangle\right\rangle \eta_{r} \quad \forall \eta^{\prime} \in \Gamma^{0,1}\left(v ; \xi_{\nu_{\mathrm{es}}}\left(v_{1}\right)\right) .
$$

This map is well-defined.

For each $(v, \zeta)$ as above, let

$$
\begin{aligned}
& \Pi_{\zeta}: L^{p}\left(\Sigma_{v} ; \Lambda_{J, j}^{0,1} T^{*} \Sigma_{v} \otimes u_{v, \xi_{\nu_{\mathrm{es}}}\left(v_{1}\right)} T X\right)
\end{aligned}
$$

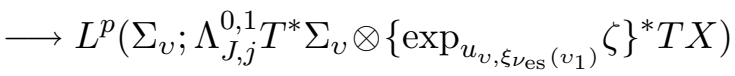

be the isomorphism induced by the $\nabla$-parallel transport along the $\nabla$ geodesics

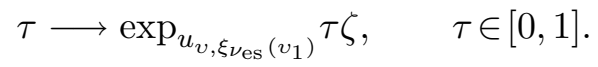

Similarly to $(2.26)$,

$$
\begin{aligned}
& \Phi_{t}(v, \zeta) \equiv \Pi_{\zeta}^{-1}\left\{\bar{\partial}_{J}+\nu_{\mathrm{es}}+t \nu\right\} \exp _{u_{v, \xi_{\nu \mathrm{es}}\left(v_{1}\right)} \zeta} \\
& =\left\{\bar{\partial}_{J}+\nu_{\mathrm{es}}\right\} u_{v}+D_{J, \nu_{\mathrm{es}} ; v ; \xi_{\nu_{\mathrm{es}}}\left(v_{1}\right)} \zeta+t \nu\left(\Sigma_{v}, u_{v, \xi_{\nu \mathrm{es}}\left(v_{1}\right)}\right)+N_{v ; t} \xi \text {, }
\end{aligned}
$$


where the quadratic term $N_{v ; t}$ satisfies

$$
\begin{aligned}
N_{v ; t} 0 & =0 \\
\left\|N_{v ; t} \zeta-N_{v ; t} \zeta^{\prime}\right\|_{v, p} & \leq C(b)\left(t+\|\zeta\|_{v, p, 1}+\left\|\zeta^{\prime}\right\|_{v, p, 1}\right)\left\|\zeta-\zeta^{\prime}\right\|_{v, p, 1}
\end{aligned}
$$

whenever $t \in[0, \delta(b)], v=(b, v) \in \tilde{\mathcal{F}} \mathcal{T}_{\delta}^{\emptyset}$, and

$$
\zeta, \zeta^{\prime} \in \Gamma\left(v ; \xi_{\nu_{\mathrm{es}}}\left(v_{1}\right)\right) \text { s.t. }\|\zeta\|_{v, p, 1},\left\|\zeta^{\prime}\right\|_{v, p, 1} \leq \delta(b) .
$$

Thus, by Lemma 3.2, the analogues of (2.22) and (2.31) mentioned above, for every precompact open subset $K$ of $\mathcal{U}_{\mathcal{T}, \nu}(X ; A)$ there exist $\delta_{K}, C_{K} \in \mathbb{R}^{+}$and a neighborhood of $U_{K}$ of $K$ in $\mathfrak{X}_{1, k}(X, A)$ such that for all $t \in\left[0, \delta_{K}\right]$

$$
\mathfrak{M}_{1, k}^{0}\left(X, A ; J, \nu_{\mathrm{es}}+t \nu\right) \cap U_{K} \approx\left\{[(v, \zeta)] \in \Omega_{K}(t): \Phi_{t}(v, \zeta)=0\right\},
$$

where

$$
\begin{aligned}
\Omega_{K}(t)=\{[(v, \zeta)]: v & =\left.(b, v) \in \tilde{\mathcal{F}} \mathcal{T}_{\delta_{K}}^{\emptyset}\right|_{K} ; \\
\zeta & \left.\in \Gamma_{+}\left(v ; \xi_{\nu_{\mathrm{es}}}\left(v_{1}\right)\right),\|\zeta\|_{v, p, 1} \leq C_{K}(|\rho(v)|+t)\right\} .
\end{aligned}
$$

For each $b \in \mathcal{U}_{\mathcal{T}, \nu_{\mathrm{es}}}^{(0)}(X ; J)$, let

$$
\bar{\nu}_{b} \in \Gamma_{-}^{0,1}(b ; J)
$$

be the $L^{2}$-projection of $\nu(b)$. We note that the map

$$
\mathfrak{G}_{1, k}^{0,1}(X, A ; J) \longrightarrow \Gamma\left(\mathcal{U}_{\mathcal{T}, \nu_{\mathrm{es}}}(X ; J) ; \pi_{P}^{*} \mathbb{E}^{*} \otimes \operatorname{ev}_{P}^{*} T X\right), \quad \nu \longrightarrow \bar{\nu},
$$

is surjective for every bubble type $\mathcal{T}$. By (3.11), (3.12), and the analogues of (2.24), (2.25), and (2.32),

$$
\begin{gathered}
\left\|\pi_{v ;-}^{0,1} \Phi_{t}(v, \zeta)-\left(\mathcal{D}_{\mathcal{T}} \rho(v)+t \bar{\nu}_{b}\right)\right\| \leq \varepsilon(v)(|\rho(v)|+t) \\
\forall \quad t \in\left[0, \delta_{K}\right], t \in\left[0, \delta_{K}\right], v=\left.(b, v) \in \tilde{\mathcal{F}} \mathcal{T}_{\delta_{K}}^{\emptyset}\right|_{K},[(v, \zeta)] \in \Omega_{K}(t),
\end{gathered}
$$

for some function

$$
\varepsilon: \tilde{\mathcal{F}} \mathcal{T}^{\emptyset} \longrightarrow \mathbb{R}^{+} \quad \text { s.t. } \quad \lim _{|v| \longrightarrow 0} \varepsilon(v)=0 .
$$

We denote by

$$
\pi_{\mathbb{P} \mathfrak{F}}: \mathbb{P} \mathfrak{F} \mathcal{T} \longrightarrow \mathcal{U}_{\mathcal{T}, \nu_{\mathrm{es}}}(X ; J) \quad \text { and } \quad \gamma_{\mathbb{P} \mathfrak{F}} \longrightarrow \mathbb{P} \mathfrak{F} \mathcal{T}
$$

the bundle projection map and the tautological line bundle. If $\nu_{\mathrm{es}} \in$ $\mathfrak{G}_{1, k}^{\mathrm{gd}}(X, A ; J)$ is generic, the section

$$
\tilde{\mathcal{D}}_{\mathcal{T}} \in \Gamma\left(\mathcal{U}_{\mathcal{T}, \nu_{\mathrm{es}}}(X ; J) ; \operatorname{Hom}\left(\gamma_{\mathbb{P} \mathfrak{F} \mathcal{T}} ; \pi_{\mathbb{P} \mathfrak{T} \mathcal{T}}^{*}\left(\pi_{P}^{*} \mathbb{E}^{*} \otimes \operatorname{ev}_{P}^{*} T X\right)\right)\right.
$$


induced by $\mathcal{D}_{\mathcal{T}}$ is transverse to the zero set. For a generic choice of the pseudocycles $f_{l}$, this is also the case for the restriction of $\tilde{\mathcal{D}}_{\mathcal{T}}$ to $\left.\mathbb{P} \mathfrak{F}\right|_{\mathcal{U}_{\mathcal{T}, \nu_{\text {es }}}\left(X ; J ; f_{\lambda}\right)}$ for every $\lambda \in \mathcal{A}$. On the other hand,

$$
\begin{aligned}
\operatorname{dim} & \mathcal{U}_{\mathcal{T}, \nu_{\mathrm{es}}}\left(X ; J ; f_{\lambda}\right)=\operatorname{dim} \mathcal{U}_{\mathcal{T}, \nu_{\mathrm{es}}}(X ; J)+\left(\operatorname{dim} Y_{\lambda}-n k\right) \\
& \leq\left(\operatorname{dim}_{1, k}(X, A)+2(n-|\hat{I}|-|\aleph|)\right)-\operatorname{dim}_{1, k}(X, A) \\
& =2(n-|\chi(\mathcal{T})|)-2(|\hat{I}-\chi(\mathcal{T})|+|\aleph|) .
\end{aligned}
$$

The middle inequality is an equality if and only if $\lambda=\mathrm{mn}$. Thus, the section $\tilde{\mathcal{D}}_{\mathcal{T}}$ does not vanish over $\left.\mathbb{P} \mathfrak{F} \mathcal{T}\right|_{\mathcal{U}_{\mathcal{T}, \nu_{\text {es }}}\left(X ; J ; f_{\lambda}\right)}$. This is equivalent to saying that the bundle homomorphism

$$
\mathcal{D}_{\mathcal{T}}: \mathfrak{F} \mathcal{T} \longrightarrow \pi_{P}^{*} \mathbb{E}^{*} \otimes \mathrm{ev}_{P}^{*} T X
$$

is nondegenerate over $\mathcal{U}_{\mathcal{T}, \nu_{\text {es }}}\left(X ; J ; f_{\lambda}\right)$, i.e. is injective on every fiber over $\mathcal{U}_{\mathcal{T}, \nu_{\mathrm{es}}}\left(X ; J ; f_{\lambda}\right)$.

Suppose $\mathcal{T}$ is not a simple bubble type or $\lambda \neq$ mn. By (3.15),

$$
\frac{1}{2} \operatorname{dim} \mathcal{U}_{\mathcal{T}, \nu_{\mathrm{es}}}\left(X ; J ; f_{\lambda}\right)+\operatorname{rk} \mathfrak{F} \mathcal{T}<\operatorname{rk} \pi_{P}^{*} \mathbb{E}^{*} \otimes \mathrm{ev}_{P}^{*} T X .
$$

Thus, for a generic $\nu \in \mathfrak{G}_{1, k}^{\text {gd }}(X, A ; J)$, the affine bundle map

$$
\mathfrak{F} \mathcal{T} \longrightarrow \pi_{P}^{*} \mathbb{E}^{*} \otimes \mathrm{ev}_{P}^{*} T X, \quad v=[b, v] \longrightarrow \mathcal{D}_{\mathcal{T}} v+\bar{\nu}_{b},
$$

has no zeros over $\mathcal{U}_{\mathcal{T}, \nu_{\mathrm{es}}}\left(X ; J ; f_{\lambda}\right)$. Since $\mathcal{D}_{\mathcal{T}}$ is nondegenerate over $\mathcal{U}_{\mathcal{T}, \nu_{\mathrm{es}}}\left(X ; J ; f_{\lambda}\right),(3.14)$ and the proof of Lemma 3.2 in $[\mathbf{Z 1}]$ then imply that for every compact subset $K$ of $\mathcal{U}_{\mathcal{T}, \nu_{\text {es }}}\left(X ; J ; f_{\lambda}\right)$ there exist $\delta_{K} \in \mathbb{R}^{+}$ and a neighborhood $U_{K}^{\prime}$ of $K$ in $\mathcal{U}_{\mathcal{T}, \nu_{\text {es }}}(X ; J) \times \bar{Y}$ such that

$$
\left\{\left.[(v, \zeta), z] \in \Omega_{K}(t)\right|_{U_{K}^{\prime}}: \pi_{v ;-}^{0,1} \Phi_{t}(v, \zeta)=0\right\}=\emptyset \quad \forall t \in\left(0, \delta_{K}\right) .
$$

Thus, there exists a neighborhood $U_{K}$ of $K$ in $\mathfrak{X}_{1, k}(X, A) \times \bar{Y}$ such that

$$
\left(\mathfrak{M}_{1, k}^{0}\left(X, A ; J, \nu_{\mathrm{es}}+t \nu\right) \times \bar{Y}\right) \cap U_{K}=\emptyset .
$$

The proof of Proposition 3.1 in the case $\mathcal{T}$ is not a simple bubble type or $\lambda \neq \mathrm{mn}$ is now complete.

For remainder of this subsection we assume that $\mathcal{T}$ is a simple bubble type and $\lambda=\mathrm{mn}$. If $v=(b, v) \in \tilde{\mathcal{F}} \mathcal{T}_{\delta}^{\emptyset}$, we denote by

$$
\Gamma_{-}^{0,1}(v) \subset \Gamma^{0,1}\left(v ; \xi_{\nu_{\mathrm{es}}}\left(v_{1}\right)\right)
$$

the image of $\Gamma_{-}^{0,1}(b ; J)$ under $R_{v}$ and by $\Gamma_{+}^{0,1}(v)$ the $L^{2}$-orthogonal complement of $\Gamma_{-}^{0,1}(v)$ in $\Gamma^{0,1}\left(v ; \xi_{\nu_{\mathrm{es}}}\left(v_{1}\right)\right)$. Let

$$
\pi_{v ;+}^{0,1}: \Gamma^{0,1}\left(v ; \xi_{\nu_{\mathrm{es}}}\left(v_{1}\right)\right) \longrightarrow \Gamma_{+}^{0,1}(v)
$$

the $L^{2}$-projection map. Since $\aleph=\emptyset$,

$$
\left\|R_{v, \xi_{\nu \mathrm{es}}\left(v_{1}\right)} \eta\right\|_{v, p} \leq C(b)\left\|R_{v, \xi_{\nu \mathrm{es}}\left(v_{1}\right)} \eta\right\|_{v, 2} \forall v=(b, v) \in \tilde{\mathcal{F}} \mathcal{T}_{\delta}^{\emptyset}, \eta \in \Gamma_{-}^{0,1}(b ; J),
$$


for some $C \in C^{\infty}\left(\mathcal{U}_{\mathcal{T}, \nu_{\text {es }}}(X ; J) ; \mathbb{R}^{+}\right)$. It follows that

$$
\left\|\pi_{+}^{0,1} \eta^{\prime}\right\|_{v, p} \leq C(b)\left\|\eta^{\prime}\right\|_{v, p} \quad \forall v=(b, v) \in \tilde{\mathcal{F}} \mathcal{T}_{\delta}^{\emptyset}, \eta^{\prime} \in \Gamma^{0,1}\left(v ; \xi_{\nu_{\mathrm{es}}}\left(v_{1}\right)\right) .
$$

Thus, by the analogues of (2.22) and (2.25),

$$
\begin{gathered}
C(b)^{-1}\|\zeta\|_{v, p, 1} \leq\left\|\pi_{+}^{0,1} D_{J, \nu_{\mathrm{es}} ; v ; \xi_{\nu_{\mathrm{es}}}\left(v_{1}\right)} \zeta\right\|_{v, p} \leq C(b)\|\zeta\|_{v, p, 1} \\
\forall \quad v=(b, v) \in \tilde{\mathcal{F}} \mathcal{T}_{\delta}^{\emptyset}, \quad \zeta \in \Gamma_{+}\left(v ; \xi_{\nu_{\mathrm{es}}}\left(v_{1}\right)\right) .
\end{gathered}
$$

In particular, the operator

$$
\pi_{+}^{0,1} D_{J, \nu_{\mathrm{es}} ; v ; \xi_{\nu_{\mathrm{es}}}\left(v_{1}\right)}: \Gamma_{+}\left(v ; \xi_{\nu_{\mathrm{es}}}\left(v_{1}\right)\right) \longrightarrow \Gamma_{+}^{0,1}(v)
$$

is an isomorphism. Its norm and the norm of its inverse are dependent only on $[b] \in \mathcal{U}_{\mathcal{T}, \nu_{\mathrm{es}}}(X ; J)$. By (3.11), (3.12), (3.16), the analogue of (2.31), and the Contraction Principle, for every compact subset $K$ of $\mathcal{U}_{\mathcal{T}, \nu_{\text {es }}}(X ; J)$ there exists $\delta_{K} \in \mathbb{R}^{+}$such that for all

$$
\left.v \in \tilde{\mathcal{F}} \mathcal{T}_{\delta_{K}}^{\emptyset}\right|_{K} \quad \text { and } \quad t \in\left[0, \delta_{K}\right]
$$

the equation

$$
\pi_{+}^{0,1} \Phi_{t}(v, \zeta)=0, \quad \zeta \in \Gamma_{+}\left(v ; \xi_{\nu_{\mathrm{es}}}\left(v_{1}\right)\right)
$$

has a unique small solution $\zeta_{t}(v)$. Furthermore, $\zeta_{t}(v) \in \Omega_{K}(t)$.

By the above, for every compact subset $K$ of $\mathcal{U}_{\mathcal{T}, \nu_{\mathrm{es}}}\left(X ; J ; f_{\mathrm{mn}}\right)$ there exist $\delta_{K} \in \mathbb{R}^{+}$and small neighborhoods $U_{K}^{\prime}$ and $U_{K}$ of $K$ in $\mathcal{U}_{\mathcal{T}}, \nu_{\text {es }}(X ; J) \times$ $Y_{\mathrm{mn}}$ and $\mathfrak{X}_{1, k}(X, A) \times \bar{Y}$, respectively, such that

$$
\begin{aligned}
& \mathfrak{M}_{1, k}^{0}\left(X, A ; J, \nu_{\mathrm{es}}+t \nu ; f_{\mathrm{mn}}\right) \cap U_{K} \\
& \approx\left\{\left.([v], z) \in \mathcal{F} \mathcal{T}_{\delta_{K}}^{\emptyset}\right|_{K} \times Y_{\mathrm{mn}}: \pi_{v ;-}^{0,1} \Phi_{t}\left(v, \zeta_{t}(v)\right)=0 ;\right. \\
& \quad\{\operatorname{ev} \times f\}\left(\left(\Sigma_{v}, \exp _{\left.\left.\left.u_{v, \xi_{\nu_{\mathrm{e}}}\left(v_{1}\right)} \zeta_{t}(v)\right), z\right) \in \Delta_{X}^{k}\right\}}\right.\right.
\end{aligned}
$$

for all $t \in\left[0, \delta_{K}\right]$. On the other hand, the bundle homomorphism $\mathcal{D}_{\mathcal{T}}$ is regular over $\overline{\mathcal{U}}_{\mathcal{T}, \nu_{\text {es }}}(X ; J ; f)$ by the $m=1$ case of $(2.7)$ and $(2.8)$, i.e. $\mathcal{D}_{\mathcal{T}}$ can be approximated by a polynomial on the normal bundle near every stratum of $\overline{\mathcal{U}}_{\mathcal{T}, \nu_{\text {es }}}(X ; J ; f)$; see $[\mathbf{Z} \mathbf{1}$, Definition 3.9]. Since $\nu$ is generic, $\mathcal{D}_{\mathcal{T}}$ is nondegenerate over $\mathcal{U}_{\mathcal{T}, \nu_{\mathrm{es}}}\left(X ; J ; f_{\mathrm{mn}}\right)$, and ev $\times f$ is transverse to $\Delta_{X}^{k}$ in $\left(X^{2}\right)^{k},(3.13)$ and the proof of Lemma 3.5 in $[\mathbf{Z 1}]$ then imply that there exists a compact subset $K_{\nu}$ of $\mathcal{U}_{\mathcal{T}, \nu_{\mathrm{es}}}\left(X ; J ; f_{\mathrm{mn}}\right)$ with the following property. For every compact subset $K$ of $\mathcal{U}_{\mathcal{T}, \nu_{\mathrm{es}}}\left(X ; J ; f_{\mathrm{mn}}\right)$ that contains $K_{\nu}$ there exist $\delta_{K} \in \mathbb{R}^{+}$and a neighborhood $U_{K}$ of $K$ in $\mathfrak{X}_{1, k}(X, A) \times \bar{Y}$ such that

$$
{ }^{ \pm}\left|\mathfrak{M}_{1, k}^{0}\left(X, A ; J, \nu_{\mathrm{es}}+t \nu ; f_{\mathrm{mn}}\right) \cap U_{K}\right|=N\left(\mathcal{D}_{\mathcal{T}}\right) \quad \forall t \in\left[0, \delta_{K}\right] .
$$

The set $K_{\nu}$ can be taken to be any compact subset of $\mathcal{U}_{\mathcal{T}, \nu_{\mathrm{es}}}\left(X ; J ; f_{\mathrm{mn}}\right)$ such that all of the finitely many zeros of the affine map

$$
\mathcal{D}_{\mathcal{T}}+\bar{\nu}: \mathfrak{F} \mathcal{T} \longrightarrow \pi_{P}^{*} \mathbb{E}^{*} \otimes \operatorname{ev}_{P}^{*} T X
$$


over $\mathcal{U}_{\mathcal{T}, \nu_{\text {es }}}\left(X ; J ; f_{\text {mn }}\right)$ lie in $\left.\mathfrak{F} \mathcal{T}\right|_{K_{\nu}}$.

3.4. Counting Zeros of Affine Bundle Maps. In this subsection we conclude the proof of Theorem 1.1 by computing the numbers $N\left(\mathcal{D}_{\mathcal{T}}\right)$ when the dimension of $X$ is 4 or 6 . Using the method of $[\mathbf{Z 1}$, Section 3], $N\left(\mathcal{D}_{\mathcal{T}}\right)$ can be computed for arbitrary-dimensional symplectic manifolds $X$ and more general cohomology classes $\psi$; this is the main subject of $[\mathbf{Z 6}]$. In order to avoid introducing quite a bit of additional notation in this paper we restrict the computation to the special cases of Theorem 1.1.

If $\mathcal{T}$ is a bubble type as in (3.8), let

$$
\begin{aligned}
M_{P} \mathcal{T} & =\left\{l \in[k]: j_{l} \notin \hat{I}\right\}, \\
\operatorname{Aut}^{*}(\mathcal{T}) & =\operatorname{Aut}(\mathcal{T}) /\left\{g \in \operatorname{Aut}(\mathcal{T}): g \cdot h=h \forall h \in I_{1}\right\} .
\end{aligned}
$$

For each $i \in I_{1}$, let

$$
\tilde{\mathcal{T}}_{i}=\left(\tilde{M}_{i} \mathcal{T},\{i\} \cup \tilde{H}_{i} \mathcal{T} ;\left.j\right|_{\tilde{M}_{i} \mathcal{T}},\left.\underline{A}\right|_{\{i\} \cup \tilde{H}_{i} \mathcal{T}}\right) .
$$

If $\nu_{\mathrm{es}} \in \mathfrak{G}_{1, k}^{\mathrm{gd}}(X, A ; J)$ and $\aleph=\emptyset$, then

$$
\mathcal{U}_{\mathcal{T}, \nu_{\mathrm{es}}}(X ; J) \approx\left(\mathcal{M}_{1, M_{P}} \mathcal{T} \sqcup I_{1} \times \mathcal{U}_{\overline{\mathcal{T}}, \nu_{\mathrm{es}}}(X ; J)\right) / \operatorname{Aut}^{*}(\mathcal{T})
$$

where $\mathcal{M}_{1, M_{P}} \mathcal{T} \sqcup I_{1}$ is the subspace of the moduli space $\overline{\mathcal{M}}_{1, M_{P}} \mathcal{T} \sqcup I_{1}$ consisting of smooth curves and

$$
\begin{aligned}
\mathcal{U}_{\overline{\mathcal{T}}, \nu_{\mathrm{es}}}(X ; J)=\left\{\left(b_{i}\right)_{i \in I_{1}} \in \prod_{i \in I_{1}} \mathcal{U}_{\mathcal{T}_{i}, \nu_{B ; i}}:\right. & \\
& \left.\quad \operatorname{ev}_{0}\left(b_{i_{1}}\right)=\operatorname{ev}_{0}\left(b_{i_{2}}\right) \forall i_{1}, i_{2} \in I_{1}\right\},
\end{aligned}
$$

for some $\nu_{B ; i} \in \mathfrak{G}_{0,\{0\} \cup \tilde{M}_{i} \mathcal{T}}^{\text {es }}\left(X, \tilde{A}_{i} ; J\right)$; in particular,

$$
\mathcal{U}_{\overline{\mathcal{T}}, \nu_{\mathrm{es}}}(X ; J) \subset \prod_{i \in I_{1}} \overline{\mathfrak{M}}_{0,\{0\} \cup \tilde{M}_{i} \mathcal{T}}\left(X, \tilde{A}_{i} ; J, \nu_{B ; i}\right) .
$$

This decomposition is illustrated in Figure 5. In this figure, we represent an entire stratum $\mathcal{U}_{\mathcal{T}, \nu_{\mathrm{es}}}(X ; J)$ of bubble maps by the domain of the stable maps in $\mathcal{U}_{\mathcal{T}, \nu_{\mathrm{es}}}(X ; J)$. The right-hand side of Figure 5 represents the subset of the cartesian product of the three spaces of bubble maps, corresponding to the three drawings, on which the appropriate evaluation maps agree, as indicated by the solid line and defined in (3.18).

We next define a similar splitting for the space $\mathcal{U}_{\mathcal{T}, \nu_{\mathrm{es}}}\left(X ; J ; f_{\mathrm{mn}}\right)$. We can assume that the maps $\left\{f_{l ; m n}\right\}_{l \in M_{P} \mathcal{T}}$ intersect transversally. Let

$$
f_{0}^{\mathcal{T}}: \bar{Y}_{0}^{\mathcal{T}} \longrightarrow \bigcap_{l \in M_{P} \mathcal{T}} f_{l}\left(\bar{Y}_{l}\right) \subset X
$$



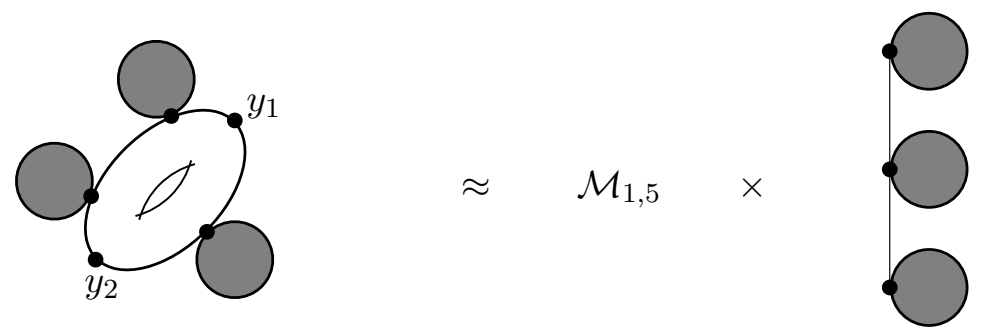

Figure 5. An example of the decomposition (3.17).

be a pseudocycle representative for

$$
\bigcap_{l \in M_{P} \mathcal{T}} \mathrm{PD}_{X} \psi_{l} \in H_{*}(X ; \mathbb{Z})
$$

such that

$$
f_{0 ; \mathrm{mn}}^{\mathcal{T}}: Y_{0 ; \mathrm{mn}}^{\mathcal{T}} \longrightarrow \bigcap_{l \in M_{P} \mathcal{T}} f_{l}\left(\bar{Y}_{l ; \mathrm{mn}}\right)
$$

is one-to-one. We put

$$
\begin{aligned}
\mathrm{ev}^{\mathcal{T}} & =\mathrm{ev}_{0} \times \prod_{l \in[k]-M_{P} \mathcal{T}} \mathrm{ev}_{l}: \mathcal{U}_{\overline{\mathcal{T}}, \nu_{\mathrm{es}}}(X ; J) \longrightarrow X \times \prod_{l \in[k]-M_{P} \mathcal{T}} X, \\
f^{\mathcal{T}} & =f_{0}^{\mathcal{T}} \times \prod_{l \in[k]-M_{P} \mathcal{T}} f_{l}: \bar{Y}^{\mathcal{T}} \equiv \bar{Y}_{0}^{\mathcal{T}} \times \prod_{l \in[k]-M_{P} \mathcal{T}} \bar{Y}_{l} \longrightarrow X \times \prod_{l \in[k]-M_{P} \mathcal{T}} X, \\
Y_{\mathrm{mn}}^{\mathcal{T}} & =Y_{0 ; \mathrm{mn}}^{\mathcal{T}} \times \prod_{l \in[k]-M_{P} \mathcal{T}} Y_{l ; \mathrm{mn}} .
\end{aligned}
$$

Similarly to Subsection 3.1, let

$$
\begin{aligned}
\overline{\mathcal{U}}_{\overline{\mathcal{T}}, \nu}(X ; J ; f) & =\left\{(b, z) \in \overline{\mathcal{U}}_{\overline{\mathcal{T}}, \nu}(X ; J) \times \bar{Y}^{\mathcal{T}}: \mathrm{ev}^{\mathcal{T}}(b)=f^{\mathcal{T}}(z)\right\}, \\
\mathcal{U}_{\overline{\mathcal{T}}, \nu}\left(X ; J ; f_{\mathrm{mn}}\right) & =\left(\mathcal{U}_{\overline{\mathcal{T}}, \nu}(X ; J) \times Y_{\mathrm{mn}}^{\mathcal{T}}\right) \cap \overline{\mathcal{U}}_{\overline{\mathcal{T}}, \nu}(X ; J ; f) .
\end{aligned}
$$

We have

$$
\mathcal{U}_{\mathcal{T}, \nu_{\mathrm{es}}}\left(X ; J ; f_{\mathrm{mn}}\right) \approx\left(\mathcal{M}_{1, M_{P}} \mathcal{T} \sqcup I_{1} \times \mathcal{U}_{\overline{\mathcal{T}}, \nu_{\mathrm{es}}}\left(X ; J ; f_{\mathrm{mn}}\right)\right) / \operatorname{Aut}^{*}(\mathcal{T})
$$

If $\mathcal{T}$ is a simple bubble type, we define the homomorphism

$$
\widetilde{\mathcal{D}}_{\mathcal{T}}: \widetilde{\mathfrak{F}} \mathcal{T} \longrightarrow \pi_{P}^{*} \mathbb{E}^{*} \otimes \mathrm{ev}_{0}^{*} T X
$$

over $\overline{\mathcal{M}}_{1, M_{P}} \mathcal{T} \sqcup I_{1} \times \overline{\mathcal{U}}_{\overline{\mathcal{T}}, \nu_{\text {es }}}(X ; J ; f)$ similarly to the homomorphism $\mathcal{D}_{\mathcal{T}}$. By (3.19),

$$
N\left(\mathcal{D}_{\mathcal{T}}\right)=N\left(\widetilde{\mathcal{D}}_{\mathcal{T}}\right) /\left|\operatorname{Aut}^{*}(\mathcal{T})\right| .
$$

By Proposition 3.1 and (3.20), the difference between the standard and reduced genus-one GW-invariants of Theorem 1.1 is determined by the numbers $N\left(\widetilde{\mathcal{D}}_{\mathcal{T}}\right)$, where $\mathcal{T}$ is a simple bubble type as in (3.8). We will 
compute these numbers in the two special cases of Theorem 1.1.

First, we note that if $\mathcal{T}$ is a bubble type as in (3.8), not necessarily simple, and $\aleph=\emptyset$,

$$
\begin{gathered}
\operatorname{dim} \overline{\mathcal{U}}_{\overline{\mathcal{T}}, \nu_{\mathrm{es}}}(X ; J ; f)=\operatorname{dim} \overline{\mathcal{U}}_{\mathcal{T}, \nu_{\mathrm{es}}}(X ; J ; f)-\operatorname{dim} \overline{\mathcal{M}}_{1, M_{P} \mathcal{T} \sqcup I_{1}} \\
=2\left(n-|\chi(\mathcal{T})|-\left|I_{1}\right|\right)-2\left(|\hat{I}-\chi(\mathcal{T})|+\left|M_{P} \mathcal{T}\right|\right),
\end{gathered}
$$

by (3.15). In particular, if $n=2$, then

$$
\overline{\mathcal{U}}_{\overline{\mathcal{T}}, \nu_{\mathrm{es}}}(X ; J ; f) \neq \emptyset \quad \Longrightarrow \quad \chi(\mathcal{T}) \mid=1, \chi(\mathcal{T})=I_{1}=\hat{I}, M_{P} \mathcal{T}=\emptyset
$$

Furthermore, if $\overline{\mathcal{U}}_{\overline{\mathcal{T}}, \nu_{\mathrm{es}}}(X ; J ; f)$ is nonempty, it is a finite collection of points. In this case, $\widetilde{\mathcal{D}}_{\mathcal{T}}$ is the bundle homomorphism

$$
\pi_{P}^{*}\left(s_{1} \oplus 0\right): \pi_{P}^{*} L_{1} \longrightarrow \pi_{P}^{*}\left(\mathbb{E}^{*} \oplus \mathbb{E}^{*}\right)
$$

over $\overline{\mathcal{M}}_{1,1} \times \overline{\mathcal{U}}_{\overline{\mathcal{T}}, \nu_{\mathrm{es}}}(X ; J ; f)$. Thus,

$$
N\left(\widetilde{\mathcal{D}}_{\mathcal{T}}\right)=-\frac{1}{24}{ }^{ \pm}\left|\overline{\mathcal{U}}_{\overline{\mathcal{T}}, \nu_{\mathrm{es}}}(X ; J ; f)\right| .
$$

Since ${ }^{ \pm}\left|\overline{\mathcal{U}}_{\overline{\mathcal{T}}, \nu_{\text {es }}}(X ; J ; f)\right|={ }^{ \pm}\left|\overline{\mathcal{U}}_{\overline{\mathcal{T}}, \nu^{\prime}}(X ; J ; f)\right|$ for any sufficiently small $\nu^{\prime}$ such that the restriction of the bundle section $\bar{\partial}+\nu^{\prime}$ to every stratum of $\mathfrak{X}_{0,\{0\} \sqcup[k]}(X, A)$ is transverse to the zero set, we can take $\nu^{\prime}=\pi^{*} \nu_{0}$, where

$$
\pi: \mathfrak{X}_{0,\{0\} \sqcup[k]}(X, A) \longrightarrow \mathfrak{X}_{0,[k]}(X, A)
$$

is the forgetful map and $\nu_{0}$ is a small generic deformation of $\bar{\partial}$ on $\mathfrak{X}_{0,[k]}(X, A)$. Since

$$
\overline{\mathfrak{M}}_{0, k}\left(X, A ; J, \nu_{0} ; f\right)=\emptyset
$$

for a generic $\nu_{0}$ for dimensional reasons, it follows $\overline{\mathcal{U}}_{\overline{\mathcal{T}}, \nu^{\prime}}(X ; J ; f)=\emptyset$ as well and thus $N\left(\widetilde{\mathcal{D}}_{\mathcal{T}}\right)=0$, as claimed by $n=2$ case of Theorem 1.1.

Suppose $n=3$. By $(3.21)$, if $\overline{\mathcal{U}}_{\overline{\mathcal{T}}, \nu_{\mathrm{es}}}(X ; J ; f) \neq \emptyset$, then

$$
|\chi(\mathcal{T})|=1, \quad\left(\left|I_{1}\right|-|\chi(\mathcal{T})|\right)+|\hat{I}-\chi(\mathcal{T})|+\left|M_{P} \mathcal{T}\right| \in\{0,1\} .
$$

If $\mathcal{T}$ is a simple bubble type and $\overline{\mathcal{U}}_{\overline{\mathcal{T}}, \nu_{\text {es }}}(X ; J ; f)$ is nonempty, it follows that

$$
|\chi(\mathcal{T})|=1 \quad \text { and } \quad\left|M_{P} \mathcal{T}\right| \in\{0,1\} .
$$

If $\left|M_{P} \mathcal{T}\right|=1, \overline{\mathcal{U}}_{\overline{\mathcal{T}}, \nu_{\text {es }}}(X ; J ; f)$ is again a finite collection of points. In this case, $\widetilde{\mathcal{D}}_{\mathcal{T}}$ is the bundle homomorphism

$$
\pi_{P}^{*}\left(s_{1} \oplus 0 \oplus 0\right): \pi_{P}^{*} L_{1} \longrightarrow \pi_{P}^{*}\left(\mathbb{E}^{*} \oplus \mathbb{E}^{*} \oplus \mathbb{E}^{*}\right)
$$

over $\overline{\mathcal{M}}_{1,2} \times \overline{\mathcal{U}}_{\overline{\mathcal{T}}, \nu_{\mathrm{es}}}(X ; J ; f)$. As in the $n=2$ case, ${ }^{ \pm}\left|\overline{\mathcal{U}}_{\overline{\mathcal{T}}, \nu_{\mathrm{es}}}(X ; J ; f)\right|=0$. Furthermore, since $c_{1}\left(\mathbb{E}^{*}\right)^{2}=0$ on $\overline{\mathcal{M}}_{1,2}$, we can choose $\nu$ so that $\bar{\nu}$ does not vanish and thus $N\left(\widetilde{\mathcal{D}}_{\mathcal{T}}\right)=0$ by definition. Thus, $N\left(\widetilde{\mathcal{D}}_{\mathcal{T}}\right)=0$. 
Finally, suppose $n=3, \mathcal{T}$ is a simple bubble type, and $\left|M_{P} \mathcal{T}\right|=0$. If $i$ is the unique element of $\chi(\mathcal{T}), \widetilde{\mathcal{D}}_{\mathcal{T}}$ is the bundle homomorphism

$$
\pi_{P}^{*} s_{1} \otimes \pi_{i}^{*} \mathcal{D}_{0}: \pi_{P}^{*} L_{1} \otimes \pi_{i}^{*} L_{0} \longrightarrow \pi_{P}^{*} \mathbb{E}^{*} \otimes \pi_{i}^{*} \mathrm{ev}_{0}^{*} T X
$$

over

$$
\overline{\mathcal{M}}_{1,1} \times \overline{\mathcal{U}}_{\overline{\mathcal{T}}, \nu_{\mathrm{es}}}(X ; J ; f)=\overline{\mathcal{M}}_{1,1} \times \overline{\mathfrak{M}}_{0,\{0\} \cup[k]}\left(X, A ; J, \nu_{B ; i} ; f\right) .
$$

We assume that $\nu_{B, i}=\pi^{*} \nu_{B}$ for a generic element $\nu_{B} \in \mathfrak{G}_{0,\{0\}}^{\text {es }}(X, A ; J)$, where

$$
\pi: \mathfrak{X}_{0,\{0\} \sqcup[k]}(X, A) \longrightarrow \mathfrak{X}_{0,\{0\}}(X, A)
$$

is the forgetful map. Then,

$$
\pi: \overline{\mathfrak{M}}_{0,\{0\} \cup[k]}\left(X, A ; J, \nu_{B ; i} ; f\right) \longrightarrow \overline{\mathfrak{M}}_{0,\{0\}}\left(X, A ; J, \nu_{B}\right)
$$

and there are identifications $L_{0}=\pi^{*} L_{0}$ and $\mathcal{D}_{0}=\pi^{*} \mathcal{D}_{0}$. Thus,

$$
N\left(\widetilde{\mathcal{D}}_{\mathcal{T}}\right)=N\left(\pi_{P}^{*} s_{1} \otimes \pi_{i}^{*} \pi^{*} \mathcal{D}_{0}\right) .
$$

On the other hand, if $\nu_{B}$ is generic, the image of the projection $\pi$ in (3.22) is contained in $\mathfrak{M}_{0,\{0\}}^{\{0\}}\left(X, A ; J, \nu_{B}\right)$, while the restriction of $\mathcal{D}_{0}$ to every stratum of $\mathfrak{M}_{0,\{0\}}^{\{0\}}\left(X, A ; J, \nu_{B}\right)$ is transverse to the zero set. Thus, $\pi^{*} \mathcal{D}_{0}$ does not vanish over $\overline{\mathfrak{M}}_{0,\{0\} \cup[k]}\left(X, A ; J, \nu_{B ; i} ; f\right)$ for dimensional reasons if $\nu_{B}$ is generic. Since $s_{1}$ does not vanish over $\overline{\mathcal{M}}_{1,1}$, the bundle homomorphism

$$
\pi_{P}^{*} s_{1} \otimes \pi_{i}^{*} \pi^{*} \mathcal{D}_{0}: \pi_{P}^{*} L_{1} \otimes \pi_{i}^{*} \pi^{*} L_{0} \longrightarrow \pi_{P}^{*} \mathbb{E}^{*} \otimes \pi_{i}^{*} \pi^{*} \mathrm{ev}_{0}^{*} T X
$$

does not vanish over $\overline{\mathcal{M}}_{1,1} \times \overline{\mathfrak{M}}_{0,\{0\} \cup[k]}\left(X, A ; J, \nu_{B ; i} ; f\right)$. Thus, by Lemma 3.14 in $[\mathbf{Z 1}]$,

$$
\begin{array}{r}
N\left(\widetilde{\mathcal{D}}_{\mathcal{T}}\right)=\left\langle c\left(\pi_{P}^{*} \mathbb{E}^{*} \otimes \pi_{i}^{*} \operatorname{ev}_{0}^{*} T X\right) c\left(\pi_{P}^{*} L_{1} \otimes \pi_{i}^{*} \pi^{*} L_{0}\right)^{-1},\right. \\
\left.\overline{\mathcal{M}}_{1,1} \times \overline{\mathfrak{M}}_{0,\{0\} \cup[k]}\left(X, A ; J, \nu_{B ; i} ; f\right)\right\rangle \\
=-\frac{1}{24}\left\langle c_{1}(T X)+\pi^{*} c_{1}\left(L_{0}^{*}\right), \overline{\mathfrak{M}}_{0,\{0\} \cup[k]}\left(X, A ; J, \nu_{B ; i} ; f\right)\right\rangle .
\end{array}
$$

By the divisor and dilaton relations for GW-invariants,

$$
\begin{array}{r}
\left\langle c_{1}(T X), \overline{\mathfrak{M}}_{0,\{0\} \cup[k]}\left(X, A ; J, \nu_{B ; i} ; f\right)\right\rangle \\
\quad=\left\langle c_{1}(T X), A\right\rangle \cdot \mathrm{GW}_{0, k}^{X}(A ; \psi), \\
\left\langle\pi^{*} c_{1}\left(L_{0}^{*}\right), \overline{\mathfrak{M}}_{0,\{0\} \cup[k]}\left(X, A ; J, \nu_{B ; i} ; f\right)\right\rangle=-2 \cdot \mathrm{GW}_{0, k}^{X}(A ; \psi) ;
\end{array}
$$

see Section 26.3 in [MirSym], for example. Combining (3.23) with (3.24) and (3.25), we conclude that

$$
N\left(\widetilde{\mathcal{D}}_{\mathcal{T}}\right)=\frac{2-\left\langle c_{1}(T X), A\right\rangle}{24} \mathrm{GW}_{0, k}^{X}(A ; \psi) .
$$

The proof of Theorem 1.1 is now complete. 


\section{References}

[BCOV] M. Bershadsky, S. Cecotti, H. Ooguri \& C. Vafa, Holomorphic anomalies in topological field theories, Nucl. Phys. B405 (1993), 279-304, MR 1240687, Zbl 0919.58067.

[BeFa] K. Behrend \& B. Fantechi, The intrinsic normal cone, Invent. Math. 128 (1997), no. 1, 45-88, MR 1437495, Zbl 0909.14006.

[FlHS] A. Floer, H. Hofer \& D. Salamon, Transversality in elliptic Morse theory for the symplectic action, Duke Math. J. 80 (1996), no. 1, 251-292, MR 1360618, Zbl 0846.58025.

[FuOn] K. Fukaya \& K. Ono, Arnold Conjecture and Gromov-Witten invariant, Topology 38 (1999), no. 5, 933-1048, MR 1688434, Zbl 0946.53047.

[Ge] E. Getzler, The Elliptic Gromov-Witten invariants of $\mathbb{P}^{3}$, math.AG/ 9612009

[CDGP] P. Candelas, X. de la Ossa, P. Green \& L. Parkes, A pair of Calabi-Yau manifolds as an exactly soluble superconformal theory, Nuclear Phys. B359 (1991), 21-74, MR 1115626, Zbl 0904.32019.

[LT] J. Li \& G. Tian, Virtual moduli cycles and Gromov-Witten invariants of general symplectic manifolds, Topics in Symplectic 4-Manifolds, 4783, First Int. Press Lect. Ser., I, Internat. Press, 1998, MR 1635695, Zbl 0978.53136.

[LZ] J. Li \& A. Zinger, On the genus-one Gromov-Witten invariants of complete intersections, math.AG/0507104, to appear in JDG.

[McSa] D. McDuff \& D. Salamon, J-Holomorphic Curves and quantum cohomology, American Mathematical Society, 1994, MR 1286255, Zbl 0809.53002.

[MirSym] K. Hori, S. Katz, A. Klemm, R. Pandharipande, R. Thomas, C. Vafa, R. Vakil \& E. Zaslow, Mirror Symmetry, Clay Math. Inst., Amer. Math. Soc., August 2003, MR 2003030, Zbl 1044.14018.

[Pa] R. Pandharipande, Hodge integrals and degenerate contributions, Comm. Math. Phys. 208 (1999), no. 2, 489-506, MR 1729095, Zbl 0953.14036.

[RT] Y. Ruan \& G. Tian, A mathematical theory of quantum cohomology, J. Differential Geom. 42 (1995), no. 2, 259-367, MR 1366548, Zbl 0860.58005.

[VaZ1] R. Vakil \& A. Zinger, A desingularization of the main component of the moduli space of genus-one stable maps into $\mathbb{P}^{n}$, Geom.\&Top. 12 (2008) 1-95, MR 2377245, Zbl 1134.14009.

[VaZ2] R. Vakil \& A. Zinger, A natural smooth compactification of the space of elliptic curves in projective space, Elec. Res. Ann. Amer. Math. Soc. 13 (2007), 53-59, MR 2320682, Zbl 1131.14028.

[Z1] A. Zinger, Enumeration of genus-two curves with a fixed complex structure in $\mathbb{P}^{2}$ and $\mathbb{P}^{3}$, J. Differential Geom. 65 (2003), no. 3, 341-467, MR 2064428, Zbl 1070.14053.

[Z2] A. Zinger, Enumerative vs. symplectic invariants and obstruction bundles, J. Symplectic Geom. 2 (2004), no. 4, 445-543, MR 2197218, Zbl 1095.14051.

[Z3] A. Zinger, Enumeration of one-nodal rational curves in projective spaces, Topology 43 (2004), no. 4, pp 793-829, MR 2061208, Zbl 1060.53081. 
[Z4] A. Zinger, A sharp compactness theorem for genus-one pseudo-holomorphic maps, Geom.\&Top. 13 (2009) 2427-2522.

[Z5] A. Zinger, The reduced genus-one Gromov-Witten invariants of Calabi-Yau hypersurfaces, J. Amer. Math. Soc. 22 (2009), 691-737.

[Z6] A. Zinger, Standard vs. reduced genus-one Gromov-Witten invariants, Geom.\&Top. 12 (2008), 1203-1241, MR 2403808, Zbl pre05288419.

\section{Department of Mathematics STANFORD UNIVERSITY STANFORD, CA 94305-2125}

Current address:

Department of Mathematics

SUNY Stony Brook

Stony Brook, NY 11794-3651

E-mail address: azinger@math.sunysb.edu 